\title{
Uso de Métodos Bayesianos na Análise de Dados de Confiabilidade de Software Considerando Tempos entre Falhas
}

\author{
Daniela Brassolatti
}

Orientador: Prof. Dr. Jorge Alberto Achcar

esentada ao Instituto de Ciências Matemáticas de São omo parte dos requisitos necessários para obtenção do título ências - Área: Ciências de Computação e Matemática

$$
\text { USP }
$$

Maio - 1997 
O saber não tem limites, estamos sempre aprendendo...

Vivendo, aprendendo e aperfeiçoando...

Estamos todos funcionando numa fração pequena

de nossa capacidade de criar, aprender e aventurar-se...

Com vontade e coragem conquistamos o mundo.

Ao meu esposo Márcio,

Ao nosso Nêne, Víctor,

Aos meus pais

Ivo e Vírginia 


\section{Agradecimentos}

Em primeiro lugar, agradeço à Deus por me dar toda força para desenvolver este trabalho.

A conclusão dessa dissertação também não seria possível sem o auxílio de algumas pessoas e instituições que de alguma forma contribuiram para este projeto, as quais expresso meus sinceros agradecimentos.

Ao Prof. Dr. Jorge Alberto Achcar pela orientação segura e dedicada em todo o decorrer do projeto de pesquisa.

Ao Prof. Dr. Josemar Rodrigues pelo incentivo e apoio demonstrado durante as aulas e nos seminários informais.

Ao Prof. Dr. Luís Aparecido Milan e ao Prof. Dr. Josemar Rodrigues pelas valiosas sugestões quando da realização do meu exame de qualificação. questão.

Ao Prof. Dr. Marinho Gomes de Andrade pelas valiosas sugestões sobre o tema em

À CAPES pelo apoio financeiro.

Aos professores, funcionários, colegas de pós-graduação e a todos aqueles que de alguma maneira contribuiram para a realização deste trabalho. Em especial aos amigos Luciano, Daniele e Karin pela amizade, carinho e apoio durante este período, dentro e fora do ICMSC e à Karina pelo excelente trabalho de digitação dos manuscritos.

Especialmente ao meu esposo e ao meu filhinho pelo amor, paciência e incentivo. 


\section{Resumo}

Nesta dissertação de mestrado, apresentamos análises Clássica e Bayesiana para os principais modelos de Estratégia tipo I, estratégia de modelos de confiabilidade de software que modelam os tempos entre falhas do software. $\mathrm{Na}$ análise Clássica, estimadores pontuais e intervalos de confiança são encontrados usando métodos assintóticos. Na análise Bayesiana, considerando densidades a priori informativas para os parâmetros dos modelos, determinamos os resumos a posteriori, utilizando os métodos de simulação Gibbs Sampling e Metrópolis Hastings.

Em particular, consideramos diferentes densidades a priori para os parâmetros do modelo de Jelinski e Moranda (1972) (um dos primeiros modelos de confiabilidade de software desenvolvido) e verificamos a consequência de uma reparametrização para esse modelo. Também, apresentamos a técnica das distribuições preditivas condicionais ordenadas (CPO) para selecionar o melhor modelo dentre os modelos analisados.

Finalizamos, ilustrando os métodos propostos através de um exemplo prático. 


\section{Abstract}

In this dissertation we present Classical and Bayesian analyses for the most important models of strategy of type-I used in software reliability to model times between failures. In the Classical approach, point estimators and confidence intervals are obtained using assymptotical methods. In the Bayesian approach, considering informative prior densities for the parameters of the models, we obtain posterior summaries of interest, using the simulatim algorithms Gibbs Sampling and Metrópolis Hastings.

In special, we consider different prior densities for the parameters of the Jelinki and Moranda (1972) model and we also check the effects of a reparametrization in the obtained inferences. We also consider the technique of ordinated conditional predictive distributions (CPO) to select the best model among all considered models.

We conclude the work, presenting a practical example as a numerical illustration of the proposed methodology. 


\section{CONTEÚDO}

1 Introdução

1.1 Estratégias de Modelagem 2

1.1.1 A Estratégia tipo I__ 2

1.1.2 A Estratégia tipo II___ 3

1.2 Principais Modelos de Estratégia tipo I___ 3

1.3 Objetivos Principais da Pesquisa___ 6

2 Análise Clássica para Alguns Modelos de Estratégia tipo I

2.1 O Modelo de Jelinski e Moranda (1972)__ 8

2.1.1 Formulação do Modelo de Jelinski e Moranda___ 9

2.1.2 Estimadores de Máxima Verossimilhança para os parâmetros $\lambda$ e N

2.1.3 Inferências sobre os parâmetros $\lambda$ e $N$ 12

2.2 O Modelo de Moranda (1975) 14

2.2.1 Formulação do Modelo de Moranda 15 
2.2.2 Estimadores de Máxima Verossimilhança

para os parâmetros $\theta$ e K 16

2.2.3 Inferências sobre os parâmetros $\theta$ e K 17

2.3 O Modelo de Goel e Okumoto (1978) 19

2.3.1 Formulação do Modelo de Goel e Okumoto 20

2.3.2 Estimadores de Máxima Verossimilhança para os parâmetros

$\lambda, \mathrm{N}$ e $\mathrm{p}$ 21

2.3.3 Inferências sobre os parâmetros $\lambda, \mathrm{Ne} \mathrm{p}$ 22

2.4 O Modelo de Schick e Wolverton (1978) 25

2.4.1 Formulação do Modelo de Schick e Wolverton 26

2.4.2 Estimadores de Máxima Verossimilhança para os parâmetros $\lambda$ e $\mathrm{N}$ 27

2.4.3 Inferências sobre os parâmetros $\lambda$ e $\mathrm{N}$ 28

3 Uma Análise Bayesiana para os Principais

Modelos de Estratégia tipo I-1

3.1 Fórmula de Bayes 31

3.2 Densidades a Priori e a Posteriori 32

3.3 Uma Análise Bayesiana para o Modelo de Jelinski e Moranda 33

3.3.1 Densidades a Priori assumindo $\lambda$ com Distribuição Gama e $N$ com Distribuição Poisson 34

3.3.2 Densidades a Priori assumindo $\lambda$ com Distribuição Gama e $N$ com Distribuição Binomial Negativa 
3.3.3 Densidades a Priori assumindo $\lambda$ com Distribuição Gama e N com Distribuição Logarítmica

3.3.4 Uma Análise Bayesiana Hierárquica para

Modelo de Jelinski e Moranda 42

3.4 Uma Análise Bayesiana para o Modelo de Moranda 46

3.4.1 Densidades a Priori para $\theta$ e K 46

3.4.2 Densidade a Posteriori Conjunta para $\theta$ e K 47

3.4.3 Densidades a Posteriori Condicionais para $\theta$ e K 47

3.5 Uma Análise Bayesiana para o Modelo de Goel e Okumoto 48

3.5.1 Densidades a Priori para $\lambda, \mathrm{N}$ e $\mathrm{p}$ 49

3.5.2 Densidade a Posteriori Conjunta para $\lambda, \mathrm{N}$ e $\mathrm{p}$ 49

3.5.3 Densidades a Posteriori Condicionais para $\lambda, \mathrm{N}$ e $\mathrm{p}$

3.6 Uma Análise Bayesiana para o Modelo de Schick e Wolverton 50

3.6.1 Densidades a Priori para $\lambda$ e $\mathrm{N}$ 51

3.6.2 Densidade a Posteriori Conjunta para $\lambda$ e $N$ 51

3.6.3 Densidades a Posteriori Condicionais para $\lambda$ e $N$ 52

3.7 O Modelo de Littlewood e Verral 53

3.7.1 Formulação do Modelo de Littlewood e Verral 53

3.7.2 Uma Análise Bayesiana para o Modelo de Littlewood e Verral 54

3.7.2.1 Densidades a Priori para $\alpha, \beta_{0}$ e $\beta_{1}$ 55 
3.7.2.2 Densidade a Posteriori Conjunta para $\alpha, \beta_{0}$ e $\beta_{1}$ 55

3.7.2.3 Densidades a Posteriori Condicionais para $\alpha, \beta_{0}$ e $\beta_{1}$ 56

4 Algumas Considerações sobre Seleção de Modelos

4.1 Distribuições Preditivas 57

4.2 Estimativas de Monte Carlo para CPO's 60

4.3 Escolha de Modelos 61

5 Um Exemplo de Aplicação

5.1 Análise Clássica para os dados 64

5.1.1 Análise Clássica dos dados NTDS sob o Modelo de Jelinski e Moranda 64

5.1.1.1 O Efeito de uma Reparametrização para o Modelo de Jelinski e Moranda 65

5.1.1.2 Inferências para a Taxa de Falha considerando a Função de Verossimilhança "Profile" 66

5.1.1.3 T-plot de Hills e Smith 67

5.1.1.4 Terceira Derivada Padronizada do Logarítmo da Verossimilhança 68

5.1.2 Análise Clássica dos Dados NTDS sob o Modelo de Moranda 70

5.1.3 Análise Clássica dos Dados NTDS sob o Modelo de Schick e Wolverton 70 
5.2 Uma Solução Bayesiana na Análise dos Dados NTDS 71

5.2.1 Análise Bayesiana para os Dados NTDS sob o Modelo de Jelinski e Moranda 71

5.2.2 Análise Bayesiana para os Dados NTDS sob o Modelo de Moranda 86

5.2.3 Análise Bayesiana para os Dados NTDS sob o Modelo de Goel e Okumoto 89

5.2.4 Análise Bayesiana para os Dados NTDS sob o Modelo de Schick e Wolverton 92

5.2.5 Análise Bayesiana para os Dados NTDS sob o Modelo de Littlewood e Verral 95

5.3 Seleção de Modelos 99

6 Conclusões Finais e Perspectivas Futuras

Apêndice 1: O Algorítmo Gibbs Sampling 103

Apêndice 2: O Algorítmo Metrópolis Hastings 105

Apêndice 3: Verificação de Convergência 107

Apêndice 4: Alguns Programas Desenvolvidos 109

Bibliografia 


\section{Capítulo 1}

\section{INTRODUÇÃO}

A indústria relacionada à informática tem atualmente uma parcela significativa na economia mundial. Sua expansão segue com muita rapidez e desde que o software é a principal parte dos sistemas de muitos computadores, o campo de tecnologia de software pode esperar um crescimento similar.

O software é um sistema complexo de programas, rotinas e linguagens simbólicas que controlam o funcionamento do hardware (a parte física) de um computador e dirige sua operação.

Há duas características fundamentais na produção de um software que podem ser referidas como: qualidade e custo. Nesse ponto é que a confiabilidade se torna a característica mais importante inerente ao conceito "qualidade do software". Os acessos iniciais para medir a qualidade de um software baseiam-se no conceito de falha ou defeito encontrados num programa.

O programa tem que estar sendo executado para que uma falha ocorra. $\mathrm{O}$ termo falha se relaciona ao comportamento do programa. Ela não é a mesma coisa que defeito ou, mais apropriadamente, erro. Um erro é um defeito no programa que quando executado sob condições particulares, pode causar uma falha, isto é, uma falha é criada quando o programador comete um erro.

Nesse sentido, a confiabilidade de um software pode ser definida como sendo a probabilidade de que o software trabalha sem falha por um período de tempo especificado.

Para designar a confiabilidade de um software, a modelagem se torna um elemento essencial no sentido de tomar decisões em aplicações práticas.

Em geral, muitas pesquisas e modelos de confiabilidade de software foram desenvolvidos. Assim como conhecimentos práticos tem sido colhidos para determinar o melhor meio de se controlar o desenvolvimento de um software. 


\subsection{Estratégias de Modelagem}

Para modelar a confiabilidade de um software é necessário primeiramente considerar os principais fatores que a afetam. Esses fatores podem ser referidos como "introdução de erros" e "remoção de erros".

Os erros são introduzidos quando o software está sendo desenvolvido pelos programadores. Eles podem introduzir os erros durante o projeto original, ou quando estão acrescentando novos aspectos ou ainda quando estão consertando erros que tenham sido identificados. Uma vez que um erro é encontrado, ele pode ser corrigido (removido) e esperançosamente pode não originar mais falhas. A remoção de erros resultante da execução do programa depende da ocorrência da falha associada.

Na literatura, observamos que em confiabilidade de software existem duas estratégias de modelagem amplamente utilizadas. Essas estratégias cobrem a maioria dos modelos propostos e podem ser divididas em dois grupos, conhecidos como Estratégia tipo I e Estratégia tipo II.

\subsubsection{A Estratégia tipo I}

A Estratégia tipo I pode ser definida como uma estratégia de modelos que modelam os tempos entre falhas consecutivas do software. Essa estratégia pode ser dividida em dois sub-tipos.

O primeiro sub-tipo é onde o modelo se deriva da consideração da taxa de falha. A taxa de falha pode ser vista como o número de falhas por unidade de tempo, ou formalmente pode ser definida como a razão da função densidade de $\mathrm{T}$ por $\mathrm{P}(\mathrm{T} \geq \mathrm{t})$ se é sabido que está trabalhando no tempo $t$, onde $\mathrm{T}$ é uma variável aleatória que denota o tempo da primeira falha e $\mathrm{P}(\mathrm{T} \geq \mathrm{t})$ é a função de sobrevivência de $\mathrm{T}$.

Quando o modelo é definido através da taxa de falha nos tempos consecutivos entre falhas do software, a estratégia de modelagem é definida como estratégia tipo I-1.

Quando os erros são descobertos e removidos, a sequência da taxa de falha pode mostrar uma tendência decrescente e dessa forma a confiabilidade tende a aumentar. 
Um modo alternativo de modelar os tempos entre falhas, é definir uma relação estocástica entre tempos de sucessivas falhas. Esta estratégia será definida como estratégia tipo I-2.

A estratégia tipo I-2 modela diretamente os tempos entre falhas sem preocupar-se com as taxas de falha. Por exemplo, seja $T_{1}, \ldots, T_{i}$, variáveis aleatórias denotando os tempos entre sucessivas falhas do software. Num simples caso é possível declarar $T_{i+1}=\rho T_{i}+E_{i}$, onde $\rho \geq 0$ é uma constante e $\mathrm{E}_{\mathrm{i}}$ é um termo de distúrbio (tipicamente alguma variável aleatória com média zero). Então $\rho<1$ indicaria decréscimo de tempos entre falhas, ou seja, a confiabilidade esperada do software torna-se pior; $\rho=1$ indicaria nenhuma mudança esperada na confiabilidade do software e $\rho>1$ indicaria um acréscimo dos tempos entre falhas, ou seja, a confiabilidade do software melhora.

\subsubsection{A Estratégia tipo II}

A Estratégia tipo II é a estratégia de modelos que modelam o número de falhas do software para um dado tempo.O número de falhas do software $(\mathrm{M}(\mathrm{t}))$, observados durante o tempo $[0, t)$, pode ser modelado por um processo de Poisson com função de valor médio $\mu(t)$.

A função de valor médio representa as falhas médias cumulativas associadas com cada ponto do tempo.

$\mathrm{O}$ número de falhas do software pode também ser especificado por sua função de intensidade $\lambda(\mathrm{t})$. A intensidade de falha é um meio alternativo de se expressar a confiabilidade. Ela pode ser descrita como a taxa de mudança de uma função de valor médio, ou pode ser formalmente definida como a derivada da função de valor médio $\mu(\mathrm{t})$ com respeito a t. Se $\lambda(\mathrm{t})$ é uma constante $(\mu(\mathrm{t})$ é linear), então $\mathrm{M}(\mathrm{t})$ (o número de falhas) é chamado um processo Poisson homogêneo, em caso contrário é chamado processo de Poisson não-homogêneo.

\subsection{Principais Modelos de Estratégia tipo I.}

Muitos modelos para confiabilidade de software são propostos na literatura. Neste trabalho destacamos os mais populares modelos de estratégia tipo I, modelos que modelam os tempos entre falhas do software e os descrevemos brevemente abaixo. 


\section{O Modelo de Jelinski e Moranda (1972).}

Esse modelo, foi um dos primeiros modelos de confiabilidade de software. É um modelo referente a estratégia tipo I, que modela os tempos entre falhas do software considerando suas taxas de falha.

Jelinski e Moranda assumiram que todos os elementos que causam a falha estão associados num processo de Poisson homogêneo com taxa de falha $\lambda$.

Eles consideram o tempo $T_{i}(i=1,2, \ldots, N)$, entre a (i - 1)- ésima falha e a $\mathrm{i}$ - ésima falha, uma variável aleatória com distribuição Exponencial e a taxa de falha para o i - ésimo tempo entre falha $T_{i}$ é assumida ser uma constante proporcional ao número de erros restantes no programa ( $N$ - i + 1), ou seja, a taxa de falha para o modelo de Jelinski e Moranda, é dada por:

$$
\lambda_{\mathrm{i}}=\lambda(\mathrm{N}-\mathrm{i}+1), \mathrm{i}=1,2,3, \ldots, \mathrm{t} \geq 0 .
$$

\section{O Modelo de Moranda (1975).}

O modelo de Moranda é também um dos principais modelos de Estratégia tipo I que considera as taxas de falha para os tempos entre falhas.

Moranda supõe que a taxa de falha do software permanece constante para cada tempo entre falha $T_{i}$, mas após cada falha ela decresce geométricamente em i. Portanto, para constantes $\theta$ e K, a taxa de falha para o modelo de Moranda é dada por:

$$
\lambda_{\mathrm{i}}=\theta \mathrm{K}^{\mathrm{i}-1} .
$$




\section{O Modelo de Goel e Okumoto (1978).}

O modelo de Goel e Okumoto pode ser visto como uma tentativa de melhorar o modelo de Jelinski e Moranda (1972) pela alteração da suposição de que um reparo perfeito de um erro sempre ocorre.

Goel e Okumoto assumem que existe uma probabilidade $p, 0 \leq p \leq 1$, de corrigir um erro quando ele é encontrado. Portanto, a taxa de falha dos tempos entre falhas $\mathrm{T}_{\mathrm{i}}$, para esse modelo, é dada por:

$$
\lambda_{\mathrm{i}}=\lambda(\mathrm{N}-\mathrm{p}(\mathrm{i}-1))
$$

\section{O Modelo de Schick e Wolverton (1978).}

O modelo de Schick e Wolverton também faz uso da estratégia tipo I-1, que modela os tempos entre falhas consecutivas do software considerando suas taxas de falha.

Schick e Wolverton assumem que a taxa de falha é proporcional ao número de erros não corrigidos no programa e o tempo que foi decorrido desde a última falha. Portanto, a taxa de falha para o modelo de Schick e Wolverton é dada por:

$$
\lambda_{\mathrm{i}}=\lambda(\mathrm{N}-\mathrm{i}+1) \mathrm{t}, \mathrm{t} \geq 0 .
$$

\section{O Modelo de Littlewood e Verral (1973).}

O modelo de Littlewood e Verral é um modelo Bayesiano de estratégia tipo I, não desenvolvido pela caracterização da taxa de falha.

Littlewood e Verral assumem que o i-ésimo tempo entre falhas tem distribuição Exponencial com taxa de falha $\lambda_{i}$ e assumem uma distribuição a priori Gama para $\lambda_{i}$, com 
parâmetro $\alpha$ e escalar $\psi(i) . \psi(i)$ é uma função de i monotonicamente crescente, suposta para descrever a qualidade do programador e a tarefa do programa. Eles assumem $\psi(\mathrm{i})=\beta_{0}+\beta_{1} \mathrm{i}$ ( também considerado por Mazzuchi e Soyer, 1988).

Outros modelos são propostos na literatura usando estratégias de modelagem de tipo I-1 e I-2, porém, em muitas aplicações, as falhas do software são dadas em termos dos números de falhas que ocorrem em períodos fixos de tempo.

Quando isso ocorre, Moranda (1975) assume um processo de Poisson para descrever o número de falhas em cada período de tempo, fazendo uso da estratégia denominada estratégia tipo II.

Outros modelos envolvendo estratégia tipo II são propostos por Goel e Okumoto (1979), Goel (1983), Musa e Okumoto (1984), etc.

Todos esses modelos podem ser utilizados para medir a confiabilidade do software, analisar dados de falha, fazer inferências sobre o procedimento futuro do software e tomar decisões durante o processo do teste do software.

\subsection{Objetivos Principais da Pesquisa.}

O projeto de pesquisa apresentado neste trabalho tem como objetivo principal explorar o uso dos algorítmos de simulação via cadeias de Markov conhecidos como algorítmo Gibbs Sampling e algorítmo Metrópolis Hastings (ver por exemplo Casella e George, 1992; Gamerman, 1996; Apêndice 1 e 2), na análise dos mais populares modelos de Estratégia tipo I, estratégia de modelos que modelam os tempos entre falhas consecutivas do software, dando ênfase maior ao modelo de Jelinski e Moranda por ser um dos primeiros e mais usuais modelos de confiabilidade de software.

No Capítulo 2, apresentamos uma análise Clássica para os modelos de Jelinski e Moranda (1972), Moranda (1975), Schick e Wolverton (1978) e Goel e Okumoto (1978) considerando uma distribuição Exponencial para os tempos entre falhas do software.

Considerando densidades a priori informativas para os parâmetros envolvidos nos modelos de Jelinski e Moranda (1972), Moranda (1975), Goel e Okumoto (1978), Schick e Wolverton (1978) e Littlewood e Verral (1973), desenvolvemos no Capítulo 3, uma análise Bayesiana utilizando os algoritmos Gibbs Sampling e Metrópolis Hastings para os referidos modelos. 
A análise do modelo de Jelinski e Moranda (1972) é explorada considerando diferentes densidades a priori para os parâmetros de interesse e englobando densidades a priori hierárquicas.

Ainda considerando os principais modelos de Estratégia tipo I e uma distribuição Exponencial para os tempos entre falhas do software, desenvolvemos no Capítulo 4 um procedimento para selecionar modelos, utilizando densidades preditivas (ver por exemplo Gelfand, Dey e Chang; 1992).

Um exemplo de aplicação das metodologias descritas, é desenvolvido no Capítulo 5, considerando um conjunto de dados reais sobre tempos entre falhas do software.

Uma reparametrização para o modelo de Jelinski e Moranda, é adotada, com o intuito de avaliar as inferências obtidas considerando as aproximações normais usuais para os estimadores de máxima verossimilhança da taxa de falha $\lambda$.

Finalmente no Capítulo 6, são apresentadas algumas conclusões e comentários sobre as perspectivas futuras de pesquisa vinculadas a confiabilidade de software considerando os tempos entre falhas do software. 
Capítulo 2

\section{ANÁLISE CLÁSSICA PARA ALGUNS MODELOS DE ESTRATÉGIA \\ TIPO I}

O modelo de Jelinski e Moranda (1972), encontra-se entre os principais modelos que descrevem os tempos entre falhas do software. Algumas modificações desse modelo são introduzidas na literatura por Moranda (1975), Goel e Okumoto (1978), Schick e Wolverton (1978) e Littlewood e Verral (1973).

Neste capítulo será apresentada uma análise clássica para determinação de estimadores de máxima verossimilhança e intervalos de confiança para os parâmetros envolvidos nos principais modelos referentes a estratégia de tipo I-1 (estratégia de modelos que modelam os tempos entre falhas do software), considerando a distribuição Exponencial (amplamente utilizada em confiabilidade) para expressar matematicamente o comportamento dos tempos entre falhas do software.

\subsection{O Modelo de Jelinski e Moranda (1972)}

O modelo de Jelinski e Moranda (JM) formou alicerce para muitos modelos desenvolvidos posteriormente. Esse modelo se caracteriza pela modelagem dos tempos entre falhas do software considerando sua taxa de falha, sendo um dos principais modelos referentes à estratégia de tipo I1 .

Além disso, apesar de intuitivamente observarmos que a taxa de falha cresce com o uso (envelhecimento) para a maioria dos componentes manufaturados, Jelinski e Moranda assumem que a cada falha do software, um reparo perfeito é executado não criando novos erros, fazendo assim, que a taxa de falha do software decresça a cada reparo (veja figura 2.1 que mostra a taxa de falha para o modelo de Jelinski e Moranda). 


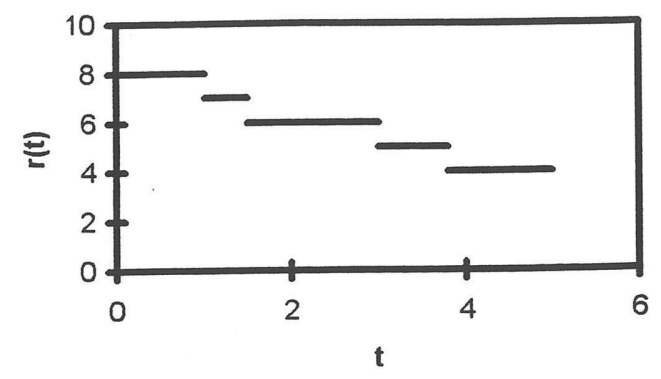

Figura 2.1: Taxa de falha para o modelo JM

\subsubsection{Formulação do Modelo de Jelinski e Moranda}

A modelagem descrita nesta seção será definida a partir da taxa de falha do software e a distribuição Exponencial para os tempos entre falhas do software.

Supondo que o número de erros no software seja N, assume-se que a taxa de falha para o i-ésimo tempo entre falhas $\mathrm{T}_{\mathrm{i}}$ é uma constante proporcional à $(\mathrm{N}-\mathrm{i}+1)$, correspondente ao número de erros restantes no software, ou seja,

$$
\lambda_{\mathrm{i}}=\lambda(\mathrm{N}-\mathrm{i}+1), \mathrm{i}=1,2, \ldots, \mathrm{t} \geq 0 \text { para alguma constante } \lambda .
$$

Seja $T_{i}$ uma variável aleatória não-negativa denotando os tempos entre falhas do software com uma distribuição Exponencial com função densidade de probabilidade (f.d.p) dada por,

$$
\mathrm{f}\left(\mathrm{t}_{\mathrm{i}} \mid \lambda_{\mathrm{i}}\right)=\lambda_{\mathrm{i}} \mathrm{e}^{-\lambda_{\mathrm{i}} \mathrm{t}_{\mathrm{i}}}
$$

onde $\lambda_{\mathrm{i}}=\lambda(\mathrm{N}-\mathrm{i}+1)$ é um parâmetro desconhecido representando a taxa de falha no i-ésimo tempo entre falhas, $\mathrm{i}=1,2, \ldots, \mathrm{t} \geq 0$.

O tempo médio entre falhas, obtido calculando-se a esperança dos tempos entre falhas do software, é dado por, 


$$
E\left(T_{i}\right)=\frac{1}{\lambda(N-i+1)}
$$

Considerando $T_{1}, T_{2}, \ldots, T_{n}$ variáveis aleatórias independentes que denotam os tempos entre falhas do software, a função de verossimilhança para $\mathrm{N}$ e $\lambda$ é dada por,

$$
\mathrm{L}(\lambda, \mathrm{N})=\prod_{\mathrm{i}=1}^{\mathrm{n}} \mathrm{f}\left(\mathrm{t}_{\mathrm{i}} \mid \lambda_{\mathrm{i}}\right)
$$

onde $\lambda_{\mathrm{i}}=\lambda(\mathrm{N}-\mathrm{i}+1)$ e $\mathrm{f}\left(\mathrm{t}_{\mathrm{i}} / \lambda_{\mathrm{i}}\right)$ é a f.d.p (2.2).

Desenvolvendo (2.4) obtemos,

$$
L(\lambda, N)=\left[\prod_{i=1}^{n} \lambda_{i} e^{-\lambda_{i} t_{i}}\right]=\lambda^{n} \prod_{i=1}^{n}(N-i+1) \exp \left\{-\lambda\left[\sum_{i=1}^{n}(N-i+1) t_{i}\right]\right\} .
$$

Definindo $A(N)=\prod_{i=1}^{n}(N-i+1)$ e $B(N)=\sum_{i=1}^{n}(N-i+1) t_{i}$, temos:

$$
L(\lambda, N)=\lambda^{n} A(N) \exp \{-B(N) \lambda\}
$$

\subsubsection{Estimadores de Máxima Verossimilhança para os Parâmetros $\mathrm{N}$ e $\lambda$}

A partir do momento em que observamos os dados (no nosso caso, os tempos entre falhas do software), toda informação sobre os parâmetros ficará contida na função log-verossimilhança.

O logarítmo da função de verossimilhança para $\mathrm{N}$ e $\lambda$ é dado por, 


$$
1(\lambda, N)=n \log \lambda+\sum_{i=1}^{n} \ln (N-i+1)-\lambda \sum_{i=1}^{n}(N-i+1) t_{i}
$$

Assumindo N contínuo (isso é considerado por Singpurwalla e Wilson, 1994), podemos encontrar os estimadores de máxima verossimilhança para $\mathrm{N}$ e $\lambda$, derivando o logarítmo da verossimilhança (2.6).

As primeiras derivadas de $1(\lambda, N)$ com respeito a $\lambda$ e $N$ são dadas por,

$$
\begin{gathered}
\frac{\partial \mathrm{l}(\lambda, N)}{\partial \lambda}=\frac{\mathrm{n}}{\lambda}-\sum_{\mathrm{i}=1}^{\mathrm{n}}(\mathrm{N}-\mathrm{i}+1) \mathrm{t}_{\mathrm{i}}, \\
\frac{\partial \mathrm{l}(\lambda, \mathrm{N})}{\partial \mathrm{N}}=\sum_{\mathrm{i}=1}^{\mathrm{n}}\left(\frac{1}{\mathrm{~N}-\mathrm{i}+1}\right)-\lambda \sum_{\mathrm{i}=1}^{\mathrm{n}} \mathrm{t}_{\mathrm{i}} .
\end{gathered}
$$

Igualando a zero as primeiras derivadas (2.7) e (2.8), e resolvendo o sistema de equações em $\lambda$ e $\mathrm{N}$, obtemos os estimadores de máxima verossimilhança de $\lambda$ e $\mathrm{N}$ dados por:

Definindo $S_{n}=\sum_{i=1}^{n} t_{i}$

$$
\hat{\lambda}=\frac{\mathrm{n}}{\mathrm{NS}_{\mathrm{n}}-\sum_{\mathrm{i}=1}^{\mathrm{n}}(\mathrm{i}-1) \mathrm{t}_{\mathrm{i}}},
$$

e N̂́ a solução da equação,

$$
\sum_{i=1}^{n}\left(\frac{1}{N-i+1}\right)=\frac{n}{N-\frac{1}{S_{n}} \sum_{i=1}^{n}(i-1) t_{i}}
$$


As equações de verossimilhança (2.9) e (2.10) são não lineares. Para solucioná-las numéricamente, pode-se recorrer a métodos iterativos, como por exemplo o método de Newton Raphson. Alguns artigos foram escritos com o propósito de analisar técnicas numéricas para soluções de equações de verossimilhança, (ver por exemplo Zacks, 1971).

\subsubsection{Inferências Sobre os Parâmetros $\lambda$ e N}

Para inferências sobre os parâmetros de um determinado modelo, usualmente, utiliza-se a aproximação Normal assintótica dos estimadores de máxima verossimilhança, (ver por exemplo, Mood, Graybill e Boes, 1974). Assim consideramos,

$$
(\hat{\lambda}, \hat{N}) \stackrel{a}{\sim} \mathrm{N}\left\{(\lambda, N) ; I^{-1}(\hat{\lambda}, \hat{N})\right\}
$$

onde $\mathrm{I}(\lambda, N)$ é a matriz de informação de Fisher dada pelas esperanças das segundas derivadas parciais de $1(\lambda, N)$ com respeito a $\lambda$ e $\mathrm{N}$ a menos do sinal, (ver por exemplo, Box e Tiao, 1973).

As segundas derivadas parciais da função log-verossimilhança $1(\lambda, N)(2.5)$ com respeito a $\lambda$ e N são dadas por,

$$
\begin{gathered}
\frac{\partial^{2} 1(\lambda, N)}{\partial \lambda^{2}}=-\frac{n}{\lambda^{2}}, \\
\frac{\partial^{2} l(\lambda, N)}{\partial \lambda \partial N}=-\sum_{i=1}^{n} t_{i}, \\
\frac{\partial^{2} l(\lambda, N)}{\partial N^{2}}=\sum_{i=1}^{n}\left[-\frac{1}{(N-i+1)^{2}}\right] .
\end{gathered}
$$


Como já visto em (2.3), $\mathrm{E}\left(\mathrm{T}_{\mathrm{i}}\right)=\frac{1}{\lambda(\mathrm{N}-\mathrm{i}+1)}$, assim, a partir das segundas derivadas da função log-verossimilhança, tem-se que:

$$
\begin{gathered}
E\left\{\frac{-\partial^{2} 1(\lambda, N)}{\partial \lambda^{2}}\right\}=\frac{n}{\lambda^{2}} \\
E\left\{\frac{-\partial^{2} 1(\lambda, N)}{\partial \lambda \partial N}\right\}=\frac{1}{\lambda} \sum_{i=1}^{n}\left[\frac{1}{N-i+1}\right] \\
E\left\{\frac{-\partial^{2} 1(\lambda, N)}{\partial N^{2}}\right\}=\sum_{i=1}^{n}\left[\frac{1}{(N-i+1)^{2}}\right] .
\end{gathered}
$$

Portanto a matriz de Informação de Fisher para $\lambda$ e $\mathrm{N}$ é dada por,

$$
\mathrm{I}(\lambda, N)=\left[\begin{array}{cc}
\frac{\mathrm{n}}{\lambda^{2}} & \frac{1}{\lambda} \sum_{\mathrm{i}=1}^{\mathrm{n}}\left[\frac{1}{\mathrm{~N}-\mathrm{i}+1}\right] \\
\frac{1}{\lambda} \sum_{\mathrm{i}=1}^{\mathrm{n}}\left[\frac{1}{\mathrm{~N}-\mathrm{i}+1}\right] & \sum_{\mathrm{i}=1}^{\mathrm{n}}\left[\frac{1}{(\mathrm{~N}-\mathrm{i}+1)^{2}}\right]
\end{array}\right] .
$$

Note que $\mathrm{I}^{-1}(\hat{\Lambda}, \hat{\mathrm{N}})$, o inverso da matriz de Informação de Fisher, é a matriz de variâncias e covariâncias assintótica de $\hat{\lambda}$ e $\hat{N}$, dada por,

$$
\mathrm{I}^{-1}(\hat{\lambda}, \hat{\mathrm{N}})=\left[\begin{array}{cc}
\hat{\sigma}_{\lambda}^{2} & \hat{\sigma}_{\lambda N} \\
\hat{\sigma}_{\lambda N} & \hat{\sigma}_{N}^{2}
\end{array}\right]
$$

(ver por exemplo, Bickel \& Doksum, 1977). 
Considerando a distribuição Normal Assintótica (2.11) para os estimadores de máxima verossimilhança $\hat{\lambda}$ e $\hat{\mathrm{N}}$, é possível construir intervalos de confiança para os parâmetros $\lambda$ e $\mathrm{N}$.

Os intervalos de confiança para $\lambda$ e $N$, considerando um nível de confiança $100(1-\gamma) \%$, são dados por,

$$
\begin{aligned}
& \operatorname{ic}(\lambda)=\hat{\lambda}-Z_{\gamma / 2} \hat{\sigma}_{\lambda} ; \hat{\lambda}+Z_{\gamma / 2} \hat{\sigma}_{\lambda}, \\
& \operatorname{ic}(N)=\hat{N}-Z_{\gamma / 2} \hat{\sigma}_{N} ; \hat{N}+Z_{\gamma / 2} \hat{\sigma}_{N},
\end{aligned}
$$

onde $Z_{\gamma / 2}$ é um percentil da distribuição Normal padronizada.

\subsection{O Modelo de Moranda (1975)}

O modelo de Moranda (MO) (1975), é uma extensão do modelo de Jelinski e Moranda(1972), sendo também um modelo referente a estratégia tipo I-1, que modela os tempos entre falhas do software. Moranda supõe que fixando erros que causam as falhas antecipadas no sistema, reduzirá mais a taxa de falha, do que fixando erros que ocorrerão mais tarde, isso porque os erros antecipados (mais cedo) são mais prováveis serem os maiores. A suposição de um reparo perfeito, com nenhuma introdução de novos erros durante o reparo, é conservada.

Comparando com o modelo de JM (1972), onde a queda na taxa de falha após cada falha é $\lambda$, a queda na taxa de falha após a i-ésima falha para o modelo de Moranda é, $\theta(1-\mathrm{K}) \mathrm{K}^{\mathrm{i}-1}$ (veja figura 2.2 que mostra a taxa de falha para o modelo de Moranda). 


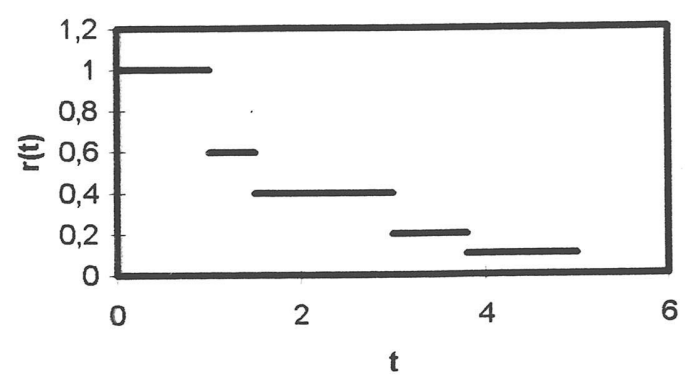

Figura 2.2: Taxa de falha para o modelo MO

\subsubsection{Formulação do Modelo de Moranda}

Para formular o modelo, Moranda (1975) propõe que a taxa de falha permanece constante para cada $T_{i}$, decrescendo geométricamente em i após cada falha, isto é, para constantes $\theta$ e $\mathrm{K}$, a taxa de falha para o modelo de Moranda é dada por,

$$
\lambda_{\mathrm{i}}=\theta \mathrm{K}^{\mathrm{i}-1}
$$

Sendo $T_{i}$ uma variável aleatória não-negativa independente, que denota o tempo entre falhas do software com uma distribuição Exponencial com função densidade de probabilidade (2.2), a função de verossimilhança para $\theta$ e K é dada por,

$$
L(\theta, K)=\prod_{i=1}^{n} f\left(t_{i} \mid \lambda_{i}\right)
$$

onde $\lambda_{\mathrm{i}}=\theta \mathrm{K}^{\mathrm{i}-1}$ representa a taxa de falha no i-ésimo tempo entre falhas, $\mathrm{i}=1,2, \ldots, \mathrm{t} \geq 0$.

Desenvolvendo (2.23) obtemos, 


$$
L(\theta, K)=\theta^{n} K^{\frac{n(n-1)}{2}} \exp \left\{-\theta \sum_{i=1}^{n} K^{i-1} t_{i}\right\} .
$$

\subsubsection{Estimadores de Máxima Verossimilhança para os Parâmetros $\theta$ e K}

Os estimadores de Máxima Verossimilhança para $\theta$ e $\mathrm{K}$ podem ser obtidos através do logarítmo da função de Verossimilhança (2.24).

O logarítmo dessa função é dado por,

$$
1(\theta, K)=n \ln \theta+\frac{n(n-1)}{2} \ln K-\theta \sum_{i=1}^{n} K^{i-1} t_{i}
$$

Os estimadores de máxima verossimilhança para $\theta$ e $\mathrm{K}$ são encontrados derivando o logarítmo da verossimilhança (2.25).

As primeiras derivadas de $1(\theta, K)$ com respeito a $\theta$ e $K$ são dadas por,

$$
\begin{gathered}
\frac{\partial \mathrm{l}(\theta, \mathrm{K})}{\partial \theta}=\frac{\mathrm{n}}{\theta}-\sum_{\mathrm{i}=1}^{\mathrm{n}} \mathrm{K}^{\mathrm{i}-1} \mathrm{t}_{\mathrm{i}}, \\
\frac{\partial \mathrm{l}(\theta, \mathrm{K})}{\partial \mathrm{K}}=\frac{\mathrm{n}(\mathrm{n}-1)}{2 \mathrm{~K}}-\theta \sum_{\mathrm{i}=1}^{\mathrm{n}}(\mathrm{i}-1) \mathrm{K}^{\mathrm{i}-2} \mathrm{t}_{\mathrm{i}} .
\end{gathered}
$$

Igualando a zero as primeiras derivadas (2.26) e (2.27), e resolvendo o sistema de equações em $\theta$ e $\mathrm{K}$, obtemos os estimadores de máxima verossimilhança de $\theta$ e $\mathrm{K}$, dados por, 


$$
\hat{\theta}=\frac{\mathrm{n}}{\sum_{\mathrm{i}=1}^{\mathrm{n}} \hat{\mathrm{K}}^{\mathrm{i}-1} \mathrm{t}_{\mathrm{i}}},
$$

e $\hat{\mathrm{K}}$ é a solução da equação,

$$
\frac{n \sum_{i=1}^{n}(i-1) K^{i-2} t_{i}}{\sum_{i=1}^{n} K^{i-1} t_{i}}=\frac{n(n-1)}{2 K} .
$$

Como as equações (2.28) e (2.29) são não lineares, suas soluções podem ser obtidas numéricamente com o auxílio de métodos iterativos, como por exemplo o método de Newton Raphson (ver por exemplo, Zacks, 1971).

\subsubsection{Inferências Sobre os Parâmetros $\theta$ e K}

Para fazermos inferências sobre os parâmetros $\theta$ e K, utilizamos a aproximação Normal assintótica dos estimadores de máxima verossimilhança, como já mencionado na seção (2.1.3), (ver por exemplo, Mood, Graybill e Boes, 1974).

Assim consideramos,

$$
(\hat{\theta}, \hat{\mathrm{K}}) \stackrel{\mathrm{a}}{\sim} \mathrm{N}\left\{(\theta, \mathrm{K}) ; \mathrm{I}^{-1}(\hat{\theta}, \hat{\mathrm{K}})\right\}
$$

onde $\mathrm{I}(\theta, \mathrm{K})$ é a matriz de informação de Fisher dada pelas esperanças das segundas derivadas parciais de $1(\theta, \mathrm{K})$ com respeito a $\theta$ e $\mathrm{K}$ a menos do sinal (ver por exemplo, Box e Tiao, 1973).

As segundas derivadas parciais da função log-verossimilhança $1(\theta, K)$ com respeito a $\theta$ e $\mathrm{K}$, são dadas por, 


$$
\begin{gathered}
\frac{\partial^{2} 1(\theta, K)}{\partial \theta^{2}}=-\frac{n}{\theta^{2}}, \\
\frac{\partial^{2} 1(\theta, K)}{\partial \theta \partial K}=-\sum_{i=1}^{n}(i-1) K^{i-2} t_{i}, \\
\frac{\partial^{2} l(\theta, K)}{\partial K^{2}}=-\frac{n(n-1)}{2 K^{2}}-\theta \sum_{i=1}^{n}(i-1)(i-2) K^{i-3} t_{i} .
\end{gathered}
$$

Sabendo que $E\left(T_{i}\right)=\frac{1}{\theta K^{i-1}}$, a partir das segundas derivadas da função logverossimilhança, tem-se que:

$$
\begin{gathered}
E\left\{\frac{-\partial^{2} 1(\theta, K)}{\partial \theta^{2}}\right\}=\frac{n}{\theta^{2}}, \\
E\left\{\frac{-\partial^{2} 1(\theta, K)}{\partial \theta \partial K}\right\}=\frac{1}{\theta K} \sum_{i=1}^{n}(i-1), \\
E\left\{\frac{-\partial^{2} 1(\theta, K)}{\partial K^{2}}\right\}=\frac{n(n-1)+2 \sum_{i=1}^{n}(i-1)(i-2)}{2 K^{2}} .
\end{gathered}
$$

Portanto a matriz de Informação de Fisher para $\theta$ e K é dada por,

$$
I(\theta, K)=\left[\begin{array}{cc}
\frac{n}{\theta^{2}} & \frac{1}{\theta K} \sum_{i=1}^{n}(i-1) \\
\frac{1}{\theta K} \sum_{i=1}^{n}(i-1) & \frac{n(n-1)+2 \sum_{i=1}^{n}(i-1)(i-2)}{2 K^{2}}
\end{array}\right] .
$$


$\mathrm{I}^{-1}(\hat{\theta}, \hat{\mathrm{K}})$, o inverso da matriz de Informação de Fisher, é a matriz de variâncias e covariâncias de $\hat{\theta}$ e $\hat{K}$, dada por,

$$
I^{-1}(\hat{\theta}, \hat{K})=\left[\begin{array}{ll}
\hat{\sigma}_{\theta}^{2} & \hat{\sigma}_{\theta K} \\
\hat{\sigma}_{\theta K} & \hat{\sigma}_{\mathrm{K}}^{2}
\end{array}\right],
$$

(ver por exemplo, Bickel \& Doksum, 1977).

Considerando a distribuição Normal Assintótica (2.30) para os estimadores de máxima verossimilhança $\hat{\theta}$ e $\hat{\mathrm{K}}$, podemos construir intervalos de confiança para $\theta$ e $\mathrm{K}$, considerando um nível de confiança $100(1-\gamma) \%$, dados por,.

$$
\begin{aligned}
& \operatorname{ic}(\theta)=\hat{\theta}-Z_{\gamma / 2} \hat{\sigma}_{\theta} ; \hat{\theta}+Z_{\gamma / 2} \hat{\sigma}_{\theta}, \\
& \operatorname{ic}(K)=\hat{K}-Z_{\gamma / 2} \hat{\sigma}_{K} ; \hat{K}+Z_{\gamma / 2} \hat{\sigma}_{K},
\end{aligned}
$$

onde $Z_{\gamma / 2}$ é um percentil da distribuição Normal padronizada.

\subsection{O Modelo de Goel e Okumoto (1978)}

Goel e Okumoto (GO) (1978) propuseram um modelo similar ao modelo de JM (1972), mas assumiram que existe uma probabilidade $p, 0 \leq p \leq 1$, de corrigir um erro quando ele é encontrado, o que significa que após i defeitos encontrados, espera-se (i x p) defeitos corrigidos, ao invés de $\mathrm{i}$. 


\subsubsection{Formulação do Modelo de Goel e Okumoto}

O modelo de Goel e Okumoto é formulado similarmente ao modelo de Jelinski e Moranda (1972). Considerando uma probabilidade p de corrigir o erro quando este é encontrado, a taxa de falha pode ser definida por:

$$
\lambda_{\mathrm{i}}=\lambda[\mathrm{N}-\mathrm{p}(\mathrm{i}-1)]
$$

Podemos notar que quando $\mathrm{p}=1$, temos o modelo de JM (2.1), ou seja, Jelinski e Moranda assumem que durante o reparo não há introdução de novos erros.

Considerando $T_{1}, \ldots, T_{n}$ variáveis aleatórias independentes que denotam os tempos entre falhas do software, com distribuição Exponencial com função densidade de probabilidade (2.2), a função de verossimilhança para $\lambda, \mathrm{N}$ e p é dada por,

$$
\mathrm{L}(\lambda, N, p)=\prod_{i=1}^{n} f\left(t_{i} \mid \lambda_{i}\right)=\prod_{i=1}^{n}\left\{\lambda[N-p(i-1)] \exp \left[-\lambda(N-p(i-1)) t_{i}\right]\right\}
$$

onde $\lambda_{i}=\lambda[N-p(i-1)]$ representa a taxa de falha $(2.41), i=1,2, \ldots, t \geq 0$.

Desenvolvendo (2.42), obtemos:

$$
L(\lambda, N, p)=\lambda^{n} \prod_{i=1}^{n}[N-p(i-1)] \exp \left\{-\lambda \sum_{i=1}^{n}[N-p(i-1)] t_{i}\right\} .
$$

Definindo $A(N, p)=\prod_{i=1}^{n}[N-p(i-1)]$ e $B(N, p)=\sum_{i=1}^{n}[N-p(i-1)] t_{i}$, temos,

$$
L(\lambda, N, p)=\lambda^{n} A(N, p) \exp \{-\lambda B(N, p)\}
$$




\subsubsection{Estimadores de Máxima Verossimilhança para os Parâmetros $\lambda, \mathbf{N}$ e p}

Os estimadores de máxima verossimilhança para $\lambda, \mathrm{N}$ e $\mathrm{p}$ são obtidos através do logarítmo da função de verossimilhança (2.43).

O logarítmo da função (2.43) é dado por,

$$
1(\lambda, N, p)=n \ln \lambda+\sum_{i=1}^{n} \ln [N-p(i-1)]-\lambda\left\{\sum_{i=1}^{n}[N-p(i-1)] t_{i}\right\} .
$$

Os estimadores de máxima verossimilhanças para $\lambda, \mathrm{N}$ e p são encontrados através das derivadas do logarítmo da verossimilhança (2.44).

As primeiras derivadas de $1(\lambda, N, p)$ com respeito a $\lambda, N$ e $p$ são dadas respectivamente por,

$$
\begin{gathered}
\frac{\partial l(\lambda, N, p)}{\partial \lambda}=\frac{n}{\lambda}-\sum_{i=1}^{n}[N-p(i-1)] t_{i}, \\
\frac{\partial l(\lambda, N, p)}{\partial N}=\sum_{i=1}^{n}\left(\frac{1}{N-p(i-1)}\right)-\lambda \sum_{i=1}^{n} t_{i}, \\
\frac{\partial l(\lambda, N, p)}{\partial p}=\sum_{i=1}^{n}\left(\frac{-(i-1)}{N-p(i-1)}\right)+\lambda \sum_{i=1}^{n}(i-1) t_{i} .
\end{gathered}
$$

Igualando a zero a expressão (2.45), obtemos o estimador de máxima verossimilhança para $\lambda$, dado pela solução da equação: 


$$
\hat{\lambda}=\frac{\mathrm{n}}{\sum_{\mathrm{i}=1}^{\mathrm{n}}[\hat{\mathrm{N}}-\hat{\mathrm{p}}(\mathrm{i}-1)] \mathrm{t}_{\mathrm{i}}},
$$

$\hat{\mathrm{N}}$ e $\hat{\mathrm{p}}$ podem ser obtidos através da solução das equações (2.49) e (2.50) abaixo, utilizando o método iterativo de Newton, (ver por exemplo Bazaraa e Shetty, 1979).

$$
\sum_{i=1}^{n}\left(\frac{1}{[\hat{N}-\hat{p}(i-1)]}\right)=\frac{n}{\hat{N}-\frac{\hat{p}}{S_{n}} \sum_{i=1}^{n}(i-1) t_{i}}
$$

onde $\mathrm{S}_{\mathrm{n}}=\sum_{\mathrm{i}=1}^{\mathrm{n}} \mathrm{t}_{\mathrm{i}}$

$$
\frac{n}{\left(\frac{N}{S_{p}} \sum_{i=1}^{n} t_{i}\right)-p}=\sum_{i=1}^{n}\left(\frac{(i-1)}{[N-p(i-1)]}\right)
$$

onde $S_{p}=\sum_{i=1}^{n}(i-1) t_{i}$

\subsubsection{Inferências Sobre os Parâmetros $\lambda, \mathbf{N}$ e p}

Utilizando a aproximação Normal assintótica dos estimadores de máxima verossimilhança, consideramos:

$$
(\hat{\lambda}, \hat{N}, \hat{p}) \stackrel{a}{\sim} N\left\{(\lambda, N, p) ; I^{-1}(\hat{\lambda}, \hat{N}, \hat{p})\right\},
$$

onde $\mathrm{I}(\lambda, \mathrm{N}, \mathrm{p})$ é a matriz de informação de Fisher dada pelas esperanças das segundas derivadas parciais de $1(\lambda, N, p)$ com respeito a $\lambda, N$ e $p$ a menos do sinal. 
As segundas derivadas da função log-verossimilhança (2.44) com respeito a $\lambda, \mathrm{N}$ e p são dadas por,

$$
\begin{gathered}
\frac{\partial^{2} 1(\lambda, N, p)}{\partial \lambda^{2}}=-\frac{n}{\lambda^{2}}, \\
\frac{\partial^{2} l(\lambda, N, p)}{\partial \lambda \partial N}=-\sum_{i=1}^{n} t_{i} \\
\frac{\partial^{2} l(\lambda, N, p)}{\partial \lambda \partial p}=\sum_{i=1}^{n}(i-1) t_{i} \\
\frac{\partial^{2} l(\lambda, N, p)}{\partial N N^{2}}=\sum_{i=1}^{n}\left[-\frac{1}{[N-p(i-1)]^{2}}\right] \\
\frac{\partial^{2} l(\lambda, N, p)}{\partial N \partial p}=\sum_{i=1}^{n}\left[-\frac{(-i+1)}{[N-p(i-1)]^{2}}\right] \\
\frac{\partial^{2} l(\lambda, N, p)}{\partial p^{2}}=\sum_{i=1}^{n}\left[-\frac{(i-1)^{2}}{[N-p(i-1)]^{2}}\right]
\end{gathered}
$$

Sabendo que $E\left(T_{i}\right)=\frac{1}{\lambda[N-p(i-1)]}$, a partir das segundas derivadas da função (2.44), obtemos:

$$
\mathrm{E}\left\{\frac{-\partial^{2} 1(\lambda, N, p)}{\partial \lambda^{2}}\right\}=\frac{n}{\lambda^{2}},
$$




$$
\begin{aligned}
& E\left\{\frac{-\partial^{2} 1(\lambda, N, p)}{\left.\partial N^{2}\right\}}\right\} \sum_{i=1}^{n}\left[\frac{1}{[N-p(i-1)]^{2}}\right], \\
& E\left\{\frac{-\partial^{2} 1(\lambda, N, p)}{\partial p^{2}}\right\}=\sum_{i=1}^{n}\left[\frac{(i-1)^{2}}{[N-p(i-1)]^{2}}\right], \\
& E\left\{\frac{-\partial^{2} 1(\lambda, N, p)}{\partial \lambda \partial N}\right\}=\frac{1}{\lambda} \sum_{i=1}^{n}\left[\frac{1}{[N-p(i-1)]}\right], \\
& E\left\{\frac{-\partial^{2} 1(\lambda, N, p)}{\partial \lambda \partial p}\right\}=-\frac{1}{\lambda} \sum_{i=1}^{n}\left[\frac{(i-1)}{[N-p(i-1)]}\right], \\
& E\left\{\frac{-\partial^{2} 1(\lambda, N, p)}{\partial N \partial p}\right\}=\sum_{i=1}^{n}\left[\frac{(-i+1)}{[N-p(i-1)]^{2}}\right] .
\end{aligned}
$$

Assim, a matriz de Informação de Fisher para $\lambda, \mathrm{N}$ e p é dada por,

$$
\mathrm{I}(\lambda, N, p)=\left[\begin{array}{lll}
a_{1} & a_{2} & a_{3} \\
a_{2} & a_{4} & a_{5} \\
a_{3} & a_{5} & a_{6}
\end{array}\right],
$$

onde $a_{1}=\frac{n}{\lambda^{2}}, a_{2}=\frac{1}{\lambda} \sum_{i=1}^{n} \frac{1}{[N-p(i-1)]}, a_{3}=\frac{1}{\lambda} \sum_{i=1}^{n}\left[\frac{(-i+1)}{[N-p(i-1)]}\right]$,

$$
a_{4}=\sum_{i=1}^{n} \frac{1}{[N-p(i-1)]^{2}}, a_{5}=\sum_{i=1}^{n}\left[\frac{(-i+1)}{[N-p(i-1)]^{2}}\right] \text { e } a_{6}=\sum_{i=1}^{n}\left[\frac{(i-1)^{2}}{[N-p(i-1)]^{2}}\right] \text {. }
$$


$\mathrm{I}^{-1}(\hat{\lambda}, \hat{\mathrm{N}}, \hat{\mathrm{p}})$, o inverso da matriz de Informação de Fisher, é a matriz de variâncias e covariâncias de $\hat{\lambda}, \hat{\mathrm{N}}$ e $\hat{\mathrm{p}}$, dada por,

$$
\mathrm{I}^{-1}(\hat{\lambda}, \hat{\mathrm{N}}, \hat{\mathrm{p}})=\left[\begin{array}{ccc}
\sigma_{\lambda}^{2} & \hat{\sigma}_{\lambda N} & \hat{\sigma}_{\lambda p} \\
\hat{\sigma}_{\mathrm{N} \lambda} & \sigma_{\mathrm{N}}^{2} & \hat{\sigma}_{\mathrm{Np}} \\
\hat{\sigma}_{\mathrm{p} \lambda} & \hat{\sigma}_{\mathrm{pN}} & \sigma_{\mathrm{p}}^{2}
\end{array}\right]
$$

Considerando a aproximação Normal Assintótica para os estimadores de máxima verossimilhança $\hat{\lambda}, \hat{\mathrm{N}}$ e $\hat{\mathrm{p}}$, intervalos de confiança para $\lambda$, $\mathrm{N}$ e p com $100(1-\gamma) \%$ de confiança podem ser construídos, dados por:

$$
\begin{aligned}
& \operatorname{ic}(\lambda)=\hat{\lambda}-Z_{\gamma / 2} \hat{\sigma}_{\lambda} ; \hat{\lambda}+Z_{\gamma / 2} \hat{\sigma}_{\lambda,}, \\
& \operatorname{ic}(N)=\hat{N}-Z_{\gamma / 2} \hat{\sigma}_{N} ; \hat{N}+Z_{\gamma / 2} \hat{\sigma}_{N}, \\
& \operatorname{ic}(p)=\hat{p}-Z_{\gamma / 2} \hat{\sigma}_{p} ; \hat{p}+Z_{\gamma / 2} \hat{\sigma}_{p},
\end{aligned}
$$

onde $Z_{\gamma / 2}$ é um percentil da distribuição Normal padronizada.

\subsection{O Modelo de Schick e Wolverton (1978)}

O modelo de Schick e Wolverton (SW) (1978) também faz uso da estratégia tipo I-1, que modela os tempos entre falhas do software, mas desta vez a taxa de falha é assumida ser proporcional ao número de erros que não foram corrigidos (que restam) no sistema e o tempo decorrido da última falha.

Este modelo difere dos modelos já mencionados anteriormente pelo fato de que a taxa de falha não decresce monotonicamente. Imediatamente após a i-ésima falha, a taxa de falha cai para 
zero e então aumenta linearmente com inclinação $(\mathrm{N}$ - i ) a (i + 1)-ésima falha. (veja figura 3 que mostra a taxa de falha para o modelo de Schick e Wolverton).

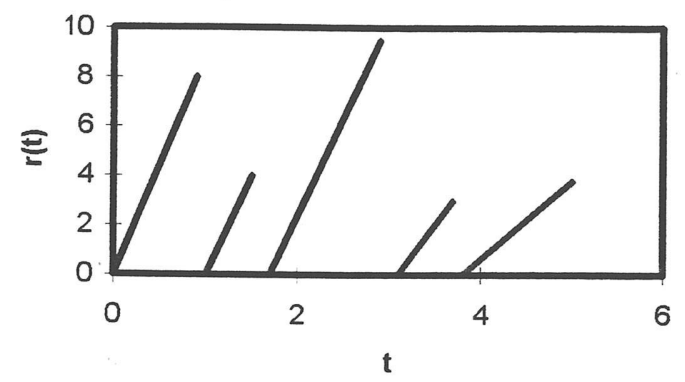

Figura 3: Taxa da falha para o modelo SW

\subsubsection{Formulação do Modelo de Schick e Wolverton}

O modelo de Schick e Wolverton é formulado considerando o número de erros restantes no sistema $(\mathrm{N})$ e o tempo decorrido da última falha $(\mathrm{t})$, ou seja, a taxa de falha para esse modelo é dada por,

$$
\lambda_{\mathrm{i}}=\lambda(\mathrm{N}-\mathrm{i}+1) \mathrm{t}
$$

Sabendo que $T_{1}, \ldots, T_{n}$ (tempos entre falhas do software) são variáveis aleatórias com distribuição Exponencial, assumindo o modelo de Schick e Wolverton, podemos observar que a função densidade para os tempos entre falha $T_{i}$ se torna:

$$
f_{i}(t)=\lambda t(N-i+1) \exp \left\{-\lambda(N-i+1) \frac{t^{2}}{2}\right\} .
$$

Assumindo $T_{1}, \ldots, T_{n}$ variáveis aleatórias independentes, a função de verossimilhança para $\lambda$ e $\mathrm{N}$ é dada por, 


$$
L(\lambda, N)=\prod_{i=1}^{n} f\left(t_{i} \mid \lambda_{i}\right)
$$

onde $\lambda_{\mathrm{i}}=\lambda \mathrm{t}(\mathrm{N}-\mathrm{i}+1), \mathrm{i}=1,2, \ldots, \mathrm{t} \geq 0$.

Desenvolvendo (2.71), obtemos:

$$
L(\lambda, N)=\lambda^{n} \prod_{i=1}^{n} t_{i}(N-i+1) \exp \left\{-\frac{\lambda}{2} \sum_{i=1}^{n}(N-i+1) t_{i}^{2}\right\}
$$

Definindo $A_{1}(N)=\prod_{i=1}^{n}(N-i+1) t_{i}$ e $B_{1}(N)=\sum_{i=1}^{n}(N-i+1) t_{i}^{2}$, temos:

$$
L(\lambda, N)=\lambda^{n} A_{1}(N) \exp \left\{-\frac{\lambda}{2} B_{1}(N)\right\}
$$

\subsubsection{Estimadores de Máxima Verossimilhança para os Parâmetros $\lambda$ e N}

Através do logaritmo da função de máxima verossimilhança, podemos obter os estimadores de máxima verossimilhança de $\lambda$ e $N$.

O logarítmo da função de verossimilhança (2.72) é dado por,

$$
1(\lambda, N)=n \log \lambda+\sum_{i=1}^{n} \ln \left[t_{i}(N-i+1)\right]-\frac{\lambda}{2} \sum_{i=1}^{n}(N-i+1) t_{i}^{2}
$$

Considerando as primeiras derivadas de $1(\lambda, N)$ com respeito a $\lambda$ e $N$, dadas por, 


$$
\begin{aligned}
& \frac{\partial \mathrm{l}(\lambda, N)}{\partial \lambda}=\frac{\mathrm{n}}{\lambda}-\sum_{\mathrm{i}=1}^{\mathrm{n}}(\mathrm{N}-\mathrm{i}+1) \frac{\mathrm{t}_{\mathrm{i}}^{2}}{2}, \\
& \frac{\partial \mathrm{l}(\lambda, N)}{\partial \mathrm{N}}=\sum_{\mathrm{i}=1}^{\mathrm{n}}\left(\frac{1}{N-\mathrm{i}+1}\right)-\frac{\lambda}{2} \sum_{\mathrm{i}=1}^{\mathrm{n}} \mathrm{t}_{\mathrm{i}}^{2},
\end{aligned}
$$

e igualando-as a zero, obtemos os estimadores de máxima verossimilhança de $\lambda$ e $\mathrm{N}$ dados pelas soluções das seguintes equações:

$$
\hat{\lambda}=\frac{2 \mathrm{n}}{\hat{\mathrm{N}} \sum_{\mathrm{i}=1}^{\mathrm{n}} \mathrm{t}_{\mathrm{i}}^{2}-\sum_{\mathrm{i}=1}^{\mathrm{n}}(\mathrm{i}-1) \mathrm{t}_{\mathrm{i}}^{2}},
$$

e N̂́ solução da equação:

$$
\sum_{i=1}^{n} \frac{1}{(N-i+1)}=\frac{n}{N-\frac{1}{S_{n}} \sum_{i=1}^{n}(i-1) t_{i}^{2}}
$$

onde $\mathrm{S}_{\mathrm{n}}=\sum_{\mathrm{i}=1}^{\mathrm{n}} \mathrm{t}_{\mathrm{i}}^{2}$

Devido as equações (2.76) e (2.77) serem não lineares, suas soluções podem ser obtidas com o auxílio de métodos iterativos, como por exemplo o método de Newton-Raphson, (ver po exemplo, Zacks, 1971).

\subsubsection{Inferências Sobre os Parâmetros $\lambda$ e N}

Como já mencionado anteriormente, utilizaremos a aproximação Normal assintótica dos estimadores de máxima verossimilhança para fazermos inferências sobre os parâmetros $\lambda$ e $\mathrm{N}$ no modelo de Schick e Wolverton (ver por exemplo Sprott, 1973, 1980). Portanto consideramos, 


$$
(\hat{\lambda}, \hat{\mathrm{N}}) \stackrel{\mathrm{a}}{\sim} \mathrm{N}\left\{(\lambda, \mathrm{N}) ; \mathrm{I}_{\mathrm{o}}^{-1}(\hat{\lambda}, \hat{\mathrm{N}})\right\}
$$

onde $I_{0}(\lambda, N)$ é a matriz de informação observada, dada pelas segundas derivadas do logarítmo da verossimilhança (2.73) com respeito a $\lambda$ e $N$, a menos do sinal (ver por exemplo, Box e Tiao, 1973).

As segundas derivadas da função (2.73) com respeito a $\lambda$ e $N$ são dadas por,

$$
\begin{gathered}
\frac{\partial^{2} 1(\lambda, N)}{\partial \lambda^{2}}=-\frac{n}{\lambda^{2}}, \\
\frac{\partial^{2} 1(\lambda, N)}{\partial \lambda \partial N}=-\sum_{i=1}^{n} \frac{t_{i}^{2}}{2}, \\
\frac{\partial^{2} l(\lambda, N)}{\partial N^{2}}=-\sum_{i=1}^{n}\left[\frac{1}{(N-i+1)^{2}}\right] .
\end{gathered}
$$

Por se inviável obter a esperança dos tempos entre falhas $\left(E\left(T_{i}\right)\right)$, no modelo de Schick e Wolverton, utilizaremos a matriz de informação observada $I_{0}(\lambda, N)$, dada pelas segundas derivadas do logarítmo da verossimilhança descritas em $2.79,2.80$ e 2.81 a menos do sinal, dada por,

$$
I_{0}(\lambda, N)=\left[\begin{array}{cc}
\frac{n}{\lambda^{2}} & \sum_{i=1}^{n} \frac{t_{i}{ }^{2}}{2} \\
\sum_{i=1}^{n} \frac{t_{i}^{2}}{2} & \sum_{i=1}^{n} \frac{1}{(N-i+1)^{2}}
\end{array}\right] .
$$

$\mathrm{I}_{\mathrm{o}}{ }^{-1}(\hat{\lambda}, \hat{\mathrm{N}})$, o inverso da matriz de Informação observada, é a matriz de variâncias e covariâncias assintótica de $\hat{\lambda}$ e $\hat{N}$, dada por, 


$$
\mathrm{I}_{\mathrm{o}}{ }^{-1}(\hat{\lambda}, \hat{\mathrm{N}})=\left[\begin{array}{cc}
\hat{\sigma}_{\lambda}^{2} & \hat{\sigma}_{\lambda N} \\
\hat{\sigma}_{\lambda N} & \hat{\sigma}_{\mathrm{N}}^{2}
\end{array}\right]
$$

(ver por exemplo, Bickel \& Doksum, 1977).

Considerando a distribuição Normal Assintótica (2.78) para os estimadores de máxima verossimilhança $\hat{\lambda}$ e $\hat{\mathrm{N}}$, é possível construir intervalos de confiança para os parâmetros $\lambda$ e $\mathrm{N}$.

Os intervalos de confiança para $\lambda$ e $N$, considerando um nível de confiança $100(1-\gamma) \%$, são dados por,

$$
\begin{aligned}
& \operatorname{ic}(\lambda)=\hat{\lambda}-Z_{\gamma / 2} \hat{\sigma}_{\lambda} ; \hat{\lambda}+Z_{\gamma / 2} \hat{\sigma}_{\lambda,} \\
& \operatorname{ic}(N)=\hat{N}-Z_{\gamma / 2} \hat{\sigma}_{N} ; \hat{N}+Z_{\gamma / 2} \hat{\sigma}_{N},
\end{aligned}
$$

onde $Z_{\gamma / 2}$ é um percentil da distribuição Normal padronizada. 
Capítulo 3

\section{UMA ANÁLISE BAYESIANA PARA OS PRINCIPAIS MODELOS DE ESTRATÉGIA TIPO I}

Em análise de confiabilidade de software, quando assumimos um modelo para os tempos entre falhas do software, temos interesse em determinar o valor do parâmetro que o define. Embora este parâmetro seja considerado fixo, não temos certeza do seu verdadeiro valor e assim procuramos uma forma de, através dos dados, expressar essa nossa incerteza.

Uma alternativa é considerar uma distribuição para o parâmetro de interesse, que sob este ponto de vista é considerado uma variável aleatória. Com esta formulação, usamos o teorema de Bayes, (ver por exemplo Box e Tiao, 1973), que fornece um método para computar a densidade de probabilidade do parâmetro, expressando nossa incerteza condicionada aos dados observados.

\subsection{Fórmula de Bayes}

A inferência Bayesiana, assim como a inferência frequentista, trabalham com a presença de observações $\mathrm{x}$, inicialmente incertas e descritas através de uma distribuição de probabilidade com densidade de probabilidade $\mathrm{f}(\mathrm{x} \mid \theta)$. A quantidade $\theta$, sob o ponto de vista Bayesiano, é considerado uma variável aleatória, representando características de interesse que se deseja conhecer para poder ter uma descrição completa do processo.

Neste sentido considerando, $\mathrm{x}$ e $\theta$ variáveis aleatórias com densidades conjuntas e condicionais $\mathrm{p}(\mathrm{x}, \theta), \mathrm{f}(\mathrm{x} \mid \theta)$ e $\mathrm{p}(\theta \mid \mathrm{x})$, respectivamente, e $\mathrm{p}(\theta)$ a densidade marginal de $\theta$, a densidade condicional de $\theta$ dado $\mathrm{x}$, é dada por,

$$
p(\theta \mid x)=\frac{f(x \mid \theta) p(\theta)}{f(x)}
$$


onde $f(x)=\int f(x \mid \theta) p(\theta) d \theta$

Denotando a função de verossimilhança $\mathrm{f}(\mathrm{x} \mid \theta)$ por $\mathrm{L}(\theta)$, a densidade a priori por $\pi(\theta)$ e a densidade a posteriori por $\pi(\theta \mid \mathrm{D})$, o teorema de Bayes pode ser escrito em uma forma mais simplificada, como,

$$
\pi(\theta \mid \mathrm{D}) \propto \mathrm{L}(\theta) \pi(\theta)
$$

onde D denota o conjunto de observações x.

\subsection{Densidades a Priori e a Posteriori}

No método Bayesiano é possível incorporar um conhecimento prévio a respeito da quantidade $\theta$ à análise, através de uma densidade $\pi(\theta)$, sendo essa densidade identificada como densidade a priori.

Uma vez definido o modelo para os dados e a distribuição a priori, devemos combinar a informação prévia sobre o parâmetro $\theta$ com a informação contida nos dados, obtendo assim a distribuição a posteriori para $\theta$

O processo de inferência baseia-se na distribuição a posteriori de $\theta$. Portanto, uma vez obtida essa distribuição, podemos sumarizar a informação nela contida através de alguns elementos como as medidas de posição (média, moda e mediana) e medidas de dispersão (variância, desvio - padrão, etc.).

Uma das limitações dos métodos Bayesianos nas aplicações, em geral eram relacionadas à resolução de integrais Bayesianas para obtenção das distribuições marginais, que muitas vezes não apresentam soluções analíticas explícitas e exigem o uso de métodos de aproximação como por exemplo, o método de Laplace para aproximações de integrais, (ver por exemplo, Tierney e Kadane, 1986).

Neste sentido, uma alternativa recente, é utilizar técnicas de simulação, como o método (Gibbs Sampling e Metrópolis Hastings (ver por exemplo, Gelfand e Smith, 1990), de grande 
aplicabilidade para uma análise Bayesiana completa em modelos mais complexos com estrutura hierárquica.

A técnica Gibbs Sampling tem sido muito difundida na literatura (ver por exemplo, Casella e George, 1992; Geman e Geman, 1984; Gelfand e Smith, 1990).

O algorítmo Gibbs Sampling é um método de simulação via cadeias de Markov que fornece uma forma alternativa para se obter uma amostra da distribuição de interesse (se está supondo que a geração direta dessa distribuição é extremamente complicada e/ou custosa) baseada em sucessivas gerações de distribuições condicionais ( ver apêndice 1).

No caso em que se sabe gerar valores de todas as distribuições condicionais, ou seja, quando conseguimos identificar distribuições conhecidas, utilizamos o algorítmo Gibbs Sampling. Mas geralmente, em modelos mais complexos é possível estabelecer conjugação condicional para algumas mas não para todas componentes do modelo.

Nesse caso, sugere-se que a geração das amostras sejam feitas através do algorítmo Metrópolis Hastings (ver apêndice 2).

A convergência da amostra à distribuição de equilíbrio pode ser monitorada pelo método proposto por Gelman e Rubin (1992), (ver apêndice 3).

Neste capítulo, apresentamos uma análise Bayesiana utilizando os algorítmos Gibbs Sampling e Metrópolis Hastings para os modelos de Jelinski e Moranda (1972), Moranda (1975), Goel e Okumoto (1978), Schick e Wolverton (1978) e para o modelo Bayesino de Littlewood e Verral (1973) que será definido na seção 3.8.

Diferentes densidades a priori para os parâmetros do modelo de Jelinski e Moranda são consideradas, englobando densidades a priori hierárquicas, onde os hiperparâmetros (os parâmetros das distribuições dos parâmetros) do modelo são desconhecidos com uma distribuição conhecida.

\subsection{Uma Análise Bayesiana para o Modelo de Jelinski e Moranda}

Nesta seção, assumindo dados sobre tempos entre falhas do software descritos por uma distribuição Exponencial e considerando diferentes densidades a priori, englobando densidades a priori hierárquicas, para $\lambda$ e $\mathrm{N}$, desenvolvemos uma análise Bayesiana usando o algorítmo Gibbs Sampling com Metrópolis Hastings. 


\subsubsection{Densidades a Priori assumindo $\lambda$ com Distribuição Gama e N com Distribuição de Poisson}

Para representarmos o grau de conhecimento sobre os parâmetros $\lambda$ e $\mathrm{N}$ no modelo de $\mathrm{JM}$, dado o espaço paramétrico em que se encontram, consideramos uma densidade a priori para $\lambda$ e $N$, dadas respectivamente por:

$$
\begin{gathered}
\lambda \sim \Gamma\left[\mathrm{a}_{1}, \mathrm{~b}_{1}\right], \\
\mathrm{N} \sim \mathrm{P}\left(\theta_{1}\right),
\end{gathered}
$$

onde $\Gamma\left[\mathrm{a}_{1}, \mathrm{~b}_{1}\right]$ denota a distribuição Gama com média $\mathrm{a}_{1} / \mathrm{b}_{1}, \mathrm{P}\left(\theta_{1}\right)$ denota a distribuição Poisson com média $\theta_{1}$ e $a_{1}, b_{1}$ e $\theta_{1}$ são constantes conhecidas baseadas na informação a priori do especialista.

Assumindo independência a priori, a densidade a priori conjunta para $\lambda$ e $\mathrm{N}$ é dada por,

$$
\pi(\lambda, N) \propto \frac{e^{-\theta_{1}} \theta_{1}^{N}}{N !} \lambda^{a_{1}-1} e^{-b_{1} \lambda}
$$

onde $\lambda>0$ e $N=n, n+1, \ldots$

Assumindo o modelo de falha truncada, isto é, assumindo que o teste do software termina quando se observa a n-ésima falha e considerando $T_{1}, \ldots, T_{n}$ uma amostra aleatória dos tempos entre falhas sob o modelo de JM (2.1), vamos supor que o teste do software é feito até a $\mathrm{x}_{\mathrm{n}}$-ésima estatística de ordem, ou seja, enfatizamos o tempo de falha $x_{i}$, onde $t_{i}=x_{i}-x_{i-1}$.

Dessa forma,

$$
B(N)=\sum_{i=1}^{n}(N-i+1) t_{i}=\sum_{i=1}^{n} x_{i}+(N-n) x_{n} .
$$


De (3.3) a função de verossimilhança para $\lambda$ e $\mathrm{N}$ é dada por,

$$
L(\lambda, N)=\lambda^{n} A(N) \exp \left\{-\lambda\left[\sum_{i=1}^{n} x_{i}+(N-n) x_{n}\right]\right\}
$$

onde $A(N)=\prod_{i=1}^{n}(N-i+1)$.

Considerando a densidade a priori (3.2) e a função de verossimilhança (3.4), a densidade a posteriori conjunta para $\lambda$ e $\mathrm{N}$ é dada por, (ver por exemplo, Box e Tiao, 1973)

$$
\pi(\lambda, N \mid D) \propto \pi(\lambda, N) L(\lambda, N)
$$

isto é,

$$
\pi(\lambda, N \mid D) \propto \lambda^{n+a_{1}-1} \frac{A(N) \theta_{1}^{N}}{N !} \exp \left\{-\left[b_{1}+(N-n) x_{n}+\sum_{i=1}^{n} x_{i}\right] \lambda\right\},
$$

onde $\lambda>0, N=n, n+1, \ldots$ e $D$ denota o conjunto de dados sobre tempos de falhas $\left\{\mathrm{x}_{1}, \mathrm{x}_{2}, \ldots\right.$, $\mathrm{x}_{\mathrm{n}}$.

Em inferência Bayesiana, inferências são tipicamente baseadas nas densidades a posteriori marginais dos parâmetros envolvidos.

Para obtermos uma amostra das densidades a posteriori marginais de $\lambda$ e $\mathrm{N}$, exploramos o uso do método Gibbs Sampling (ver apêndice 1) que baseia-se em sucessivas gerações das distribuições condicionais a posteriori $(\lambda \mid N, D)$ e $(N \mid \lambda, D)$.

Dessa forma, considerando a densidade a posteriori conjunta (3.5), a densidade a posteriori condicional de $\lambda$ requerida para o algorítmo Gibbs Sampling pode ser obtida por, 


$$
\pi(\lambda \mid N, D)=\frac{\pi(\lambda, N \mid D)}{\pi(N \mid D)}
$$

onde $\pi(\mathrm{N} \mid \mathrm{D})$ é a densidade a posteriori marginal de $\mathrm{N}$, que pode ser obtida integrando-se a densidade a posteriori conjunta (3.5) com respeito a $\lambda$. Portanto,

$$
\lambda \mid \mathrm{N}, \mathrm{D} \sim \Gamma\left[\mathrm{n}+\mathrm{a}_{1}, \mathrm{~b}_{1}+\mathrm{B}(\mathrm{N})\right]
$$

onde $\Gamma\left[n+a_{1}, b_{1}+B(N)\right]$ denota a distribuição Gama com média $\frac{\left(n+a_{1}\right)}{\left(b_{1}+B(N)\right)}$ e $B(N)$ é dado na expressão (3.3)

A distribuição condicional para $\mathrm{N}$ dado $\lambda$ e os dados (D), requerida para o algorítmo Gibbs Sampling, pode ser obtida por,

$$
\pi(N \mid \lambda, D)=\frac{\pi(\lambda, N \mid D)}{\pi(\lambda \mid D)}
$$

onde $\pi(\lambda \mid \mathrm{D})$ é a densidade a posteriori marginal de $\lambda$, obtida somando-se a densidade $a$ posteriori conjunta (3.5) com respeito a N. Portanto,

$$
\pi(N \mid \lambda, D) \propto \frac{A(N)}{N !} \theta_{1}^{N} e^{-(N-n) x_{n} \lambda}
$$

Podemos observar que $\frac{A(N)}{N !}=\frac{1}{(N-n) !}$. Dessa forma, se definirmos a transformação $\mathrm{N}^{\prime}=\mathrm{N}-\mathrm{n}$, obtemos a distribuição Poisson com média $\theta_{1} \mathrm{e}^{-\mathrm{x}_{\mathrm{n}} \lambda}$ para $\mathrm{N}^{\prime}$ dado $\lambda$ e os dados (D).

Assim, as densidades condicionais de $\left(\lambda \mid N^{\prime}, D\right)$ e $\left(N^{\prime} \mid \lambda, D\right)$ requeridas para o algorítmo Gibbs Sampling são dadas por, 


$$
\begin{gathered}
\lambda \mid \mathrm{N}^{\prime}, \mathrm{D} \sim \Gamma\left[\mathrm{n}+\mathrm{a}_{1}, \mathrm{~b}_{1}+\mathrm{N}^{\prime} \mathrm{x}_{\mathrm{n}}+\sum_{\mathrm{i}=1}^{\mathrm{n}} \mathrm{x}_{\mathrm{i}}\right], \\
\mathrm{N}^{\prime} \mid \lambda, \mathrm{D} \sim \mathrm{P}\left(\theta_{1} \mathrm{e}^{-\mathrm{x}_{\mathrm{n}} \lambda}\right) .
\end{gathered}
$$

\subsubsection{Densidades a Priori assumindo $\lambda$ com Distribuição Gama e N com Distribuição Binomial Negativa}

Consideramos nesta sub-seção, dado o espaço paramétrico dos parâmetros, outras densidades a priori para $\lambda$ e N sob o modelo de Jelinski e Moranda (2.1).

Assumindo independência a priori, consideramos as seguintes densidades a priori para $\lambda$ e N:

$$
\begin{gathered}
\lambda \sim \Gamma[\gamma, \delta], \\
\mathrm{N} \sim \mathrm{BN}[\mathrm{r}, \mathrm{p}],
\end{gathered}
$$

onde,

$$
\pi(N) \propto\left(\begin{array}{c}
N+r-1 \\
r-1
\end{array}\right) p^{r}(i-p)^{n}
$$

onde $\Gamma[\gamma, \delta]$ denota a distribuição Gama com média $\gamma / \lambda, \operatorname{BN}[\mathrm{r}, \mathrm{p}]$ denota a distribuição Binomial Negativa com média r/p cuja expressão está descrita na equação (3.9) e $\gamma, \delta, r$ e p são constantes conhecidas baseadas na informação a priori do especialista.

Dessa forma, a densidade a priori conjunta para $\lambda$ e $\mathrm{N}$ é dada por,

$$
\pi(\lambda, N) \propto\left(\begin{array}{c}
N+r-1 \\
r-1
\end{array}\right) \lambda^{\gamma-1} e^{-\delta \lambda} p^{r}(1-p)^{N}
$$


onde $\lambda>0$ e $N=n, n+1, \ldots$

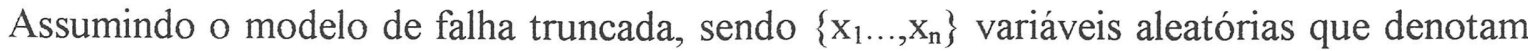
os tempos de falha sob o modelo de JM (2.1), a densidade a priori (3.10) e a função de máxima verossimilhança (3.4), a densidade a posteriori conjunta para $\lambda$ e $N$ é dada por,

$$
\pi(\lambda, N \mid D) \propto \lambda^{n+\gamma-1} A(N)\left(\begin{array}{c}
N+r-1 \\
r-1
\end{array}\right)(1-p)^{N} \exp \{-\lambda[\delta+B(N)]\}
$$

onde $A(N)=\prod_{i=1}^{n}(N-i+1), B(N)=(N-n) x_{n}+\sum_{i=1}^{n} x_{i}$ e D denota o conjunto de dados dos tempos de falha $\left\{\mathrm{x}_{1}, \ldots, \mathrm{x}_{\mathrm{n}}\right\}$.

Assumindo a densidade a posteriori conjunta (3.11), a densidade a posteriori condicional requerida para o algorítmo Gibbs Sampling para $\lambda$ dado $N$ e os dados (D) é dada por,

$$
\lambda \mid \mathrm{N}, \mathrm{D} \sim \Gamma\left[\mathrm{n}+\gamma, \delta+(\mathrm{N}-\mathrm{n}) \mathrm{x}_{\mathrm{n}}+\sum_{\mathrm{i}=1}^{\mathrm{n}} \mathrm{x}_{\mathrm{i}}\right],
$$

onde $\Gamma[n+\gamma, \delta+B(N)]$ denota a distribuição Gama com média $(n+\gamma) /[\delta+B(N)]$.

A distribuição a posteriori condicional para $\mathrm{N}$ dado $\lambda$ e os dados (D) requerida para o algorítmo Gibbs Sampling, é dada por,

$$
\pi(N \mid \lambda, D) \propto A(N)\left(\begin{array}{c}
N+r-1 \\
r-1
\end{array}\right)(1-p)^{N} e^{-(N-n) \lambda x_{n}}
$$

Definindo a transformação $\mathrm{N}^{\prime}=\mathrm{N}-\mathrm{n}$, podemos notar que,

$$
A\left(N^{\prime}\right)\left(\begin{array}{c}
N^{\prime}+n+r-1 \\
r-1
\end{array}\right) \propto \frac{\left(N^{\prime}+n+r-1\right) !}{N^{\prime} !},
$$


onde $A\left(N^{\prime}\right)=\prod_{i=1}^{n}\left(N^{\prime}+n-i+1\right)$ e $\left(\begin{array}{c}N^{\prime}+n+r-1 \\ r-1\end{array}\right)=\frac{\left(N^{\prime}+n+r-1\right) !}{\left(N^{\prime}+n\right) !}$

Dessa forma, obtemos:

$$
\pi\left(N^{\prime} \mid \lambda, D\right) \propto\left(\begin{array}{c}
N^{\prime}+n+r-1 \\
n+r-1
\end{array}\right)\left[1-(1-p) e^{-\lambda x_{n}}\right]^{n+r}\left[(1-p) e^{-\lambda x_{n}}\right]^{N^{\prime}}
$$

que é a distribuição Binomial Negativa com média $(n+r) /\left[1-(1-p) e^{-\lambda x_{n}}\right]$.

Assim considerando a transformação $N^{\prime}=N-n$, as densidades condicionais de $\lambda \mid N^{\prime}, D$ e $\mathrm{N}^{\prime} \mid \lambda, \mathrm{D}$, requeridas para o algorítmo Gibbs Sampling podem ser dadas por,

$$
\begin{aligned}
& \lambda \mid \mathrm{N}^{\prime}, \mathrm{D} \sim \Gamma\left[\mathrm{n}+\gamma, \delta+\mathrm{N}^{\prime} \mathrm{x}_{\mathrm{n}}+\sum_{\mathrm{i}=1}^{\mathrm{n}} \mathrm{x}_{\mathrm{i}}\right], \\
& \mathrm{N}^{\prime} \mid \lambda, \mathrm{D} \sim \mathrm{BN}\left[\mathrm{n}+\mathrm{r}, 1-(1-\mathrm{p}) \mathrm{e}^{-\lambda \mathrm{x}_{\mathrm{n}}}\right] .
\end{aligned}
$$

\subsubsection{Densidades a Priori assumindo $\lambda$ com Distribuição Gama e N com Distribuição Logarítmica}

Considerando o modelo de Jelinski e Moranda (2.1), apresentamos nessa seção uma análise Bayesiana enfatizando um terceiro grupo de densidades a priori para $\lambda$ e $N$.

Assumindo independência a priori, consideramos uma densidade a priori para $\lambda$ dada por,

$$
\lambda \sim \Gamma\left[\gamma_{1}, \delta_{1}\right]
$$


onde $\Gamma\left[\gamma_{1}, \delta_{1}\right]$ denota a distribuição Gama com média $\gamma_{1} / \delta_{1}$ e $\gamma_{1}$ e $\delta_{1}$ são constantes conhecidas.

Para N, assumimos uma densidade a priori com distribuição logarítmica (ver por exemplo Yang, 1994), onde,

$$
f(N \mid \theta)=\frac{\theta^{N}}{N(-\ln (1-\theta))}
$$

cuja média é dada por,

$$
E(N)=\frac{1}{(1-\theta)[-\ln (1-\theta)]}
$$

onde $0<\theta<1$.

Dessa forma de (3.16) e (3.17), a densidade a priori conjunta para $\lambda$ e $\mathrm{N}$ é dada por,

$$
\pi(\lambda, N) \propto \frac{\lambda^{\gamma_{1}-1} \theta^{N}}{N[-\ln (1-\theta)]} \exp \left\{-\delta_{1} \lambda\right\}
$$

onde $\lambda>0,0<\theta<1$ e $\mathrm{N}=\mathrm{n}, \mathrm{n}+1, \ldots$

Assumindo a densidade a priori (3.19) e a função de máxima verossimilhança (3.4) sob o modelo de falha truncada de JM (2.1), a densidade a posteriori conjunta para $\lambda$ e N é dada por,

$$
\pi(\lambda, N \mid D) \propto \lambda^{n+\gamma_{1}-1} A(N) \frac{\theta^{N}}{N[-\ln (1-\theta)]} \exp \left\{-\left[\delta_{1}+B(N)\right] \lambda\right\},
$$


onde $A(N)=\prod_{i=1}^{n}(N-i+1), B(N)=(N-n) x_{n}+\sum_{i=1}^{n} x_{i}$ e D denota os tempos de falha $\left\{x_{1}, \ldots\right.$, $\left.\mathrm{x}_{\mathrm{n}}\right\}$.

Assumindo a densidade a posteriori conjunta (3.20), a densidade a posteriori condicional de $\lambda$ dado $\mathrm{N}$ e os dados (D) requerida como núcleo de transição no algorítmo Gibbs Sampling, é dada por,

$$
\lambda \mid \mathrm{N}, \mathrm{D} \sim \Gamma\left[\mathrm{n}+\gamma_{1}, \delta_{1}+(\mathrm{N}-\mathrm{n}) \mathrm{x}_{\mathrm{n}}+\sum_{\mathrm{i}=1}^{\mathrm{n}} \mathrm{x}_{\mathrm{i}}\right],
$$

onde $\Gamma\left[\mathrm{n}+\gamma_{1}, \delta_{1}+(\mathrm{N}-\mathrm{n}) \mathrm{x}_{\mathrm{n}}+\sum_{\mathrm{i}=1}^{\mathrm{n}} \mathrm{x}_{\mathrm{i}}\right]$ denota a distribuição Gama com média $\left(\mathrm{n}+\gamma_{1}\right) /\left[\delta_{1}+(\mathrm{N}-\mathrm{n}) \mathrm{x}_{\mathrm{n}}+\sum_{\mathrm{i}=1}^{\mathrm{n}} \mathrm{x}_{\mathrm{i}}\right]$

A distribuição a posteriori condicional para $\mathrm{N}$ dado $\lambda$ e os dados (D), requerida para o algorítmo Gibbs Sampling, é dada por,

$$
\pi(N \mid \lambda, D) \propto A(N) \frac{\theta^{N}}{N[-\ln (1-\theta)]} e^{-(N-n) x_{n} \lambda} .
$$

Definindo a transformação $\mathrm{N}^{\prime}=\mathrm{N}-\mathrm{n}$, temos,

$$
\pi\left(N^{\prime} \mid \lambda, D\right) \propto A\left(N^{\prime}\right) \frac{\theta^{N^{\prime}+n}}{\left(N^{\prime}+n\right)[-\ln (1-\theta)]} e^{-N^{\prime} \lambda x_{n}},
$$

onde $A\left(N^{\prime}\right) \frac{1}{\left(N^{\prime}+n\right)}=\frac{\left(N^{\prime}+n-1\right) !}{N^{\prime} !}=\left(\begin{array}{c}N^{\prime}+n-1 \\ n-1\end{array}\right)$

Dessa forma, de (3.22) obtemos: 


$$
\pi\left(N^{\prime} \mid \lambda, D\right) \propto\left(\begin{array}{c}
N^{\prime}+n-1 \\
n-1
\end{array}\right)\left(1-\theta e^{-\lambda x_{n}}\right)^{n}\left(\theta e^{-\lambda x_{n}}\right)^{N^{\prime}}
$$

que é a distribuição Binomial Negativa com parâmetros (n) e $\left(1-\theta \mathrm{e}^{-\lambda \mathrm{x}_{\mathrm{n}}}\right)$, onde $\mathrm{N}^{\prime}=0,1, \ldots$ e $0<\theta<1$.

Assim, temos as densidades condicionais de $\lambda \mid N^{\prime}, D$ e $N^{\prime} \mid \lambda, D$, requeridas para o algorítmo Gibbs Sampling, dadas por,

$$
\begin{gathered}
\lambda \mid \mathrm{N}^{\prime}, \mathrm{D} \sim \Gamma\left[\mathrm{n}+\gamma_{1}, \delta_{1}+\mathrm{N}^{\prime} \mathrm{x}_{\mathrm{n}}+\sum_{\mathrm{i}=1}^{\mathrm{n}} \mathrm{x}_{\mathrm{i}}\right], \\
\mathrm{N}^{\prime} \mid \lambda, \mathrm{D} \sim \mathrm{BN}\left[\mathrm{n}, 1-\theta \mathrm{e}^{-\lambda \mathrm{x}_{\mathrm{n}}}\right] .
\end{gathered}
$$

\subsubsection{Uma Análise Bayesiana Hierárquica para o Modelo de Jelinski e Moranda.}

$\mathrm{Na}$ análise Bayesiana hierárquica, os hiperparâmetros, ou seja, os parâmetros das distribuições dos parâmetros, são desconhecidos com uma distribuição conhecida.

Muitas vezes existem relações probabilísticas a respeito dos parâmetros e hiperparâmetros que podem e devem ser incorporadas ao modelo através de densidades a priori hierárquicas.

Nesta sub-seção apresentamos dois casos de densidades a priori hierárquicas para os parâmetros do modelo de Jelinski e Moranda. 
$1^{\circ}$ caso: $\mathbf{N} \mid \theta \sim \mathbf{P}(\theta), \theta \sim \Gamma[\alpha, \beta]$ e $\lambda \sim \Gamma\left[\gamma_{2}, \delta_{2}\right]$

Assumindo independência a priori, consideramos uma densidade a priori hierárquica para $\mathrm{N}$, dada por,

$$
\begin{aligned}
& \mathrm{N} \mid \theta \sim \mathrm{P}(\theta), \\
& \theta \sim \Gamma[\alpha, \beta],
\end{aligned}
$$

onde $\mathrm{P}(\theta)$ denota a distribuição Poisson, cuja média é dada por $\theta, \Gamma[\alpha, \beta]$ denota a distribuição Gama com média $\alpha / \beta$ e $\alpha$ e $\beta$ são constantes conhecidas.

A densidade a priori para $\lambda$ é dada por,

$$
\lambda \sim \Gamma\left(\gamma_{2}, \delta_{2}\right)
$$

onde $\Gamma\left(\gamma_{2}, \delta_{2}\right)$ denota a distribuição Gama com média $\gamma_{2} / \delta_{2}$ sendo $\gamma_{2}$ e $\delta_{2}$ constantes conhecidas.

Portanto, de (3.25) e (3.26), a densidade a priori conjunta para $\lambda, \mathrm{N}$ e $\theta$ é dada por,

$$
\pi(\lambda, N, \theta) \propto \frac{\theta^{\mathrm{N}+\alpha-1} \lambda^{\gamma_{2}-1}}{\mathrm{~N} !} \exp \left\{-(1+\beta) \theta-\delta_{2} \lambda\right\}
$$

onde $\lambda>0$, e $N=n, n+1, \ldots$ e $\theta>0$.

Assumindo o modelo de Jelinski e Moranda (2.1), a densidade a priori (3.27) e a função de máxima verossimilhança (3.4), a densidade a posteriori conjunta para $\lambda, \mathrm{N}$ e $\theta$ é dada por, 


$$
\pi(\lambda, N, \theta \mid D) \propto \lambda^{n+\gamma_{2}-1} \frac{\theta^{N+\alpha-1}}{N !} A(N) \exp \left\{-\left[\delta_{2}+B(N)\right] \lambda\right\} \exp \{-(\beta+1) \theta\},
$$

onde $A(N)=\prod_{i=1}^{n}(N-i+1), B(N)$ é dado em (3.3) e D denota os tempos de falha $\left\{x_{1}, \ldots, x_{n}\right\}$ sob o modelo de falha truncada.

As densidades marginais condicionais para $\lambda, \mathrm{N}$ e $\theta$ podem ser dadas por,

$$
\begin{gathered}
\lambda \mid \mathrm{N}, \theta, \mathrm{D} \sim \Gamma\left[\gamma_{2}+\mathrm{n}, \delta_{2}+(\mathrm{N}-\mathrm{n}) \mathrm{x}_{\mathrm{n}}+\sum_{\mathrm{i}=1}^{\mathrm{n}} \mathrm{x}_{\mathrm{i}}\right], \\
\pi(\mathrm{N} \mid \lambda, \theta, \mathrm{D}) \propto \frac{\theta^{\mathrm{N}}}{\mathrm{N} !} \mathrm{A}(\mathrm{N}) \exp \left\{-\left[\gamma_{2}+\mathrm{B}(\mathrm{N})\right] \lambda\right\}, \\
\theta \mid \lambda, \mathrm{N}, \mathrm{D} \sim \Gamma(\mathrm{N}+\alpha, \beta+1) .
\end{gathered}
$$

Utilizando a transformação $\mathrm{N}^{\prime}=\mathrm{N}$-n (como em (3.4.1)), as distribuições condicionais requeridas para o algoritmo de Gibbs Sampling podem ser dadas por,

$$
\begin{gathered}
\mathrm{N}^{\prime} \mid \theta, \lambda, \mathrm{D} \sim \mathrm{P}\left(\theta \mathrm{e}^{-\lambda \mathrm{x}_{\mathrm{n}}}\right), \\
\lambda \mid \mathrm{N}, \theta, \mathrm{D} \sim \Gamma\left[\gamma_{2}+\mathrm{n}, \delta_{2}+\mathrm{N}^{\prime} \mathrm{x}_{\mathrm{n}}+\sum_{\mathrm{i}=1}^{\mathrm{n}} \mathrm{x}_{\mathrm{i}}\right], \\
\theta \mid \mathrm{N}^{\prime}, \lambda, \mathrm{D} \sim \Gamma\left(\alpha+\mathrm{N}^{\prime}+\mathrm{n}, \beta+1\right) .
\end{gathered}
$$




\section{$2^{\circ}$ caso: $\mathbf{N} \mid \theta \sim \mathbf{P}(\theta), \theta \sim \Gamma[\alpha, \beta], \lambda \sim \Gamma\left[\gamma_{2}, \delta_{2}\right]$ e $\delta_{2} \sim \Gamma(\xi, \eta)$}

Assumindo independência a priori, consideramos uma densidade a priori hierárquica para $\lambda$, dada por,

$$
\begin{gathered}
\lambda \sim \Gamma\left[\gamma_{2}, \delta_{2}\right], \\
\delta_{2} \sim \Gamma[\xi, \eta],
\end{gathered}
$$

onde $\Gamma\left[\gamma_{2}, \delta_{2}\right]$ e $\Gamma[\xi, \eta]$ denotam distribuições Gama e $\gamma_{2}$, $\xi$ e $\eta$ são constantes conhecidas baseadas na opinião a priori do especialista.

Considerando a densidade a priori hierárquica (3.25) para $\mathrm{N}$ e a densidade a priori hierárquica para $\lambda(3.31)$, a densidade a priori conjunta para $\mathrm{N}, \lambda, \theta$ e $\delta_{2}$ é dada por,

$$
\pi\left(\mathrm{N}, \lambda, \theta, \delta_{2}\right)=\frac{\theta^{\mathrm{N}+\alpha-1}}{\mathrm{~N} !} \lambda^{\gamma_{2}-1} \delta^{\xi-1} \exp \left\{-(\beta+1) \theta-(\eta+\lambda) \delta_{2}\right\}
$$

onde $\lambda>0, \theta>0, \delta_{2}>0$ e $N=n, n+1, \ldots$.

Assim, sob o modelo de Jelinski e Moranda (2.1), a densidade a posteriori conjunta para $\lambda, \mathrm{N}, \theta$ e $\delta_{2}$ é dada por,

$$
\begin{aligned}
\pi\left(\lambda, N, \theta, \delta_{2} \mid D\right) \propto \lambda^{n+\gamma_{2}-1} \frac{\theta^{N+\alpha-1}}{N !} \mathrm{A}(\mathrm{N}) \delta_{2}{ }^{\xi-1} \times \mathrm{e}^{-\delta_{2}(\lambda+\eta)} \times \\
\times \mathrm{e}^{-(\beta+1) \theta} \exp \left\{-\lambda\left(\sum \mathrm{x}_{\mathrm{i}}+(\mathrm{N}-\mathrm{n}) \mathrm{x}_{\mathrm{n}}\right)\right\}
\end{aligned}
$$

onde $\mathrm{A}(\mathrm{N})=\prod_{\mathrm{i}=1}^{\mathrm{n}}(\mathrm{N}-\mathrm{i}+1)$, e $\mathrm{D}$ denota o conjunto dos tempos de falha $\left\{\mathrm{x}_{1}, \ldots, \mathrm{x}_{\mathrm{n}}\right\}$. 
Como no primeiro caso, definindo a transformação $\mathrm{N}^{\prime}=\mathrm{N}-\mathrm{n}$, as densidades condicionais para $\lambda, N, \theta$ e $\delta_{2}$ requeridas para o algorítmo Gibbs Sampling são dadas por,

$$
\begin{gathered}
\lambda \mid \mathrm{N}^{\prime}, \theta, \delta_{2}, \mathrm{D} \sim \Gamma\left(\gamma_{2}+\mathrm{n}, \delta_{2}+\mathrm{N}^{\prime} \mathrm{x}_{\mathrm{n}}+\sum_{\mathrm{i}=1}^{\mathrm{n}} \mathrm{x}_{\mathrm{i}}\right), \\
\mathrm{N}^{\prime} \mid \theta, \lambda, \delta_{2}, \mathrm{D} \sim \mathrm{P}\left(\theta \mathrm{e}^{-\lambda \mathrm{x}_{\mathrm{n}}}\right), \\
\theta \mid \mathrm{N}^{\prime}, \lambda, \delta_{2}, \mathrm{D} \sim \Gamma\left(\alpha+\mathrm{N}^{\prime}+\mathrm{n}, \beta+1\right), \\
\delta_{2} \mid \lambda, \mathrm{N}^{\prime}, \theta, \mathrm{D} \sim \Gamma\left(\xi+\gamma_{2}, \eta+\lambda\right) .
\end{gathered}
$$

\subsection{Uma Análise Bayesiana para o Modelo de Moranda}

$\mathrm{Na}$ seção 3.4 descrevemos uma análise Bayesiana com o uso do algorítmo Gibbs Sampling com Metrópolis Hastings para o modelo de Moranda (2.22), assumindo os tempos entre falhas do software com uma distribuição Exponencial e considerando densidades a priori informativas para os parâmetros do modelo.

\subsubsection{Densidades a Priori para $\theta$ e K}

Dado o espaço paramétrico dos parâmetros do modelo de Moranda ( $\theta$ e K), sugerimos as seguintes densidades a priori para $\theta$ e K:

$$
\begin{gathered}
\theta \sim \Gamma\left[\mathrm{a}_{2}, \mathrm{~b}_{2}\right], \\
\mathrm{K} \sim \mathrm{B}\left[\mathrm{a}_{3}, \mathrm{~b}_{3}\right],
\end{gathered}
$$


onde $\Gamma\left[\mathrm{a}_{2}, \mathrm{~b}_{2}\right]$ denota uma distribuição Gama com média $\mathrm{a}_{2} / \mathrm{b}_{2}, \mathrm{~B}\left[\mathrm{a}_{3}, \mathrm{~b}_{3}\right]$ denota uma distribuição Beta, $\pi(\mathrm{K}) \propto \mathrm{K}^{\mathrm{a}_{3}-1}(1-\mathrm{K})^{\mathrm{b}_{3}-1}, 0 \leq \mathrm{K} \leq 1$ e $\mathrm{a}_{2}, \mathrm{~b}_{2}$, $\mathrm{a}_{3}$ e $\mathrm{b}_{3}$ são constantes conhecidas.

Dessa forma, uma densidade a priori conjunta para $\theta$ e $\mathrm{K}$ é dada por,

$$
\pi(\theta, K) \propto \theta^{a_{2}-1} K^{a_{3}-1}(1-K)^{b_{3}-1} e^{-b_{2} \theta} .
$$

\subsubsection{Densidade a Posteriori Conjunta para $\theta$ e K}

Considerando a densidade a priori (3.36) e a função de verossimilhança (2.24), a densidade a posteriori conjunta para $\theta$ e K é dada por,

$$
\pi(\theta, K \mid D) \propto \theta^{n+a_{2}-1} K^{a_{3}+\frac{n(n+1)}{2}-1}(1-K)^{b_{3}-1} \exp \left\{-\left(b_{2}+\sum_{i=1}^{n} K^{i-1} t_{i}\right) \theta\right\}
$$

onde $\theta>0$ e $0 \leq \mathrm{K} \leq 1$.

\subsubsection{Densidades a Posteriori Condicionais para $\theta$ e K}

Através da densidade a posteriori conjunta (3.37), obtemos as densidades condicionais de $\theta$ e K requeridas para o algorítmo Gibbs Sampling, dadas por,

$$
\begin{gathered}
\theta \mid \mathrm{K}, \mathrm{D} \sim \Gamma\left[\mathrm{n}+\mathrm{a}_{2}, \mathrm{~b}_{2}+\sum_{\mathrm{i}=1}^{\mathrm{n}} \mathrm{K}^{\mathrm{i}-1} \mathrm{t}_{\mathrm{i}}\right], \\
\pi(\mathrm{K} \mid \theta, \mathrm{D}) \propto \mathrm{K}^{\mathrm{a}_{3}+\frac{\mathrm{n}(\mathrm{n}+1)}{2}-1}(1-\mathrm{K})^{\mathrm{b}_{3}-1} \exp \left\{-\theta \sum_{\mathrm{i}=1}^{\mathrm{n}} \mathrm{K}^{\mathrm{i}-1} \mathrm{t}_{\mathrm{i}}\right\},
\end{gathered}
$$


onde $\Gamma\left[\mathrm{n}+\mathrm{a}_{2}, \mathrm{~b}_{2}+\sum_{\mathrm{i}=1}^{\mathrm{n}} \mathrm{K}^{\mathrm{i}-1} \mathrm{t}_{\mathrm{i}}\right]$ denota a distribuição Gama e $\mathrm{K}$ pode ser gerado usando o algorítmo Metrópolis Hastings, (ver por exemplo, Chib e Greenberg, 1994).

Dessa forma, a distribuição condicional de $\mathrm{K}$ dado $\theta$ e os dados $\mathrm{D}$ pode ser escrita por,

$$
\pi(\mathrm{K} \mid \theta, \mathrm{D}) \propto \mathrm{K}^{\mathrm{a}_{3}-1}(1-\mathrm{K})^{\mathrm{b}_{3}-1} \psi_{1}(\theta, \mathrm{K}),
$$

onde $\psi_{1}(\theta, K)=\exp \left\{\frac{\mathrm{n}(\mathrm{n}-1)}{2} \ln \mathrm{K}-\theta \sum_{\mathrm{i}=1}^{\mathrm{n}} \mathrm{K}^{\mathrm{i}-1} \mathrm{t}_{\mathrm{i}}\right\}$.

Podemos perceber em (3.39) que a distribuição Beta $\mathrm{B}\left[\mathrm{a}_{3}, \mathrm{~b}_{3}\right]$ define o núcleo de transição do algorítmo, ou seja, os valores de K podem ser simulados tirando na s-ésima iteração (dados os valores correntes de $\theta^{(\mathrm{s})}$ ) um candidato $\mathrm{K}^{(\mathrm{s})}$ da distribuição Beta $\mathrm{B}\left[\mathrm{a}_{3}, \mathrm{~b}_{3}\right]$.Se é satisfeita a estacionariedade, movemos esse ponto com probabilidade,

$$
\min \left\{\frac{\psi_{1}\left(\theta^{(\mathrm{s})}, \mathrm{K}^{(\mathrm{s})}\right)}{\psi_{1}\left(\theta^{(\mathrm{s})}, \mathrm{K}^{(\mathrm{s}-1)}\right)}, 1\right\}
$$

em caso contrário, tomamos $\mathrm{K}^{(\mathrm{s})}=\mathrm{K}^{(\mathrm{s}-1)}$, sendo $\psi_{1}(\theta, \mathrm{K})$ definido em (3.39), (ver apêndice 2).

\subsection{Análise Bayesiana para o Modelo de Goel e Okumoto}

Nesta seção, considerando distribuições a priori informativas para os parâmetros do modelo de Goel e Okumoto, descrevemos uma análise Bayesiana para esse modelo, utilizando o algorítmo Gibbs Sampling com Metrópolis Hastings. 


\subsubsection{Densidades a Priori para $\lambda, \mathrm{N}$ e p}

De acordo com o espaço paramétrico dos parâmetros, consideramos as seguintes densidades a priori para $\lambda, \mathrm{N}$ e p:

$$
\begin{gathered}
\lambda \sim \Gamma\left[\mathrm{a}_{4}, \mathrm{~b}_{4}\right], \\
\mathrm{N} \sim \mathrm{P}\left(\theta_{2}\right), \\
\mathrm{p} \sim \mathrm{B}\left[\mathrm{a}_{5}, \mathrm{~b}_{5}\right],
\end{gathered}
$$

onde $\Gamma\left[\mathrm{a}_{4}, \mathrm{~b}_{4}\right]$ denota a distribuição Gama com média $\mathrm{a}_{4} / \mathrm{b}_{4}, \mathrm{P}\left(\theta_{2}\right)$ denota a distribuição Poisson com média $\theta_{2}, B\left[a_{5}, b_{5}\right]$ denota a distribuição Beta com média $\left[a_{5} /\left(a_{5}+b_{5}\right)\right]$ e $\mathrm{a}_{4}, \mathrm{~b}_{4}, \mathrm{a}_{5}, \mathrm{~b}_{5}$ e $\theta_{2}$ são constantes conhecidas.

Considerando independência entre as distribuições, uma densidade a priori conjunta para $\lambda, \mathrm{N}$ e $\mathrm{p}$, pode ser dada por:

$$
\pi(\lambda, N, p) \propto \frac{\lambda^{a_{4}-1} \theta_{2}^{N}}{N !} \exp \left\{-\left(b_{4} \lambda+\theta_{2}\right)\right\} p^{a_{5}-1}(1-p)^{b_{5}-1}
$$

onde $\lambda>0,0 \leq p \leq 1$ e $N=n, n+1, \ldots$

\subsubsection{Densidade a Posteriori Conjunta para $\lambda, \mathrm{N}$ e p.}

Considerando a função de verossimilhança (2.43) e a densidade a priori conjunta (3.42), a densidade a posteriori conjunta para $\lambda, \mathrm{N}$ e p é dada por,

$$
\begin{aligned}
\pi(\lambda, N, p \mid D) & \propto \frac{\lambda^{n+a_{4}-1} A(N, p) \theta_{2}^{N}}{N !} \times p^{a_{5}-1}(1-p)^{b_{5}-1} \\
& \times \exp \left\{-\left[b_{4}+p \sum_{i=1}^{n} x_{i}+(N-n p) x_{n}\right]\right\} \lambda,
\end{aligned}
$$


onde $A(N, p)=\prod_{i=1}^{n}[N-p(i-1)]$

\subsubsection{Densidades a Posteriori Condicionais para $\lambda, \mathbf{N}$ e p.}

Considerando a densidade a posteriori conjunta (3.42), as densidades condicionais de $\lambda$, N e p, requeridas para o algorítmo Gibbs Sampling, são dadas por,

$$
\begin{gathered}
\lambda \mid N, p, D \sim \Gamma\left[n+a_{4}, b_{4}+p \sum_{i=1}^{n} x_{i}+(N-n p) x_{n}\right], \\
\pi(N \mid / \lambda, p, D) \propto \frac{e^{-\theta_{2}} \theta_{2}^{N}}{N !} \psi_{2}(N, p, \lambda), \\
\pi(p \mid N, \lambda, D) \propto p^{a_{5}-1}(1-p)^{b_{5}-1} \psi_{3}(N, p, \lambda)
\end{gathered}
$$

onde $\psi_{2}(N, p, \lambda)=\exp \left\{\ln A(N, p)-(N-n p) x_{n} \lambda\right\} e$

$$
\psi_{3}(N, p, \lambda)=\exp \left\{\ln A(N, p)-p \lambda \sum_{i=1}^{n} x_{i}-(N-n p) x_{n} \lambda\right\}
$$

Podemos perceber que $\lambda$ dado $N$, p e os dados $\mathrm{D}=\left\{\mathrm{x}_{1}, \ldots, \mathrm{x}_{\mathrm{n}}\right\}$, tem distribuição Gama com média $\left[\left(\mathrm{n}+\mathrm{a}_{4}\right) /\left(\mathrm{b}_{4}+\mathrm{B}(\mathrm{N}, \mathrm{p})\right)\right]$ e pode ser gerado através do algorítmo Gibbs Sampling; $\mathrm{N}$ e p podem ser gerados utilizando o algorítmo Metrópolis Hastings, (ver apêndices 1 e 2).

\subsection{Análise Bayesiana para o Modelo de Schick e Wolverton}

Considerando a função densidade dos tempos entre falhas (2.70) e a função de verossimilhança (2.72) sob o modelo de falha truncada, descrevemos nesta seção, uma análise Bayesiana com o uso do algorítmo Gibbs Sampling com Metrópolis Hastings para o modelo de Schick e Wolverton. 


\subsubsection{Densidades a Priori para $\lambda$ e N.}

Considerando independência entre as distribuições a priori, podemos supor, segundo o espaço paramétrico, as seguintes densidades a priori para $\mathrm{N}$ e $\lambda$ :

$$
\begin{gathered}
\lambda \sim \Gamma\left[\mathrm{a}_{6}, \mathrm{~b}_{6}\right], \\
\mathrm{N} \sim \mathrm{P}\left(\theta_{3}\right),
\end{gathered}
$$

onde $\mathrm{a}_{6}, \mathrm{~b}_{6}$ e $\theta_{3}$ são constantes conhecidas.

Dessa forma, a densidade a priori conjunta para $\lambda$ e $\mathrm{N}$ é dada por:

$$
\pi(\lambda, N) \propto \frac{\lambda^{a_{6}-1} \theta_{3}^{N}}{N !} \exp \left\{-\left(b_{6} \lambda+\theta_{3}\right)\right\}
$$

\subsubsection{Densidade a Posteriori Conjunta para $\lambda$ e $\mathbf{N}$.}

Considerando a função de verossimilhança (2.72) e a densidade a priori conjunta (3.45), obtemos a densidade a posteriori conjunta dada por,

$$
\pi(\lambda, N \mid D) \propto \frac{\lambda^{\mathrm{n}+\mathrm{a}_{6}-1} \mathrm{~A}_{1}(\mathrm{~N}) \theta_{3}^{\mathrm{N}}}{\mathrm{N} !} \exp \left\{-\lambda\left[\mathrm{b}_{6}+\frac{\mathrm{N}}{2} \sum_{\mathrm{i}=1}^{\mathrm{n}} \mathrm{t}_{\mathrm{i}}^{2}-\frac{1}{2} \sum_{\mathrm{i}=1}^{\mathrm{n}}(\mathrm{i}-1) \mathrm{t}_{\mathrm{i}}^{2}\right]\right\},
$$

onde. $\lambda>0$ e $N=n, n+1, \ldots$ 


\subsubsection{Densidades a Posteriori Condicionais para $\lambda$ e $\mathbf{N}$.}

As densidades a posteriori condicionais para o algorítmo Gibbs Sampling, são dadas por,

$$
\lambda \mid \mathrm{N}, \mathrm{D} \sim \Gamma\left[\mathrm{n}+\mathrm{a}_{6}, \mathrm{~b}_{6}+\frac{\mathrm{N}}{2} \sum_{\mathrm{i}=1}^{\mathrm{n}} \mathrm{t}_{\mathrm{i}}^{2}-\frac{1}{2} \sum_{\mathrm{i}=1}^{\mathrm{n}}(\mathrm{i}-1) \mathrm{t}_{\mathrm{i}}^{2}\right],
$$

e

$$
\pi(N \mid \lambda, D) \propto \frac{e^{-\theta_{3}} \theta_{3}^{N}}{N !} A_{1}(N) \exp \left\{-\frac{\lambda}{2} N \sum_{i=1}^{n} t_{i}^{2}\right\} .
$$

Assim como nos casos anteriores, considerando a transformação $\mathrm{N}^{\prime}=\mathrm{N}-\mathrm{n}$ observamos que, $\frac{A_{1}(N)}{N !}=\prod_{i=1}^{n} \frac{t_{i}}{N^{\prime} !} ;$ portanto tem-se que:

$$
\lambda \mid \mathrm{N}^{\prime}, \mathrm{D} \sim \Gamma\left[\mathrm{n}+\mathrm{a}_{6}, \mathrm{~b}_{6}+\frac{\mathrm{N}^{\prime}}{2} \sum_{\mathrm{i}=1}^{\mathrm{n}} \mathrm{t}_{\mathrm{i}}^{2}+\frac{\mathrm{n}}{2} \sum_{\mathrm{i}=1}^{\mathrm{n}} \mathrm{t}_{\mathrm{i}}^{2}-\frac{1}{2} \sum_{\mathrm{i}=1}^{\mathrm{n}}(\mathrm{i}-1) \mathrm{t}_{\mathrm{i}}^{2}\right],
$$

e

$$
\mathrm{N}^{\prime} \mid \lambda, \mathrm{D} \sim \mathrm{P}\left(\theta_{3} \mathrm{e}^{-\lambda \sum_{\mathrm{i}=1}^{\mathrm{n}} \frac{\mathrm{t}_{\mathrm{i}}^{2}}{2}}\right)
$$




\subsection{O Modelo de Littlewood e Verral (1973)}

Littlewood e Verral (1973) introduziram um modelo Bayesiano para a confiabilidade de software, analisando também dados sobre tempos entre falhas do software.

Eles assumem o i-ésimo tempo entre falhas ser exponencial com função densidade de probabilidade (2.2), com taxa de falha $\lambda_{\mathrm{i}}$.

Nesse modelo a sequência de $\lambda_{i}$ 's é assumida estocasticamente decrescente, isto é, $\mathrm{P}\left(\lambda_{\mathrm{i}+1}\right.$ $<\lambda) \geq \mathrm{P}\left(\lambda_{\mathrm{i}}<\lambda\right)$ para $\mathrm{i}=1,2, \ldots$ e $\lambda \geq 0$, em vez de $\lambda_{\mathrm{i}}$ decrescendo com certeza como é assumido no modelo de JM (1972), (veja figura 4 que mostra a taxa de falha para o modelo de LV).

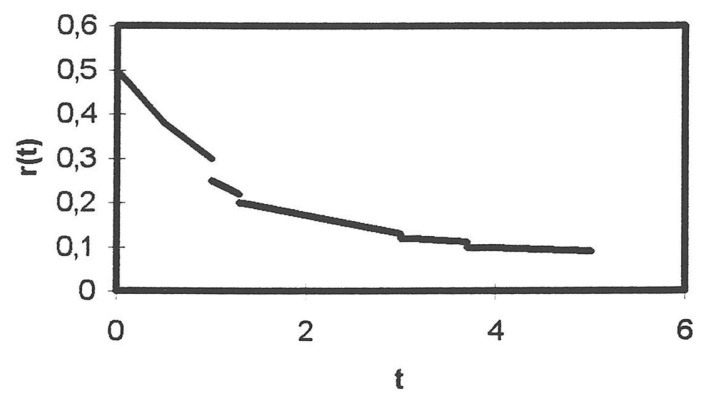

figura 4: Taxa de Falha para o modelo LV

\subsubsection{Formulação do Modelo de Littlewood e Verral (1973)}

Littlewood e Verral consideram uma distribuição Gama para $\lambda_{\mathrm{i}}$ com parâmetro $\alpha$ e escalar $\psi(i)$, onde $\psi(i)$ é uma função de i monotonicamente crescente, isto é,

$$
\pi_{\lambda_{i}}(\lambda \mid \alpha, \psi(i))=\frac{[\psi(i)]^{\alpha}}{\Gamma(\alpha)} \lambda^{\alpha-1} e^{-\psi(i) \lambda}
$$


onde $\lambda \geq 0$.

A escolha da distribuição Gama para a taxa de falha é em grande parte justificada pela sua flexibilidade (tendo dois parâmetros) e sua extensão $(0, \infty)$, além disso ela dá a possibilidade de obter a sequência requerida sobre a distribuição dos $\lambda_{i}$ 's.

A função $\psi(i)$ é suposta descrever a qualidade do programador e a tarefa do programa. Littlewood e Verral especificam $\psi(\mathrm{i})=\beta_{0}+\beta_{1} \mathrm{i}$ que também é assumido por Mazzuchi e Soyer (1988).

Então, assumindo o modelo de Littlewood e Verral, onde o i-ésimo tempo entre falha é assumido ter uma densidade exponencial (2.2) com taxa de falha $\lambda_{\mathrm{i}}$, e uma densidade a priori para $\lambda_{i}$ (3.50), com $\psi(i)=\beta_{0}+\beta_{1}$ i (como considerado por Mazzuchi e Soyer, 1988), a função de verossimilhança para $\alpha, \beta_{0}$ e $\beta_{1}$ é dada por,

$$
\mathrm{L}\left(\alpha, \beta_{0}, \beta_{1}\right)=\prod_{i=1}^{\mathrm{n}} \int_{0}^{\infty} \mathrm{f}\left(\mathrm{t}_{\mathrm{i}} \mid \lambda_{\mathrm{i}}\right) \pi\left[\lambda_{\mathrm{i}} \mid \alpha, \psi(\mathrm{i})\right] \partial \lambda_{\mathrm{i}}
$$

onde $f\left(t_{i} \mid \lambda_{i}\right)=\lambda_{i} e^{-\lambda_{i} t_{i}}$ e $\pi\left[\lambda_{i} \mid \alpha, \psi(i)\right]$ é dada pela expressão (3.50), isto é,

$$
\mathrm{L}\left(\alpha, \beta_{0}, \beta_{1}\right) \propto \alpha^{\mathrm{n}} \exp \left\{-\gamma_{1} \alpha-\gamma_{2}\right\}
$$

onde $\gamma_{1}=\sum_{i=1}^{n} \ln \left(1+\frac{t_{i}}{\psi(i)}\right), \gamma_{2}=\sum_{i=1}^{n} \ln \left(t_{i}+\psi(i)\right)$ e $\psi(i)=\beta_{0}+\beta_{1} i$

\subsubsection{Uma Análise Bayesiana para o Modelo de Littlewood e Verral}

Assumindo o modelo de Littlewood e Verral onde o i-ésimo tempo entre falhas é assumido ter uma densidade exponencial com taxa de falha $\lambda_{i}$, e uma densidade a priori Gama 
para $\lambda_{i} \sim \Gamma[\alpha, \psi(i)]$, com $\psi(i)=\beta_{0}+\beta_{1} i$, consideramos nesta seção, uma análise Bayesiana com o uso do algorítmo de Gibbs Sampling com Metrópolis Hastings.

3.7.3 Densidades a Priori para $\alpha, \beta_{0}$ e $\beta_{1}$.

Assumimos, como Mazzuchi e Soyer (1988), as seguintes densidades a priori para $\alpha, \beta_{0}$ e $\beta_{1}$ :

$$
\begin{gathered}
\alpha \sim \Gamma\left[\mathrm{a}_{7}, \mathrm{~b}_{7}\right], \\
\beta_{1} \sim \Gamma\left[\mathrm{a}_{8}, \mathrm{~b}_{8}\right],
\end{gathered}
$$

e

$$
\pi\left(\beta_{0} \mid \beta_{1}\right) \propto\left(\beta_{0}+\beta_{1}\right)^{a_{9}-1} \exp \left\{-b_{9}\left(\beta_{0}+\beta_{1}\right)\right\},
$$

onde $a_{7}, b_{7}, a_{8}, b_{8}, a_{9}, b_{9}$ são constantes conhecidas baseadas na opinião a priori do especialista.

Assumindo independência a priori, a densidade a priori conjunta para $\alpha, \beta_{0}$ e $\beta_{1}$ é dada por,

$$
\pi\left(\alpha, \beta_{0}, \beta_{1}\right) \propto \alpha^{a_{7}-1} e^{-b_{7} \alpha}\left(\beta_{0}+\beta_{1}\right)^{a_{9}-1} e^{-b_{9}\left(\beta_{0}+\beta_{1}\right)} e^{-b_{8}} \beta_{1}^{a_{8}-1}
$$

\subsubsection{Densidade a Posteriori Conjunta para $\alpha, \beta_{0}$ e $\beta_{1}$}

Considerando a densidade a priori (3.53) e a função de verossimilhança (2.90), a densidade a posteriori conjunta para $\alpha, \beta_{0}$ e $\beta_{1}$ é dada por,

$$
\begin{aligned}
\pi\left(\alpha, \beta_{0}, \beta_{1} \mid D\right) & \propto \alpha^{\mathrm{n}+\mathrm{a}_{7}-1} \mathrm{e}^{-\left[\mathrm{b}_{7}+\gamma_{1}\right] \alpha} \mathrm{e}^{-\gamma_{2}} \times\left(\beta_{0}+\beta_{1}\right)^{\mathrm{a}_{9}-1} \\
& \times \mathrm{e}^{-\left(\beta_{0}+\beta_{1}\right) \mathrm{b}_{9}} \beta_{1}^{\mathrm{a}_{8}-1} \mathrm{e}^{-\mathrm{b}_{8} \beta_{1}}
\end{aligned}
$$


onde $\alpha>0, \beta_{1}>0$ e $\beta_{0}>0$.

3.7.5 Densidades a Posteriori Condicionais para $\alpha, \beta_{0}$ e $\beta_{1}$.

Considerando a densidade a posteriori conjunta (3.54), as densidades a posteriori condicionais para $\alpha, \beta_{0}$ e $\beta_{1}$ requeridas para o algorítmo Gibbs Sampling, são dadas respectivamente por,

$$
\begin{gathered}
\alpha \mid \beta_{0}, \beta_{1} \mathrm{D} \sim \Gamma\left[\mathrm{n}+\mathrm{a}_{7}, \gamma_{1}+\mathrm{b}_{7}\right], \\
\pi\left(\beta_{0} \mid \alpha, \beta_{1}, \mathrm{D}\right) \propto\left(\beta_{0}+\beta_{1}\right)^{\mathrm{a}_{9}-1} \mathrm{e}^{-\left(\beta_{0}+\beta_{1}\right) \mathrm{b}_{9}} \psi_{4}\left(\alpha, \beta_{0}, \beta_{1}\right), \\
\pi\left(\beta_{1} \mid \alpha, \beta_{0}, \mathrm{D}\right) \propto \beta_{1}^{\mathrm{a}_{8}-1} \mathrm{e}^{-\mathrm{b}_{8} \beta_{1}} \psi_{5}\left(\alpha, \beta_{0}, \beta_{1}\right),
\end{gathered}
$$

onde, $\psi_{4}\left(\alpha, \beta_{0}, \beta_{1}\right)=\exp \left\{-\gamma_{1} \alpha-\gamma_{2}\right\}$ e

$\psi_{5}\left(\alpha, \beta_{0}, \beta_{1}\right)=\exp \left\{\left(a_{9}-1\right) \ln \left(\beta_{0}+\beta_{1}\right)-b_{9} \beta_{1}-\gamma_{1} \alpha-\gamma_{2}\right\}$

De (3.55) podemos observar que $\beta_{0}$ e $\beta_{1}$ podem ser gerados utilizando o algorítmo Metrópolis Hasting e $\alpha$ pode ser gerado através do algorítmo Gibbs Sampling. 
Capítulo 4

\section{ALGUMAS CONSIDERAÇÕES SOBRE SELEÇÃO DE MODELOS}

Nos capítulos anteriores foram apresentados diversos modelos de confiabilidade de software enfatizando a estratégia tipo I (estratégia de modelos que modelam os tempos entre falhas do software).

Uma questão que surge naturalmente é a seguinte: Qual ou quais modelos são mais apropriados para análise dos tempos entre falhas do software?

Neste sentido, a metodologia para determinação de modelos se torna fundamental. Ela se divide em duas componentes: adequação do modelo e seleção do modelo.

Neste trabalho nos preocupamos em selecionar o melhor modelo dentre a coleção de modelos estudados. A discussão relativa à escolha de modelos enfatiza as distribuições preditivas como elemento principal na comparação de modelos arbitrários.

Sob o enfoque Bayesiano, a seleção de modelos pode ser conduzida através de muitas estratégias propostas na literatura (ver por exemplo, Jeffreys, 1939; Box e Hill, 1967; Geisser e Edy, 1979; Gelfand e Dey, 1994; e Gelfand, Dey e Chang 1992).

Destacamos nesse trabalho, a técnica das distribuições preditivas condicionais ordenadas (CPO). Na seção 4.1 a densidade preditiva condicional ordenada (CPO) é descrita, enfatizando na seção 4.2 o uso do método de Monte Carlo para estimar as densidades preditivas em questão e na seção 4.3 delineamos a técnica para selecionar o melhor modelo.

\subsection{Distribuições Preditivas}

Em geral, supomos que um modelo estatístico paramétrico é definido pela especificação da distribuição conjunta para as observações $\mathrm{Y}=\left\{\mathrm{y}_{1}, \ldots, \mathrm{y}_{\mathrm{n}}\right\}$ e para os parâmetros do modelo, $\theta=\left\{\theta_{1}, \theta_{2}, \ldots, \theta_{\mathrm{p}}\right\}$ onde $\mathrm{Y}_{\mathrm{r}}, \mathrm{r}=1,2, \ldots, \mathrm{n}$ é conhecido. 
Portanto, considere $\mathrm{f}(\mathrm{Y} \mid \theta)$ (representando a densidade conjunta das observações dado $\theta$ ) e $\pi(\theta)$ (a densidade a priori de $\theta$ ) tendo $f(Y \mid \theta) \pi(\theta)$ como especificação do modelo. Se $\mathrm{Y}$ e $\theta$ são supostos contínuos, podemos notar em geral, que a densidade preditiva pode ser definida por

$$
f(Y)=\int f(Y \mid \theta) \pi(\theta) d \theta
$$

A distribuição preditiva (ou verossimilhança marginal), é a distribuição marginal conjunta dos dados, que pode ser utilizada de várias formas na determinação de modelos.

Podemos notar que, caso a distribuição a priori $\pi(\theta)$ seja própria, não há nenhum problema na utilização de $\mathrm{f}(\mathrm{Y})$ na seleção de modelos, mas caso a densidade a priori seja imprópria, $\mathrm{f}(\mathrm{Y})$ não pode ser calculada pois a integral pode divergir.

Neste sentido, outras densidades foram analisadas e sob diferentes justificativas podem ser usadas na discriminação de modelos.

Uma densidade preditiva que foi utilizada extensivamente em trabalhos recentes de Gelfand (1995) e Gelfand, Dey e Chang (1992) é a densidade preditiva "cross-validation" dada por,

$$
f\left(Y_{r} \mid Y_{(r)}\right)=\frac{f(Y)}{f\left(Y_{(r)}\right)}=\int f\left(Y_{r} \mid \theta, Y_{(r)}\right) \pi\left(\theta \mid Y_{(r)}\right) d \theta
$$

onde $\mathrm{Y}_{(\mathrm{r})}=\left(\mathrm{Y}_{1}, \ldots, \mathrm{Y}_{\mathrm{r}-1}, \mathrm{Y}_{\mathrm{r}+1}, \ldots, \mathrm{Y}_{\mathrm{n}}\right)$ e $\pi\left(\theta \mid \mathrm{Y}_{(\mathrm{r})}\right)$ é a densidade a posteriori de $\theta$. Essa densidade pode ser chamada de densidade preditiva condicional ordenada (CPO).

A aproximação "cross-validation", um único ponto deletado, é uma rotina padrão em análise de regressão clássica e muitos diagnósticos adotam esse ponto de vista. Isto também é bem estabelecido na literatura Bayesiana por Stone (1974) e Geisser (1975). 
Considerando os tempos entre falhas do software $\left\{t_{1}, \ldots, t_{n}\right\}$, utilizamos a densidade de $t_{i}$ dado $D_{(i)}, i=1, \ldots, n$ onde $D_{(i)}$ denota os tempos entre falhas do software não incluindo $t_{i}$, isto é, $D_{(i)}=\left\{t_{1}, \ldots, t_{i-1}, t_{i+1}, \ldots, t_{n}\right\}$.

Dessa forma, a densidade preditiva de $t_{i}$ dado $D_{(i)}$ para o modelo de Jelinski e Moranda (2.1) pode ser dada por,

$$
c_{i}=f\left(t_{i} \mid D_{(i)}\right)=\sum_{N^{\prime}=0}^{\infty} \int_{0}^{\infty} f\left(t_{i} \mid N^{\prime}, \lambda\right) \pi\left(N^{\prime}, \lambda \mid D_{(i)}\right) d \lambda
$$

Similarmente, definimos as densidades preditivas de $t_{i}$ dado $D_{(i)}, i=1, \ldots, n$ para os modelos de Moranda (2.22), Goel e Okumoto (2.41) e Schick e Wolverton (2.69).

Considerando o modelo de Littlewood e Verral (1973), a densidade preditiva de $t_{i}$ dado $\mathrm{D}_{(\mathrm{i})}$ é dada por,

$$
c_{i}=f\left(t_{i} \mid D_{(i)}\right)=\int_{0}^{\infty} f\left(t_{i} \mid \lambda_{(i)}\right) \pi\left(\lambda_{i} \mid D_{(i)}\right) d \lambda_{i}
$$

isto é,

$$
\mathrm{f}\left(\mathrm{t}_{\mathrm{i}} \mid \mathrm{D}_{(\mathrm{i})}\right)=\int_{0}^{\infty} \lambda_{\mathrm{i}} \mathrm{e}^{-\lambda_{\mathrm{i}} \mathrm{t}_{\mathrm{i}}}\left\{\iiint \pi\left(\lambda_{\mathrm{i}} \mid \alpha, \psi(\mathrm{i})\right) \pi\left(\alpha, \beta_{0}, \beta_{1} \mid \mathrm{D}_{(\mathrm{i})}\right) \partial \alpha \partial \beta_{0} \partial \beta_{1}\right\} \partial \lambda_{\mathrm{i}}
$$

onde $\lambda_{i} \mid \alpha, \psi(i) \sim \Gamma[\alpha, \psi(i)]$ e $\psi(i)=\beta_{0}+\beta_{1} i$

Considerando a ordem de integração, temos: 


$$
\mathrm{f}\left(\mathrm{t}_{\mathrm{i}} \mid \mathrm{D}_{(\mathrm{i})}\right)=\iiint \frac{\alpha\left[\beta_{0}+\beta_{1} \mathrm{i}\right]^{\alpha}}{\left(\mathrm{t}_{\mathrm{i}}+\beta_{0}+\beta_{1} \mathrm{i}\right)^{\alpha+1}} \pi\left(\alpha, \beta_{0}, \beta_{1} \mid \mathrm{D}_{(\mathrm{i})}\right) \partial \alpha \partial \beta_{0} \partial \beta_{1}
$$

Podemos notar que as integrais da equações (4.1) e (4.5) são analiticamente difíceis de se resolver e necessitam de integração numérica. Uma alternativa é utilizar o método de integração de Monte Carlo, (ver por exemplo, Kloek e Van Dijk, 1978).

\subsection{Estimativas de Monte Carlo para CPO's}

Considerando a densidade preditiva "cross-validation", descrita em (4.2), podemos notar que uma estimativa da densidade preditiva requer somente o valor de $\theta$, isto é,

$$
f\left(Y_{r} \mid Y_{(r)}\right)=\frac{f(Y)}{f\left(Y_{(r)}\right)}=\frac{\int \frac{f(Y \mid \theta) \pi(\theta)}{\pi(\theta \mid Y) f(Y)} \pi(\theta / Y) d \theta}{\int \frac{f\left(Y_{(r)} \mid \theta\right) \pi(\theta)}{\pi\left(\theta \mid Y_{(r)}\right) f(Y)} \pi(\theta / Y) d}=\frac{1}{\int \frac{1}{f\left(Y_{r} \mid Y_{(r)}, \theta\right)} \pi(\theta / Y) d \theta}
$$

Usando o algorítmo Gibbs Sampling (ver apêndice), é possível (como mencionado no capítulo 3), gerar observações da distribuição $\pi(\theta / Y)$, que podem ser utilizadas nos cálculos da distribuição preditiva condicional ordenada (CPO), a qual estamos interessados. Portanto uma imediata integração de Monte Carlo nos dá:

$$
\hat{\mathrm{f}}\left(\mathrm{Y}_{\mathrm{r}} \mid \mathrm{Y}_{(\mathrm{r})}\right)=\mathrm{S}\left(\sum_{\mathrm{s}=1}^{\mathrm{S}} \frac{1}{\mathrm{f}\left(\mathrm{y}_{\mathrm{r}} / \mathrm{Y}_{(\mathrm{r})}, \theta^{(\mathrm{s})}\right)}\right)^{-1}
$$


que pode ser referida como uma estimativa $\mathrm{CPO}$, onde $\theta^{(\mathrm{s})}$ é gerado pelo algorítmo Gibbs Sampling por S iterações consideradas.

Neste sentido, usando as amostras geradas pelo algorítmo Gibbs Sampling, (4.3) pode ser aproximado por um estimador de Monte Carlo dado por,

$$
=\hat{f}\left(t_{i} \mid D_{(i)}\right)=\frac{2}{R S} \sum_{i=1 S=\frac{S}{2}+1}^{R} \sum_{S}^{S} \lambda^{(r, s)}\left(N^{\prime(r, s)}+n-i+1\right) e^{-\lambda^{(r, s)}\left(N^{\prime(r, s)}+n-i+1\right) t_{i}}
$$

onde $\mathrm{i}=1,2, \ldots, \mathrm{n} ; \lambda^{(\mathrm{r}, \mathrm{s})}$ e $\mathrm{N}^{(\mathrm{r}, \mathrm{s})}$ são gerados por (3.1) de $\mathrm{S}$ iterações para cada uma das $\mathrm{R}$ cadeias considerando diferentes valores iniciais para $\lambda$ e $\mathrm{N}^{\prime}$.

Da mesma forma, utilizando as amostras geradas pelo algorítmo Gibbs Sampling, obtemos estimativas de Monte Carlo para as densidades preditivas de $\mathrm{t}_{\mathrm{i}}, \mathrm{i}=1, \ldots, \mathrm{n}$ dado $\mathrm{D}_{(\mathrm{i})}$ para os modelos de Moranda, Goel e Okumoto e Schick e Wolverton.

Para o modelo de Littlewood e Verral, um estimador de Monte Carlo para $c_{i}=f\left(t_{i} \mid D_{(i)}\right)$ baseado nas amostras geradas pelo algorítmo Gibs Sampling é dado por,

$$
\hat{\mathrm{f}}\left(\mathrm{t}_{\mathrm{i}} \mid \mathrm{D}_{(\mathrm{i})}\right)=\frac{2}{\mathrm{RS}} \sum_{\mathrm{i}=1 \mathrm{~S}=\frac{\mathrm{s}}{2}+1}^{\mathrm{S}} \frac{\alpha^{(\mathrm{r}, \mathrm{s})}\left(\beta_{0}^{(\mathrm{r}, \mathrm{s})}+\beta_{1}^{(\mathrm{r}, \mathrm{s})} \mathrm{i}\right)^{\alpha(\mathrm{r}, \mathrm{s})}}{\left(\mathrm{t}_{\mathrm{i}}+\beta_{0}^{(\mathrm{r}, \mathrm{s})}+\beta_{1}^{(\mathrm{r}, \mathrm{s})_{i}}\right)^{\alpha(\mathrm{r}, \mathrm{s})+1}}
$$

onde $\mathrm{i}=1,2, \ldots, \mathrm{n}$ para $\mathrm{S}$ iterações em cada $\mathrm{R}$ simulação.

\subsection{Escolha do Modelo}

Podemos utilizar a densidade preditiva $c_{i}=f\left(t_{i} \mid D_{(i)}\right)$ na seleção de modelos, examinando CPO's dos diferentes modelos estudados, observando que o melhor modelo deve ter a grande parte dos valores da CPO maiores do que os valores das CPO's dos modelos alternativos. 
Dessa forma, um gráfico dos valores da $\mathrm{CPO}\left(\mathrm{c}_{\mathrm{i}}\right)$ contra o número de observações $(\mathrm{i}=1$, $2, \ldots, \mathrm{n}$ ) deve revelar que o melhor modelo é aquele que apresenta a maioria dos CPO's acima dos CPO's dos modelos comparados.

Alternativamente, podemos escolher o melhor modelo que maximiza $c(1)=\prod_{i=1}^{n} c_{i}(1)$, onde lé o indexador dos modelos.

Além da técnica acima proposta, poderíamos usar as diferentes versões do fator de Bayes para discriminação de modelos (ver por exemplo, Jeffreys, 1961; Berger e Pericchi, 1993; Aitkin, 1991). 


\section{Capítulo 5}

\section{UM EXEMPLO DE APLICAÇÃO}

Após a introdução nos capítulos anteriores de uma metodologia de análise para os tempos entre falhas do software, é de grande interesse, a título de ilustração, desenvolvermos um exemplo de aplicação das técnicas utilizadas.

Neste exemplo de aplicação, nosso objetivo é a comparação das análises Clássica e Bayesiana, utilizando o algorítmo Gibbs Sampling com Metrópolis Hastings para os modelos de Estratégia tipo I-1, desenvolvidas nos capítulos 2 e 3, e através da metodologia descrita no capítulo 4 , selecionar o melhor modelo de confiabilidade de software considerando os tempos entre falhas.

Utilizamos para essas análises, os dados de um Sistema Naval Americano, introduzidos por Jelinski e Moranda (1972), descritos na tabela 5.1.

Esses dados consistem dos números de dias entre 26 falhas que ocorreram durante a fase de produção de um software (NTDS dados - Naval Tactical Data System).

\begin{tabular}{|c|c|c|c|c|c|c|c|c|}
\hline $\mathrm{i}$ & $\mathrm{t}_{\mathrm{i}}$ & $\mathrm{x}_{\mathrm{i}}$ & $\mathrm{i}$ & $\mathrm{t}_{\mathrm{i}}$ & $\mathrm{x}_{\mathrm{i}}$ & $\mathrm{i}$ & $\mathrm{t}_{\mathrm{i}}$ & $\mathrm{x}_{\mathrm{i}}$ \\
\hline \hline 1 & 9 & 9 & 10 & 7 & 70 & 19 & 6 & 104 \\
2 & 12 & 21 & 11 & 1 & 71 & 20 & 1 & 105 \\
3 & 11 & 32 & 12 & 6 & 77 & 21 & 11 & 116 \\
4 & 4 & 36 & 13 & 1 & 78 & 22 & 33 & 149 \\
5 & 7 & 43 & 14 & 9 & 87 & 23 & 7 & 156 \\
6 & 2 & 45 & 15 & 4 & 91 & 24 & 81 & 247 \\
7 & 5 & 50 & 16 & 1 & 92 & 25 & 2 & 249 \\
8 & 8 & 58 & 17 & 3 & 95 & 26 & 1 & 250 \\
9 & 5 & 63 & 18 & 3 & 98 & & & \\
\hline
\end{tabular}

Tabela 5.1: Dados NTDS $\left(t_{i}=x_{i}-x_{i-1}\right)$

onde $\mathrm{n}=26 \mathrm{e} \mathrm{x}_{\mathrm{n}}=\mathrm{x}_{26}=250$. 


\subsection{Análise Clássica para os Dados}

Nesta seção, considerando os dados da tabela 5.1, aplicamos a metodologia de Análise Clássica baseada na aproximação Normal assintótica dos estimadores de máxima verossimilhança dos parâmetros envolvidos nos modelos de Jelinski e Moranda (1972), Moranda (1975), Goel e Okumoto (1978) e Schick e Wolverton (1978), (ver capítulo 2).

O efeito de uma reparametrização no modelo de Jelinski e Moranda é explorada, considerando métodos para verificação da normalidade da função de verossimilhança dos parâmetros do modelo.

\subsubsection{Análise Clássica dos dados NTDS sob o modelo de Jelinski e Moranda}

Considerando os dados da Tabela 5.1 e as expressões (2.9) e (2.10), obtemos numéricamente os estimadores de máxima verossimilhança para $\lambda$ e $\mathrm{N}$, dados por,

$$
\left\{\begin{array}{l}
\hat{\lambda}=0.00685 \\
\hat{N}=31.2159
\end{array},\right.
$$

onde $\hat{\mathrm{N}}$ (estimador de máxima verossimilhança para $\mathrm{N}$ ) é calculado com o uso do método iterativo Newton - Raphson.

Considerando os estimadores de máxima verossimilhança $\hat{\lambda}$ e $\hat{\mathrm{N}}$, a matriz de variâncias e covariâncias assintótica de $\hat{\lambda}$ e $\hat{N}$, a inversa da matriz de informação de Fisher (2.18), é dada por,

$$
I^{-1}(\hat{\lambda}, \hat{N})=\left[\begin{array}{ll}
0.00001 & -0.01493 \\
-0.01493 & 33.09956
\end{array}\right] \text {. }
$$


Intervalos de confiança $95 \%$ aproximados para $\lambda$ e $N$, utilizando a aproximação assintótica (2.11) dos estimadores de máxima verossimilhança $\hat{\lambda}$ e $\hat{\mathrm{N}}$, são dados por $(0.00065<$ $\lambda<0.01305)$ e $(19.93959<\mathrm{N}<42.4922)$.

\subsubsection{O Efeito de uma Reparametrização para o modelo de Jelinski e Moranda}

Quando procuramos fazer inferências sobre funções de parâmetros, geralmente não encontramos resultados exatos, sendo comum o uso da Normalidade Assintótica do Estimador de Máxima Verossimilhança, baseado na informação de Fisher ou informação observada (ver por exemplo, Bickel e Doksum, 1977).

Esses resultados podem não ser precisos quando trabalhamos com amostras pequenas ou moderadas. Nesse contexto, uma alternativa para melhorar esses resultados, é considerar reparametrizações que melhorem a normalidade da função de verossimilhança (ver por exemplo, Anscombe, 1964 ou Sprott, 1973).

Também na inferência Bayesiana, é importante uma boa parametrização para se obter resultados mais precisos quando utilizamos métodos numéricos ou de aproximação no cálculo de momentos a posteriori, (ver por exemplo, Achcar e Smith, 1990; Kass e Slate, 1992 ou Hills e Smith, 1993).

Assim, para melhorar a normalidade do estimador de máxima verossimilhança no modelo de Jelinski e Moranda, uma possível e usual transformação é dada por $\phi=\ln (\lambda)$ e $N=N$.

Um modo simples de se verificar a normalidade da função de verossimilhança ou densidades a posteriori de interesse, é através de gráficos e contornos (no caso bivariado), onde concluímos pela normalidade quando encontramos formas de eclipses aproximadas.

Em situações quando temos interesse num dado parâmetro e existem vários parâmetros auxiliares (nuisance), podemos (como uma alternativa) considerar a verossimilhança "profile" e verificar sua normalidade através de gráficos. 


\subsubsection{Inferências para a Taxa de Falha considerando a Função de Verossimilhança Profile}

Com $\mathrm{N}$ desconhecido, as inferências sobre a taxa de falha são mais complexas, uma vez que devemos estudar a normalidade conjunta dos dois parâmetros do modelo.

Como alternativa, podemos considerar a função de verossimilhança "profile" de $\lambda$ dada por $\mathrm{L}_{\hat{\mathrm{N}}}(\lambda)=\mathrm{L}(\lambda, \hat{\mathrm{N}})$, onde $\hat{\mathrm{N}}$ maximiza a função de verossimilhança conjunta $\mathrm{L}(\lambda, N)$ para cada valor de $\lambda$. Desta forma, o logarítmo da função de verossimilhança "profile" é dada por,

$$
1_{\hat{N}}(\lambda)=n \ln \lambda+\sum_{i=1}^{n} \ln (\hat{N}-i+1)-\lambda \sum_{i=1}^{n}(\hat{N}-i+1) t_{i},
$$

onde para cada valor de $\lambda, \hat{\mathrm{N}}$ é obtido de:

$$
\sum_{i=1}^{n}\left[\frac{1}{(\hat{N}-i+1)}\right]-\lambda \sum_{i=1}^{n} t_{i}=0
$$

através do método iterativo de Newton - Raphson.

Considerando a transformação $\phi=\ln (\lambda)$, o logarítmo da verossimilhança "profile" é dado por,

$$
1_{\hat{\mathrm{N}}}(\phi)=\mathrm{n} \phi+\sum_{\mathrm{i}=1}^{\mathrm{n}} \ln (\hat{\mathrm{N}}-\mathrm{i}+1)-\mathrm{e}^{\phi} \sum_{\mathrm{i}=1}^{\mathrm{n}}(\hat{\mathrm{N}}-\mathrm{i}+1) \mathrm{t}_{\mathrm{i}}
$$

onde para cada valor de $\phi, \hat{\mathrm{N}}$ é obtido de,

$$
\sum_{i=1}^{n} \frac{1}{\hat{N}-i+1}-e^{\phi} \sum_{i=1}^{n} t_{i}=0
$$


através do método iterativo de Newton - Raphson.

\subsubsection{T-plot de Hills e Smith}

Um método gráfico muito prático para se verificar a normalidade da verossimilhança é dado pelo T-plot introduzido por Hills e Smith,1993.

Em nosso caso, utilizamos o T-plot de Hills e Smith para a função de verossimilhança "profile" $\mathrm{L}_{\hat{\mathrm{N}}}(\lambda)$, a partir da relação:

$$
\begin{aligned}
T(\lambda) & =\operatorname{sgn}(\lambda-\hat{\lambda})\left\{-2 \log \left[\frac{\mathrm{L}_{\hat{\mathrm{N}}}(\lambda)}{\mathrm{L}(\hat{\mathrm{N}}, \hat{\lambda})}\right]\right\}^{1 / 2}= \\
& =\operatorname{sgn}(\lambda-\hat{\lambda})\left\{-2\left[1_{\hat{\mathrm{N}}}(\lambda)-1(\hat{\mathrm{N}}, \hat{\lambda})\right]\right\}^{1 / 2},
\end{aligned}
$$

onde $1_{\hat{N}}(\lambda)$ é o logarítmo da verossimilhança "profile" e $\operatorname{sgn}(\lambda-\hat{\lambda})$ é a função sinal. Dessa forma, considerando os dados NTDS, apresentamos nas figura 5.1 e 5.2 o T-plot e o gráfico da verossimilhança "profile" na parametrização original.

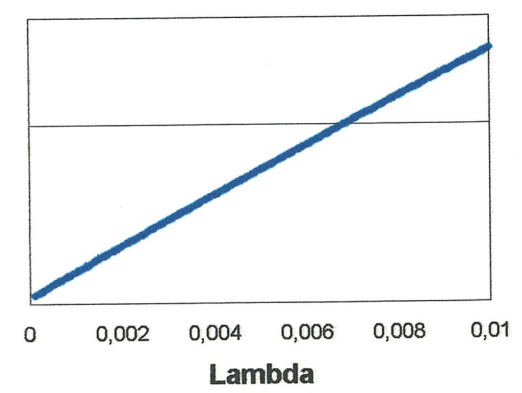

Figura 5.1: T-plot da Verossimilhança "Profile" $T_{\hat{N}}(\lambda)$ 


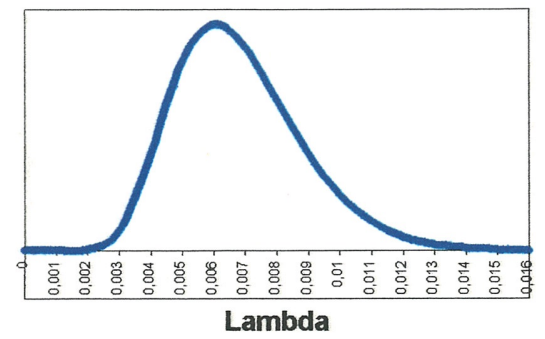

Figura 5.2: Verossimilhança "profile $L_{\hat{N}}(\lambda)$

Conclusão: Observamos que o gráfico T-plot para a parametrização original $\left(\mathrm{T}_{\hat{\mathrm{N}}}(\hat{\lambda})\right)$, apresenta uma boa linearidade, o que nos indica a normalidade da verossimilhança "profile" $\mathrm{L}_{\hat{\mathrm{N}}}(\lambda)$, ou seja, o uso de uma reparametrização torna-se desnecessária segundo a medida de normalidade de Hills e Smith (1993).

\subsubsection{Terceira Derivada Padronizada do Logarítmo da Verossimilhança.}

Kass e Slate (1992) propuseram uma medida de não-normalidade de densidades a posteriori ou funções de verossimilhança, dada pela terceira derivada padronizada (STD), que simplifica as interpretações pois reduz as análises a um simples escalar. A terceira derivada padronizada do logarítmo da verossimilhança em relação a taxa de falha $\lambda$,é dada por,

$$
\operatorname{STD}(\hat{\lambda})=\left|1^{\prime \prime \prime}(\hat{\lambda})\left[-1^{\prime \prime}(\hat{\lambda})\right]^{-3 / 2}\right|,
$$

onde $1^{\prime \prime}(\hat{\lambda})$ e $1^{\prime \prime \prime}(\hat{\lambda})$ são respectivamente, a segunda e a terceira derivadas do logarítmo da função de verossimilhança calculado no estimador de máxima verossimilhança $\hat{\lambda}$. 
Se a função de verossimilhança apresentar "boa normalidade", então esperamos encontrar $\operatorname{STD}(\hat{\lambda})$ próximo ou igual a zero.

Portanto, considerando a função log-verossimilhança (2.6), calculada no ponto de máxima verossimilhança $\hat{\lambda}$, temos que a terceira derivada padronizada é dada por,

$$
\operatorname{STD}(\hat{\lambda})=\left|\frac{2 \mathrm{~B}(\mathrm{~N})^{3}}{\mathrm{n}^{2}}\left[\frac{\mathrm{B}(\mathrm{N})^{2}}{\mathrm{n}}\right]^{-3 / 2}\right|
$$

onde $\mathrm{B}(\mathrm{N})=\sum_{\mathrm{i}=1}^{\mathrm{n}}(\mathrm{N}-\mathrm{i}+1) \mathrm{t}_{\mathrm{i}}$ e $\mathrm{N}$ é suposto conhecido.

Considerando a função log-verossimilhança $(1(\phi, N))$, dada por,

$$
1(\phi, N)=n \phi+\sum_{i=1}^{n} \ln (N-i+1)-e^{\phi} \sum_{i=1}^{n}(N-i+1) t_{i}
$$

a terceira derivada padronizada, calculada no ponto de máxima verossimilhança $\hat{\phi}$, é dada por,

$$
\operatorname{STD}(\hat{\phi})=\left|-\mathrm{n}[\mathrm{n}]^{-3 / 2}\right|
$$

Com os dados NTDS, descritos na tabela 5.1, onde $n=26$ e $N=31$ conhecido, $\operatorname{STD}(\hat{\lambda})$ $=0.39223$ e $\operatorname{STD}(\hat{\phi})=0.19612$.

Conclusão: Através da medida de não-normalidade introduzida por Kass e Slate, baseada na terceira derivada padronizada (STD), observamos que utilizando ou não a reparametrização considerada, temos uma "boa normalidade", dado os valores $\operatorname{STD}(\hat{\lambda})$ e $\operatorname{STD}(\hat{\phi})$ relativamente próximos a zero.

Neste caso, não é tão plausível admitirmos que a transformação ou reparametrização $\phi=\ln \lambda$ melhorou a normalidade da função de verossimilhança na parametrização original, dado que as medidas de não-normalidade, $\operatorname{STD}(\hat{\lambda})$ e $\operatorname{STD}(\hat{\phi})$, utilizando os dados da tabela 1 , estão relativamente próximas. 


\subsubsection{Análise Clássica dos dados NTDS sob o Modelo de Moranda}

Considerando os dados da tabela 5.1 e as expressões (2.28) e (2.29), os estimadores de máxima verossimilhança para $\theta$ e $\mathrm{K}$, são dados por, $\hat{\theta}=0.251633$ e $\hat{\mathrm{K}}=0.936947$.

A matriz de variâncias e covariâncias assintóticas de $\hat{\theta}$ e $\hat{\mathrm{K}}$, o inverso da matriz de informação de Fisher (2.37), é dada por,

$$
\mathrm{I}^{-1}(\hat{\theta}, \hat{\mathrm{K}})=\left[\begin{array}{cc}
0920022 & -0.00201510 \\
-0.00201510 & 0.00600025
\end{array}\right]
$$

Considerando a aproximação assintótica (2.30) dos estimadores de máxima verossimilhança $\hat{\lambda}$ e $\hat{\mathrm{N}}$, intervalos de confiança $95 \%$ para $\theta$ e $\mathrm{K}$, são dados por, $(0.6363<\theta<$ $0.43963)$ e $(0.88893<\mathrm{K}<0.98497)$.

\subsubsection{Análise Clássica dos Dados NTDS sob o modelo de Schick e Wolverton}

Considerando os dados da tabela (5.1) e as expressões (2.76) e (2.77), os estimadores de máxima verossimilhança para $\lambda$ e $\mathrm{N}$, são dados por,

$$
\left\{\begin{array}{l}
\hat{\lambda}=0.0013 \\
\hat{N}=25.3585
\end{array},\right.
$$

onde $\hat{\mathrm{N}}$ é obtido através do método de Newton Raphson.

Com esses estimadores, de (2.82) e (2.83) temos que a matriz de informação observada de $\lambda$ e $\mathrm{N}$ é dada por, 


$$
I_{o}(\lambda, N)=\left[\begin{array}{cc}
15384616 & 5157 \\
5157 & 8.8101
\end{array}\right]
$$

Dessa forma, considerando a inversa da matriz de informação observada, intervalos com $95 \%$ de confiança podem ser obtidos, dados por, $(0.00068<\lambda<0.00192)$ e $(24.62196<\mathrm{N}<26.09504)$.

\subsection{Uma Solução Bayesiana na Análise dos Dados NTDS}

Nesta seção, considerando os dados introduzidos por Jelinski e Moranda (tabela 1), aplicamos a metodologia de análise Bayesiana utilizando o algorítmo Gibbs Sampling com Metrópolis Hastings, descrita no capítulo 3, para os principais modelos de estratégia tipo I-1.

\subsubsection{Análise Bayesiana para os Dados NTDS sob o Modelo de Jelinski e Moranda}

\section{1 - Assumindo $\lambda$ com Priori Gama e N com Priori Poisson.}

Assumindo o modelo de falha truncada com $\mathrm{x}_{26}=250$, sob o modelo de Jelinski e Moranda (2.1), consideramos as densidades a priori, descritas em (3.1), para os parâmetros $\lambda$ e $N$, onde $a_{1}, b_{1}$ e $\theta_{1}$ são constantes conhecidas baseadas na informação contida nos dados, por ser inviável obter informações sobre um software desenvolvido em 1972.

Nesse contexto, considerando os estimadores de máxima verossimilhança para $\lambda$ e $N$, que expressam toda a informação contida nos dados e suas respectivas variâncias assintóticas, assumimos $\mathrm{a}_{1}=685, \mathrm{~b}_{1}=5$ a partir do sistema de equações $\left\{\begin{array}{l}\mathrm{E}(\lambda)=\mathrm{a} / \mathrm{b}=\hat{\lambda} \\ \mathrm{V}(\lambda)=\mathrm{a} / \mathrm{b}^{2}=\hat{\sigma}_{\lambda}\end{array}\right\}$ e $\theta=30 \mathrm{a}$ partir do conceito que $E(N)=\theta=\hat{N}$. Dessa forma, as densidades a priori para $\lambda$ e $N$, considerando os dados da tabela 1, são dadas por, 


$$
\begin{gathered}
\lambda \sim \Gamma[5,685], \\
\mathrm{N} \sim \mathrm{P}(30) .
\end{gathered}
$$

De (3.8), as distribuições condicionais de $\left(\lambda / \mathrm{N}^{\prime}, \mathrm{D}\right)$ e $\left(\mathrm{N}^{\prime} / \lambda, \mathrm{D}\right)$, requeridas como funções geradoras no algorítmo Gibbs Sampling, são dadas por,

$$
\lambda / \mathrm{N}^{\prime}, \mathrm{D} \sim \Gamma\left[711,5+250 \mathrm{~N}^{\prime}+\sum_{\mathrm{i}=1}^{26} \mathrm{x}_{\mathrm{i}}\right]
$$

e

$$
\mathrm{N}^{\prime} / \lambda, \mathrm{D} \sim \mathrm{P}\left(30 \mathrm{e}^{-250 \lambda}\right)
$$

onde $\mathrm{N}^{\prime}=\mathrm{N}-26, \sum_{\mathrm{i}=1}^{\mathrm{n}} \mathrm{x}_{\mathrm{i}}=2492$ e $\mathrm{D}$ denota o conjunto de dados sobre tempos de falha $\left\{\mathrm{x}_{1}, \ldots, \mathrm{x}_{26}\right\}$.

Selecão da Amostra: Considerando as distribuições condicionais requeridas para o algorítmo Gibbs Sampling, foram geradas cinco cadeias com 2500 iterações cada, assumindo-se diferentes valores iniciais para as cadeias baseadas em uma análise preliminar dos dados e nos estimadores de máxima verossimilhança.

Para cada parâmetro foram selecionados as $1005^{\text {th }}, 1010^{\text {th }}, \ldots, 2495^{\text {th }}, 2500^{\text {th }}$ iterações, que de cinco cadeias produziu uma amostra de tamanho 1500.

À medida que o número de iterações aumenta, a cadeia se aproxima de sua condição de equilibrío. Assim assume-se que a convergência é atingida nas últimas iterações da cadeia. Nesse sentido, em toda a análise decorrente, os valores iniciais das amostras geradas pelos algorítmos de simulação, são desprezados.

Isso pode ser verificado visualmente através das figuras 5.3 e 5.4, que mostram as trajetórias das amostras de $\mathrm{N}^{\prime}$ e $\lambda$, obtidas pelo algorítmo Gibbs Sampling. 


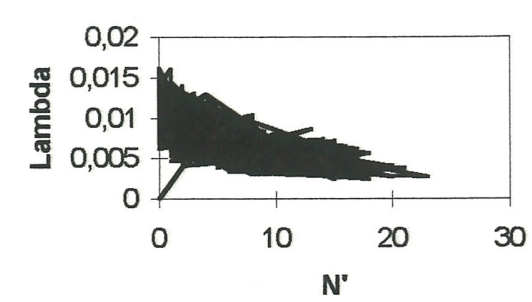

Figura 5.3: Trajetória das amostras não selecionadas de $\mathrm{N}^{\prime}$ e $\lambda$ $\mathrm{S}=\mathbf{2 5 0 0}$

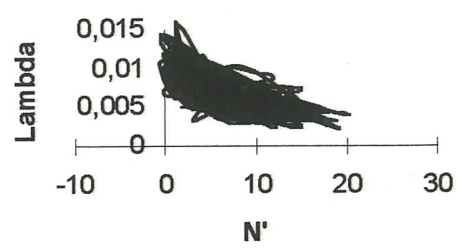

Figura 5.4: Trajetória das amostras selecionadas de $\mathrm{N}^{\prime}$ e $\lambda$ $\mathrm{S}=\mathbf{1 5 0 0}$

A figura 5.3 ilustra a trajetória das cadeias sem seleção e a figura 5.4 ilustra a trajetória das cadeias após seleção. É possível notar visualmente que a seleção dos dados permitiu a convergência das cadeias à uma distribuição de equilíbrio.

A seleção dos dados foi realizada de forma a garantir uma amostra não-correlacionada e independente. As funções de autocorrelação das amostras $S=1500$ podem ser ilustradas nas figuras (5.5) e (5.6).

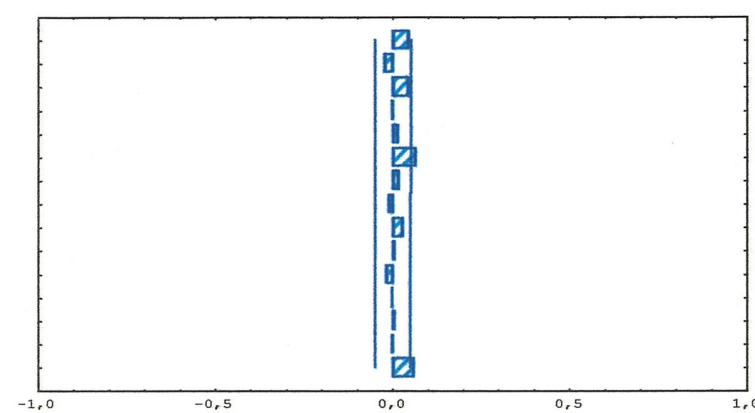

Figura 5.5: função de autocorrelação para $\lambda$

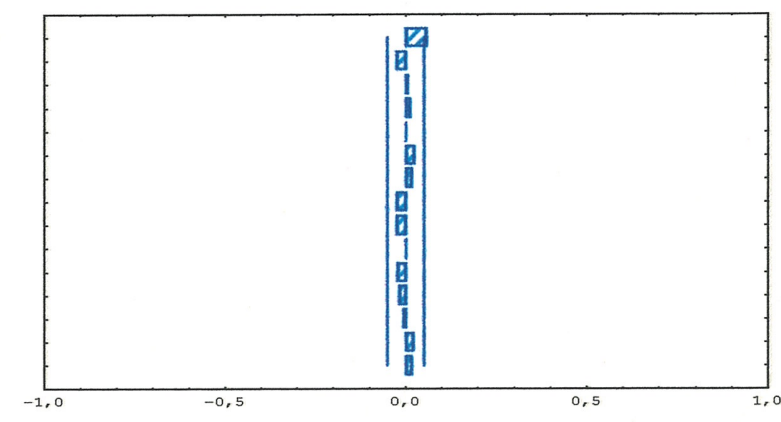

Figura 5.6: função de autocorrelação para $N^{\prime}$

Através das figuras (5.5) e (5.6), podemos perceber que as amostras simuladas pelo método Gibbs Sampling, são não correlacionadas, ou seja, as funções de autocorrelação ilustradas nas figuras acima, apresentam todos os termos próximos a zero.

Resultados: Considerando a amostra obtida $(\mathrm{S}=1500)$, através do algorítmo Gibbs Sampling, obtemos os resumos a posteriori descritos na tabela 5.2 e aproximações das densidades a posteriori marginais para $\lambda$ e $N^{\prime}$ são ilustradas nas figuras (5.7) e (5.8), respectivamente. 


\begin{tabular}{|l|l|l|l|l|}
\hline & Média & Mediana & SD & Int. Cred. 95\% \\
\hline$\lambda$ & 0.00676 & 6.0000 & 3.5561 & $(0.0035 ; 0.01103)$ \\
$\mathrm{N}^{\prime}$ & 6.1307 & 0.00654 & 0.00198 & $(1 ; 14)$ \\
\hline
\end{tabular}

Tabela 5.2: Resumos a posteriori para o modelo de JM considerando as densidades a priori (3.1)

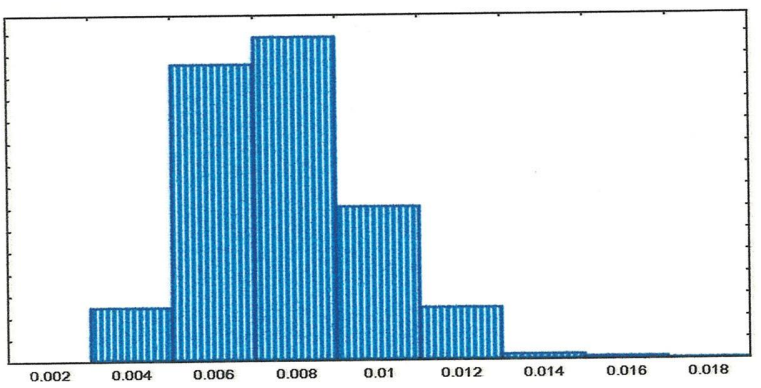

Figura 5.7: Aproximação da Densidade a Posteriori Marginal para $\mathbf{N}^{\prime}=\mathrm{N}-26$

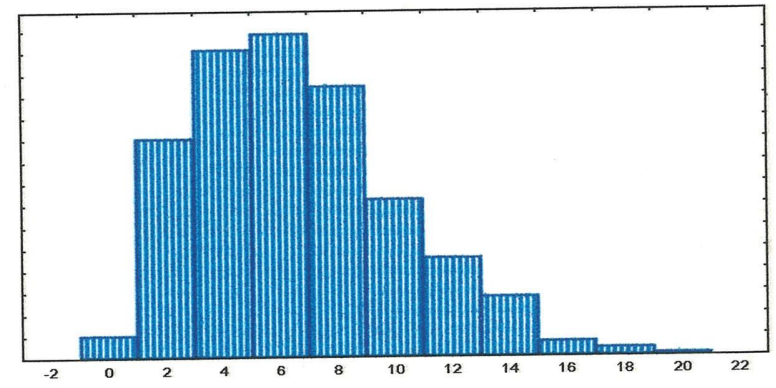

Figura 5.8: Aproximação da Densidade a Posteriori Marginal para $\lambda$

Comparacão entre as estimativas: Comparando os estimadores de máxima verossimilhança para $\lambda$ e $N\left(\hat{\lambda}=0.00685\right.$ e $\left.N^{\prime}=N-26=5.2159\right)$, obtidos através da Análise Clássica (ver seção 5.1), e as estimativas pontuais (Média e Mediana), obtidas através de uma Análise Bayesiana utilizando algorítmos de simulação (ver tabela 5.2), verificamos uma proximidade nos valores obtidos.

Já as estimativas por intervalo, obtidas através da Análise Bayesiana considerando as amostras geradas pelo algoritmo Gibbs Sampling (percentil 95\%), mostram-se mais precisas do que as estimativas intervalares (ver seção 5.1), obtidas segundo uma Análise Clássica via teoria assintótica.

Convergência: Um indicador de convergência é dado pelo fator de redução potencial estimada da escala ( R ), pela avaliação de sua proximidade a 1 (ver Gelman e Rubin (1992), apêndice(3)). Assim, considerando $\mathrm{S}=1500$ amostras geradas pelo algorímo Gibbs Sampling, para o modelo de Jelinski e Moranda, os fatores de redução de escala para $\lambda$ e N', são dados por,

$$
\begin{aligned}
\hat{\mathbf{R}}_{\lambda} & =1.00039, \\
\hat{\mathbf{R}}_{\mathrm{N}} & =1.00063 .
\end{aligned}
$$


Conclusão: Dessa forma, podemos admitir que a convergência foi atingida, ou seja, que as amostras, obtidas através do algorítmo Gibbs Sampling, convergem à distribuições de equilíbrio a qual estamos interessados.

Ainda dentro da verificação de convergência, é possível observarmos a convergência de forma visual, através das figuras (5.9) e (5.10), que mostram as trajetórias das cadeias ao longo das iterações.

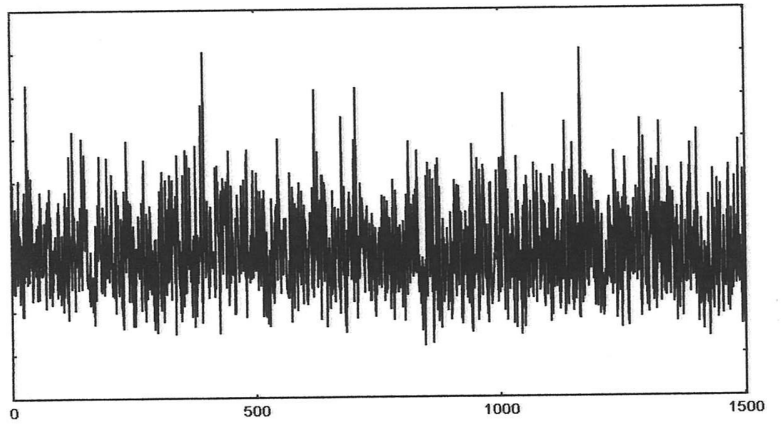

Figura 5.9: trajetórias das cadeias $-\lambda$

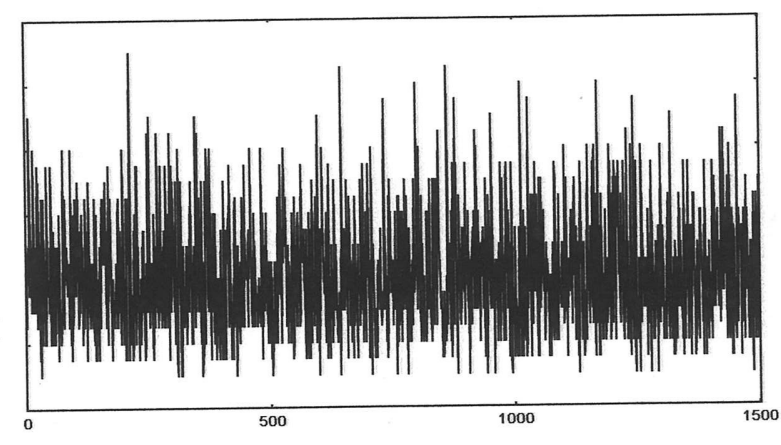

Figura 5.10: trajetórias das cadeias - $\mathrm{N}^{\prime}$

Através das figuras (5.9) e (5.10) podemos observar que após um período inicial, os gráficos apresentam repetidamente o mesmo comportamento qualitativo e quantitativo, podendo assim concluirmos pela convergência da amostra à distribuição de interesse.

\section{2 - Assumindo $\lambda$ com Priori Gama e N com Priori Binomial Negativa}

Assumindo o modelo de Jelinski e Moranda (2.1), consideramos as densidades a priori (3.9), com $\gamma=5, \delta=685, \mathrm{r}=15 \mathrm{e} \mathrm{p}=0.48$ baseados na informação a respeito dos dados.

Dessa forma, as densidades a priori para $\lambda$ e $N$, são dadas por,

$$
\lambda \sim \Gamma[5,685],
$$

e

$$
\mathrm{N} \sim \mathrm{BN}[15,0.48] .
$$


De (3.15), as distribuições condicionais de $\lambda / N^{\prime}, D$ e $N^{\prime} / \lambda, D$, requeridas para 0 algorítmo Gibbs Sampling, são dadas por,

$$
\lambda / N^{\prime}, D \sim \Gamma\left[31,685+250 N^{\prime}+\sum_{i=1}^{n} x_{i}\right]
$$

e

$$
\mathrm{N}^{\prime} / \lambda, \mathrm{D} \sim \mathrm{BN}\left[41,0.48 \mathrm{e}^{-250 \lambda}\right]
$$

onde D denota o conjunto de dados sobre tempos de falha $\left\{\mathrm{x}_{1}, \ldots, \mathrm{x}_{26}\right\}$ e $\mathrm{N}^{\prime}=\mathrm{N}-26$.

Selecão da amostra: Com as distribuições condicionais dadas acima, foram geradas cinco cadeias pelo algorítmo Gibbs Sampling, com 3000 iterações cada, considerando diferentes valores iniciais para cada cadeia. Para os parâmetros $\lambda$ e $\mathrm{N}^{\prime}$ foram selecionadas as $505^{\text {th }}, \ldots, 3000^{\text {th }}$ iterações, que de cinco cadeias produziu uma amostra de tamanho 2500.

Com essa forma de seleção, as amostras, investigadas através das suas respectivas funções de autocorrelação, mantiveram-se não-correlacionadas.

Resultados: Considerando $\mathrm{S}=2500$ amostras, geradas pelo algorítmo Gibbs Sampling, para $\lambda$ e $\mathrm{N}^{\prime}$, a tabela 5.3 mostra as estimativas pontuais e intervalares para os parâmetros em questão e as figuras (5.11) e (5.12) ilustram aproximações das densidades a posteriori marginais para $\lambda$ e $N^{\prime}$.

\begin{tabular}{|c|c|c|c|}
\hline & Média & Mediana & Int. Cred. 95\% \\
\hline$\lambda$ & 0.00772 & 0.007603 & $0.00463 ; 0.01145$ \\
$\mathrm{~N}^{\prime}$ & 3.728 & 3 & $0 ; 10$ \\
\hline
\end{tabular}

Tabela 5.3: Resumos a posteriori para o modelo de JM considerando as densidades a priori (3.9) 


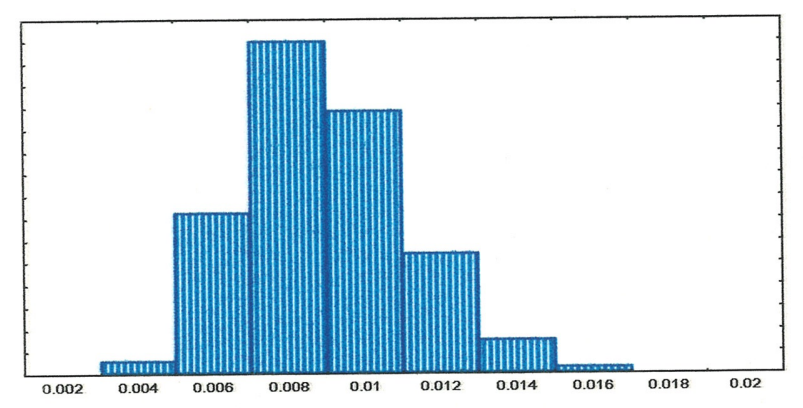

Figura 5.11: Aproximação da Densidade a Posteriori Marginal para $\lambda$

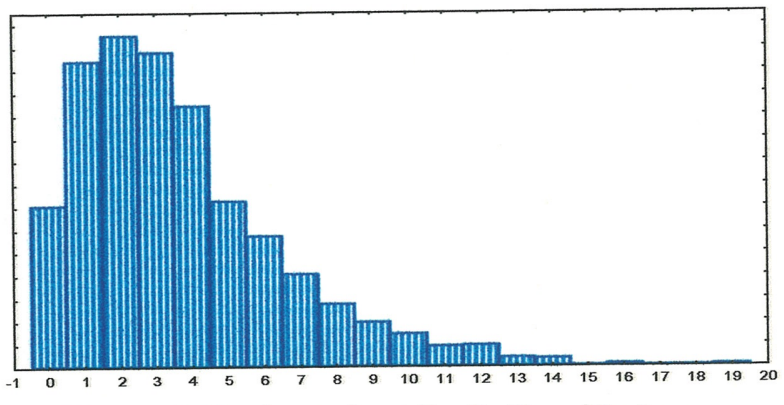

Figura 5.12: Aproximação da Densidade a Posteriori Marginal para $\mathbf{N}^{\prime}$

Comparacão entre as estimativas: Os resultados obtidos através de uma Análise Clássica onde os estimadores de máxima verossimilhança são dados por $\left(\hat{\lambda}=0.00685\right.$ e $\left.\mathrm{N}^{\prime}=\mathrm{N}-26=5.2159\right)$, mostram-se razoavelmente próximos aos resumos a posteriori pontuais (Média e Mediana), obtidos através da Análise Bayesiana, utilizando as amostras geradas pelo algorítmo Gibbs Sampling.

Já os intervalos de confiança 95\%, segundo a Análise Clássica, mostram-se menos precisos do que os intervalos de credibilidade obtidos via amostras geradas pelo algorítmo Gibbs Sampling.

Convergência: Considerando $\mathrm{S}=2500$ amostras, geradas pelo algorítmo Gibbs Sampling para o modelo de Jelinski e Moranda, os fatores de redução de escala para $\lambda$ e $\mathrm{N}^{\prime}$, são dados por,

$$
\begin{aligned}
& \hat{\mathbf{R}}_{\lambda}=1.003614, \\
& \hat{\mathbf{R}}_{\mathrm{N}^{\prime}}=1.002787 .
\end{aligned}
$$

Conclusão: Pela avaliação da proximidade dos fatores $\hat{\mathrm{R}}_{\lambda}$ e $\hat{\mathrm{R}}_{\mathrm{N}^{\prime}}$ a 1 , podemos concluir que as amostras geradas pelo algorítmo Gibbs Sampling convergem à distribuição de interesse.

\section{3 - Assumindo $\lambda$ com Priori Gama e N com Priori Logarítmica}


Assumindo ainda o modelo de Jelinski e Moranda, consideramos as densidades a priori (3.16) e (3.17) para $\lambda$ e N, onde (3.17) é denominada distribuição logarítmica (ver Yang, 1994).

Dessa forma, considerando $\gamma_{1}=5, \delta_{1}=685$ e $\theta=0.9$, baseados na informação contida nos dados, as densidades a priori para $\lambda$ e $\mathrm{N}^{\prime}$, são dadas por,

$$
\lambda \sim \Gamma[5,685]
$$

$\mathrm{e}$

$$
\mathrm{N} \sim \log \operatorname{aritmica}(0.9)
$$

Assim, de (3.24), as densidades a posteriori condicionais $\left(\lambda / N^{\prime}, D\right)$ e $\left(N^{\prime} / \lambda, D\right)$, requeridas para o algorítmo Gibbs Sampling, são dadas por,

$$
\lambda / \mathrm{N}^{\prime}, \mathrm{D} \sim \Gamma\left[31,685+250 \mathrm{~N}^{\prime}+\sum_{\mathrm{i}=1}^{\mathrm{n}} \mathrm{x}_{\mathrm{i}}\right]
$$

e

$$
\mathrm{N}^{\prime} / \lambda, \mathrm{D} \sim \mathrm{BN}\left[26,1-0.9 \mathrm{e}^{-250 \lambda}\right]
$$

onde D denota o conjunto dos tempos de falha $\left\{\mathrm{x}_{1}, \ldots, \mathrm{x}_{26}\right\}$ e $\mathrm{N}^{\prime}=\mathrm{N}-26$.

Selecão da amostra: Considerando as distribuições condicionais $\left(\lambda / N^{\prime}, D\right)$ e $\left(N^{\prime} / \lambda, D\right)$, foram geradas cinco cadeias pelo algorítmo Gibbs Sampling com 6000 iterações cada, assumindo diferentes valores iniciais para as cadeias.

Para os parâmetros $\lambda$ e $\mathrm{N}^{\prime}$ foram selecionamos as $1005^{\text {th }}, \ldots, 6000^{\text {th }}$ iterações, que de cinco cadeias produziu uma amostra de tamanho 5000 .

Com essa forma de seleção, as amostras investigadas através de suas respectivas funções de autocorrelação, mantiveram-se não correlacionadas.

Resultados: Os resultados podem ser visualizados na tabela (5.4) e nas figuras (5.13) e (5.14), onde temos os resumos a posteriori e aproximações para as densidades a posteriori marginais de $\lambda$ e $N^{\prime}$, respectivamente. 


\begin{tabular}{|c|c|c|c|}
\hline & Média & Mediana & Int. Cred. 95\% \\
\hline \hline$\lambda$ & 0.00707 & 0.00696 & $0.00376 ; 0.010998$ \\
$\mathrm{~N}^{\prime}$ & 5.629 & 5 & $0 ; 17$ \\
\hline
\end{tabular}

Tabela 5.4: Resumos a posteriori para o modelo de JM considerando as densidades a priori (3.16) e (3.17)

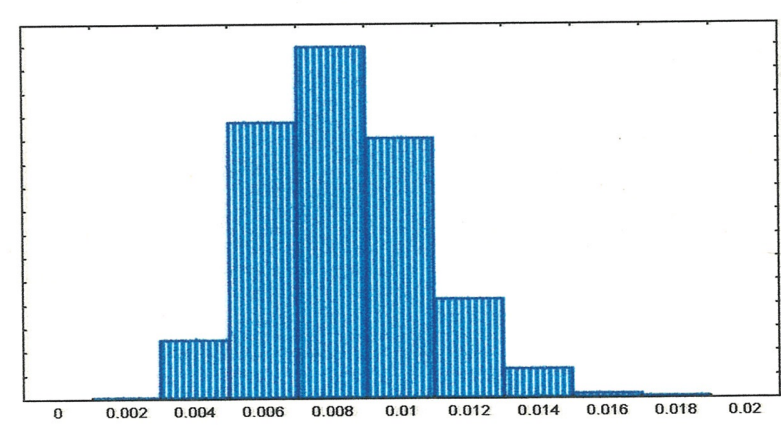

Figura 5.13: Aproximação da Densidade a Posteriori Marginal para $\lambda$

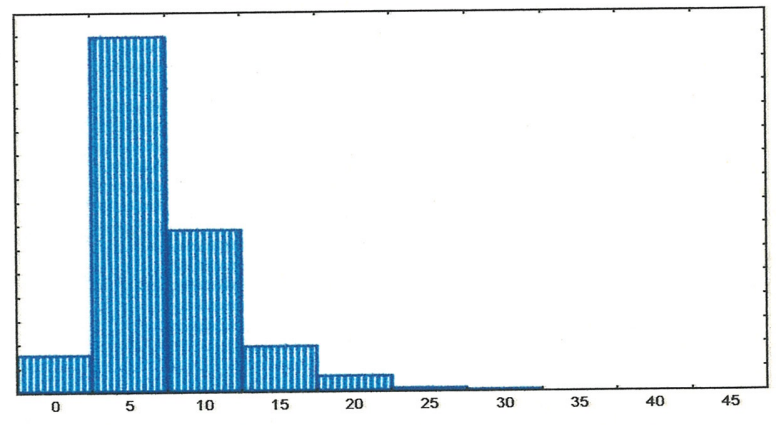

Figura 5.14: Aproximação da Densidade a Posteriori Marginal para $\mathbf{N}^{\prime}$

Comparacão entre as estimativas: Os resultados obtidos através das Análises Clássica e Bayesiana com o uso do algorítmo Gibbs Sampling, mostram-se muito próximos, embora haja uma maior precisão nas estimativas intervalares segundo uma Análise Bayesiana.

Convergência: Considerando $\mathrm{S}=5000$ amostras, geradas pelo algorítmo Gibbs Sampling, os fatores de redução de escala para $\lambda$ e $N^{\prime}$, são dados por,

$$
\begin{aligned}
\hat{\mathrm{R}}_{\lambda} & =1.000389, \\
\hat{\mathrm{R}}_{\mathrm{N}^{\prime}} & =1.004343 .
\end{aligned}
$$

Conclusão: Pela avaliação da proximidade dos fatores $\hat{\mathbf{R}}_{\lambda}$ e $\hat{\mathbf{R}}_{\mathrm{N}^{\prime}}$ a 1 , podemos concluir pela convergência das amostras à uma distribuição de equilíbrio. 
4 - Análise Bayesiana Hierárquica assumindo $N$ com Priori Poisson $(\theta), \theta$ com Priori Gama $(\alpha, \beta)$ e $\lambda$ com Priori Gama $\left(\gamma_{2}, \delta_{2}\right)$

Assumindo ainda o modelo de Jelinski e Moranda (2.1), consideramos nessa sub-seção o primeiro caso de densidades a priori hierárquicas, descritas em (3.25) e (3.26), onde os hiperparâmetros são dados por $\gamma_{2}=5, \delta_{2}=685, \theta=30, \alpha=60$ e $\beta=2$, baseados na informação a respeito dos dados.

Dessa forma, as densidades a priori para $\lambda, \mathrm{N}$ e $\theta$, são dadas por,

$$
\begin{aligned}
& \lambda \sim \Gamma[5,685], \\
& \mathrm{N} / \theta \sim \mathrm{P}(30),
\end{aligned}
$$

e

$$
\theta \sim \Gamma[60,2]
$$

De (3.30), as distribuições condicionais requeridas para o algorítmo Gibbs Sampling, são dadas por,

$$
\lambda / \mathrm{N}^{\prime}, \theta, \mathrm{D} \sim \Gamma\left[31,685+250 \mathrm{~N}^{\prime}+\sum_{\mathrm{i}=1}^{\mathrm{n}} \mathrm{x}_{\mathrm{i}}\right]
$$

$\mathrm{e}$

$$
\begin{gathered}
\mathrm{N}^{\prime} / \theta, \lambda, \mathrm{D} \sim \mathrm{P}\left(30 \mathrm{e}^{-250 \lambda}\right), \\
\theta \sim \Gamma\left[86+\mathrm{N}^{\prime}, 3\right],
\end{gathered}
$$

onde $\mathrm{N}^{\prime}=\mathrm{N}-26 \mathrm{e} \sum_{\mathrm{i}=1}^{\mathrm{n}} \mathrm{x}_{\mathrm{i}}=2492$.

Selecão da amostra: Com as distribuições condicionais dadas acima, foram geradas cinco cadeias pelo algorítmo Gibbs Sampling com 6000 iterações cada, considerando valores arbitrários para as cadeias. 
A seleção das observações geradas foi realizada de forma a obter amostras não correlacionadas, considerando as $1005^{\text {th }}, 1010^{\text {th }}, \ldots, 5000^{\text {th }}$ iterações, que de cinco cadeias produziu uma amostra de tamanho 5000.

Resultados: Considerando $\mathrm{S}=5000$ amostras geradas pelo algorítmo de Gibbs Sampling para $\lambda$, $\mathrm{N}^{\prime}$ e $\theta$, a tabela (5.5) esboça as estimativas pontuais e intervalares (de acordo com o percentil$95 \%$ ) para os parâmetros de interesse e as figuras (5.15),(5.16) e (5.17) ilustram aproximações das densidades a posteriori marginais para os parâmetros $\lambda, \mathrm{N}^{\prime}$ e $\theta$.

\begin{tabular}{|c|c|c|c|}
\hline & Média & Mediana & Int. Cred. 95\% \\
\hline \hline$\lambda$ & 0.00684 & 0.006656 & $0.00384 ; 0.01081$ \\
$N^{\prime}$ & 6.174 & 6 & $1 ; 15$ \\
$\theta$ & 30.76 & 30.67 & $24.62356 ; 38.1109$ \\
\hline
\end{tabular}

Tabela 5.5: Resumos a posteriori para o modelo de JM considerando as densidades a priori (3.25) e (3.26)

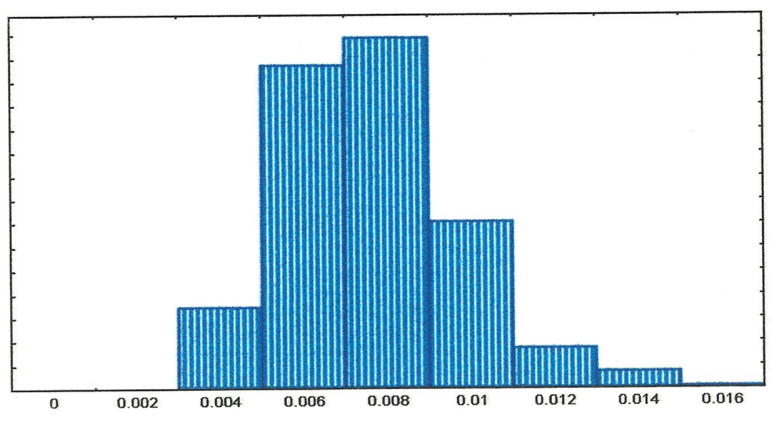

Figura 5.15: Aproximação da Densidade a Posteriori Marginal para $\lambda$

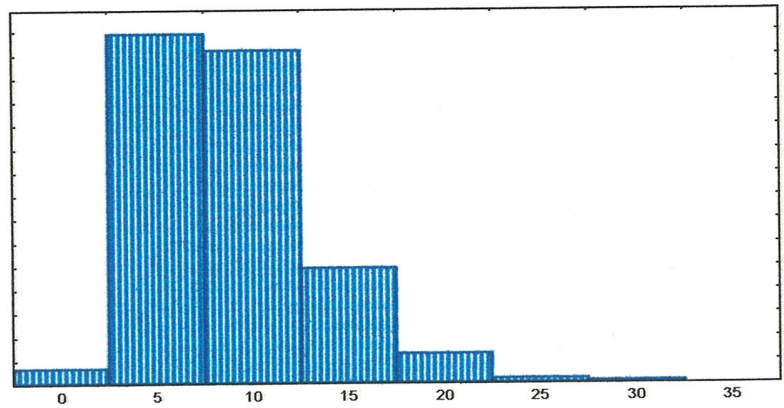

Figura 5.16: Aproximação da Densidade a Posteriori Marginal para $N^{\prime}$

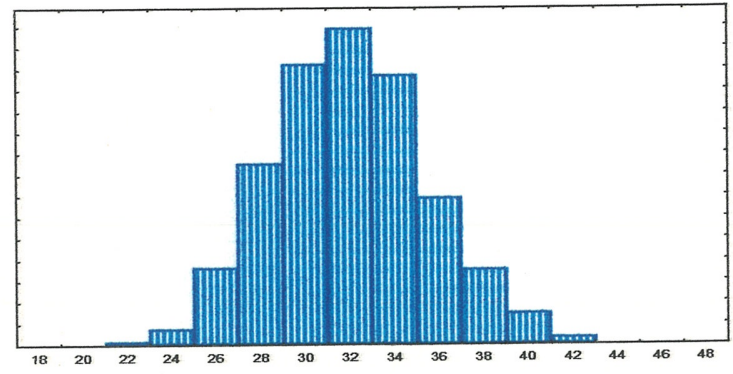

Figura 5.17: Aproximação da Densidade a Posteriori Marginal para $\theta$ 
Comparacão entre as estimativas: As estimativas pontuais obtidas através das duas Análises (Clássica e Bayesiana), mostram-se relativamente próximas. Já as estimativas intervalares, mostram-se mais precisas considerando a análise Bayesiana com o uso do algorítmo Gibbs Sampling.

Convergência: Considerando $S=5000$ amostras, os fatores de convergência de Gelman e Rubin, são dados por,

$$
\begin{aligned}
& \hat{\mathbf{R}}_{\lambda}=1.000888 \\
& \hat{\mathbf{R}}_{\mathrm{N}^{\prime}}=1.001789 \\
& \hat{\mathbf{R}}_{\theta}=1.002132 .
\end{aligned}
$$

Conclusão: Podemos concluir pela convergência das amostras à uma distribuição de equilíbrio devido a proximidade dos fatores de redução de escala $\hat{\mathbf{R}}_{\lambda}, \hat{\mathbf{R}}_{N}$ e $\hat{\mathbf{R}}_{\theta}$ a 1 .

\section{5 - Análise Bayesiana Hierárquica assumindo $\mathbf{N}$ com Priori Poisson $(\theta), \theta$ com Priori Gama $(\alpha, \beta), \lambda$ com Priori Gama $\left(\gamma_{2}, \delta_{2}\right)$ e $\delta_{2}$ com Priori Gama $(\xi, \eta)$.}

Assumindo ainda o modelo de Jelinski e Moranda (2.1), consideramos o segundo caso de densidades a priori hierárquicas, descritas em (3.35) e (3.31), onde os hiperparâmetros dessa densidades a priori, obtidos segundo uma análise preliminar baseada na informação contida nos dados, são dados por $\theta=30, \alpha=60, \beta=2, \gamma_{2}=5, \delta_{2}=685$ e $\xi=685 \eta=1$.

Portanto, as densidades a priori para $\lambda, \mathrm{N}, \theta$ e $\delta_{2}$, são dadas por,

$$
\begin{gathered}
\mathrm{N} / \theta \sim \mathrm{P}(30), \\
\theta \sim \Gamma[60,2], \\
\lambda \sim \Gamma[5,685], \\
\delta_{2} \sim \Gamma[685,1] .
\end{gathered}
$$


De (3.34), as densidades condicionais requeridas para o algorítmo Gibbs Sampling, são dadas por,

$$
\begin{gathered}
\lambda / \mathrm{N}^{\prime}, \theta, \delta_{2}, \mathrm{D} \sim \Gamma\left[31,685+250 \mathrm{~N}^{\prime}+\sum_{\mathrm{i}=1}^{\mathrm{n}} \mathrm{x}_{\mathrm{i}}\right], \\
\delta_{2} / \lambda, \mathrm{N}^{\prime}, \theta, \mathrm{D} \sim \Gamma(690,1+\lambda), \\
\mathrm{N}^{\prime} / \theta, \lambda, \delta_{2}, \mathrm{D} \sim \mathrm{P}\left(30 \mathrm{e}^{-250 \lambda}\right), \\
\theta / \mathrm{N}^{\prime}, \lambda, \delta_{2}, \mathrm{D} \sim \Gamma\left[86+\mathrm{N}^{\prime}, 3\right] .
\end{gathered}
$$

onde $\mathrm{N}^{\prime}=\mathrm{N}-26, \sum_{\mathrm{i}=1}^{\mathrm{n}} \mathrm{x}_{\mathrm{i}}=2492$ e $\mathrm{D}$ denota o conjunto dos tempos de falha $\left\{\mathrm{x}_{1}, \ldots, \mathrm{x}_{\mathrm{n}}\right\}$

Selecão da amostra: Com as distribuições condicionais dadas acima, foram geradas cinco cadeias pelo algorítmo Gibbs Sampling, com 6000 iterações cada, considerando diferentes valores iniciais para cada cadeia. Essas amostras, para cada parâmetro, foram selecionadas considerando as $1005^{\text {th }}, \ldots, 6000^{\text {th }}$, que de cinco cadeias produziu uma amostra de tamanho 5000 .

Resultados: A tabela 5.6 esboça os resumos a posteriori para os parâmetros $\mathrm{N}^{\prime}, \lambda, \theta_{2}, \delta_{2}$ e as figuras (5.18), (5.19), (5.20) e (5.21) ilustram aproximações das densidades a posteriori marginais de interesse para esses parâmetros.

\begin{tabular}{|c|c|c|c|}
\hline & Média & Mediana & Int. Cred. 95\% \\
\hline$\lambda$ & 0.006823 & 0.00665 & $0.00375 ; 0.01081$ \\
$\mathrm{~N}^{\prime}$ & 6.205 & 6 & $1 ; 16$ \\
$\theta$ & 30.79 & 30.68 & $24.43019 ; 37.57693$ \\
$\delta_{2}$ & 685.9 & 685.8 & $634.229 ; 730.6751$ \\
\hline
\end{tabular}

Tabela 5.5: Resumos a posteriori para o modelo de JM considerando as densidades a priori (3.25) e (3.31) 


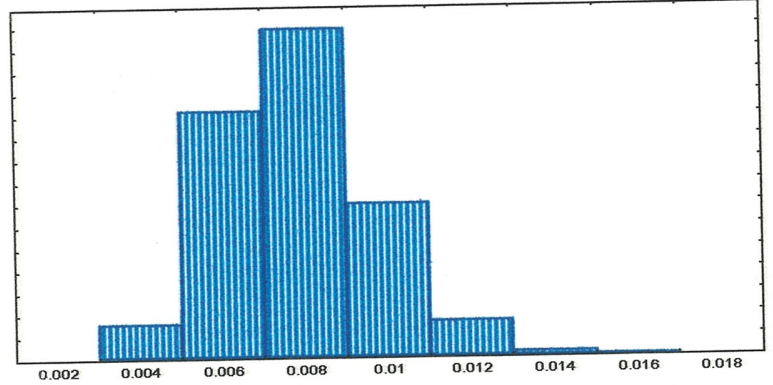

Figura 5.18: Aproximação da Densidade a Posteriori Marginal para $\lambda$

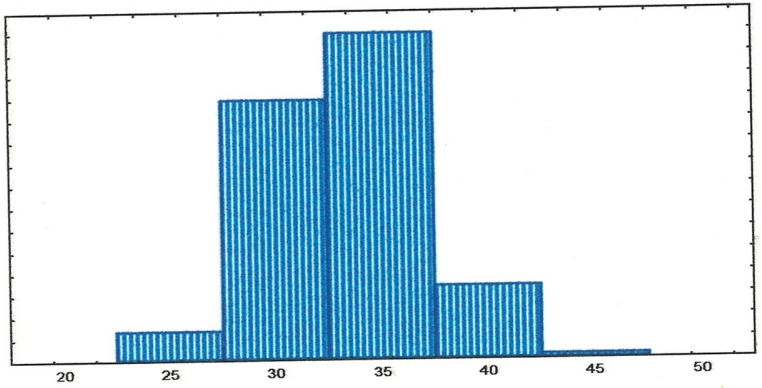

Figura 5.20: Aproximação da Densidade a Posteriori Marginal para $\theta$

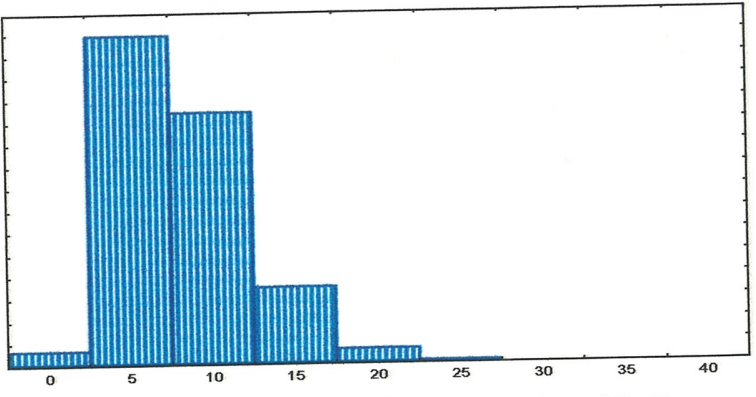

Figura 5.19: Aproximação da Densidade a Posteriori Marginal para $\mathbf{N}^{\prime}$

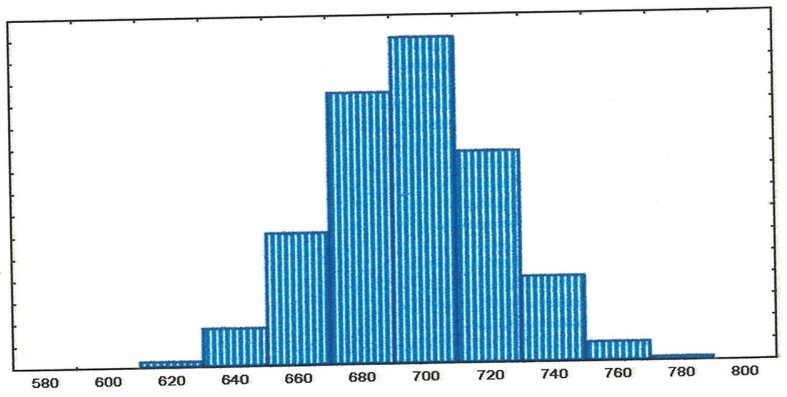

Figura 5.21: Aproximação da Densidade a Posteriori Marginal para $\delta_{2}$

Comparacão entre as estimativas: As estimativas pontuais obtidas pela análise Clássica e Bayesiana para $\mathrm{N}^{\prime}=\mathrm{N}-26$ ( o número de erros no software) e para a taxa de falha $(\lambda)$, mostram-se relativamente próximas.

Já as estimativas intervalares, mostram-se mais precisas considerando uma análise Bayesiana utilizando algorítmos de simulação.

Convergência: Considerando $\mathrm{S}=5000$ amostras, obtidas pelo algorítmo Gibbs Sampling, os fatores de redução de escala para $\lambda, N^{\prime}, \theta$ e $\delta_{2}$, para avaliar a convergência, são dados por, 


$$
\begin{aligned}
& \hat{\mathbf{R}}_{\lambda}=1.001531, \\
& \hat{\mathbf{R}}_{\mathrm{N}^{\prime}}=1.004429, \\
& \hat{\mathbf{R}}_{\theta}=1.002508, \\
& \hat{\mathbf{R}}_{\delta_{2}}=1.000883 .
\end{aligned}
$$

Conclusão: Avaliando a proximidade dos fatores de redução de escala para $\lambda, \mathrm{N}, \theta$ e $\delta_{2}$ a 1 , podemos admitir que a convergência foi atingida, ou seja, que as amostras convergem a uma distribuição de equilíbrio.

Comparacão entre as distribuicões a Priori adotadas: Considerando diferentes densidades a priori para os parâmetros $\mathrm{N}$ (o número de erros no software) e $\lambda$ (a taxa de falha do software), englobando também densidades a priori hierárquicas, no modelo de Jelinski e Moranda, podemos notar uma proximidade de valores razoável nas estimativas pontuais e intervalares.

Outro ponto interessante, é que todos os casos mostram uma maior precisão nas estimativas intervalares, comparadas à uma análise Clássica. 


\subsubsection{Análise Bayesiana para os Dados NTDS sob o Modelo de Moranda.}

Considerando os dados da tabela (5.1) e o modelo de Moranda (2.22), assumimos as

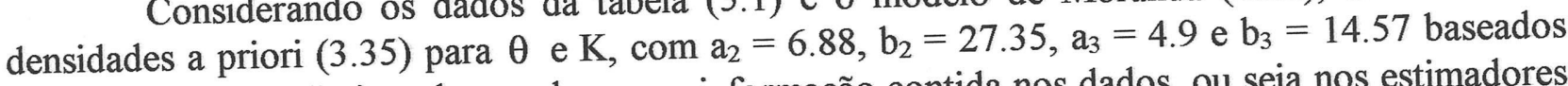
em uma análise preliminar de acordo com a informação contida nos dados, ou seja nos estimadores de máxima verossimilhança. Portanto,

$$
\theta \sim \Gamma[6.88,27.35]
$$

e

$$
\mathrm{K} \sim \mathrm{B}[4.9,14.57]
$$

De (3.8), as distribuições condicionais de $(\theta / K, D)$ e $(K / \theta, D)$ são dadas por,

$$
\theta / \mathrm{K}, \mathrm{D} \sim \Gamma\left[32.88,27.35+\sum_{\mathrm{i}=1}^{\mathrm{n}} \mathrm{K}^{\mathrm{i}-1} \mathrm{t}_{\mathrm{i}}\right]
$$

e

$$
\pi(\mathrm{K} / \theta, \mathrm{D}) \propto \mathrm{K}^{4.9-1}(1-\mathrm{K})^{14.57-1} \psi_{1}(\theta, \mathrm{K})
$$

onde $\psi_{1}(\theta, K)=\exp \left\{325 \ln \mathrm{K}-\theta \sum_{\mathrm{i}=1}^{\mathrm{n}} \mathrm{t}_{\mathrm{i}}\right\}$ e $\mathrm{D}$ denota o conjunto dos tempos entre falhas $\left\{\mathrm{t}_{1}\right.$, $\left.\ldots, \mathrm{t}_{\mathrm{n}}\right\}$.

Selecão da Amostra: Com as distribuições condicionais dadas acima, foram geradas cinco cadeias com 1200 iterações cada, considerando segundo uma análise preliminar dos dados e os valores dos estimadores de máxima verossimilhança para $\theta$ e $\mathrm{K}$, diferentes valores iniciais para as cadeias.

Para cada parâmetro foram selecionados as $205^{\text {th }}, 210^{\text {th }}, \ldots, 1195^{\text {th }}, 1200^{\text {th }}$ iterações, que de cinco cadeias produziu uma amostra de tamanho 1000. 
Essa seleção foi realizada de forma a garantir uma baixa correlação entre a amostra. Esse fato pode ser confirmado através das figuras (5.22) e (5.23) que ilustram as funções de autocorrelação das amostras geradas.

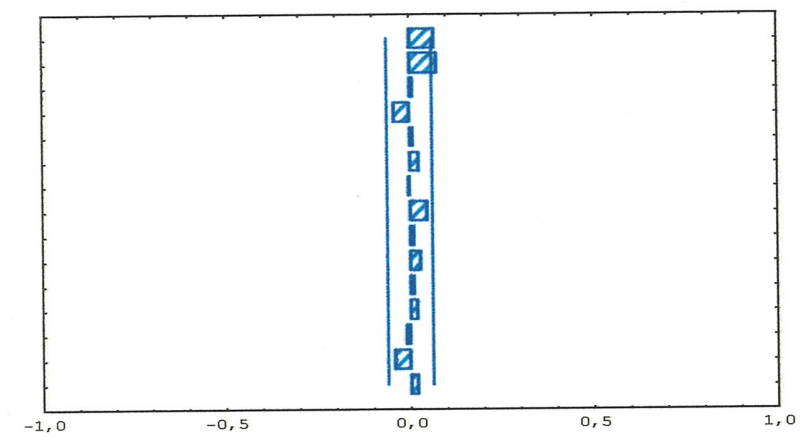

Figura 5.22: função de autocorrelação para $\theta$

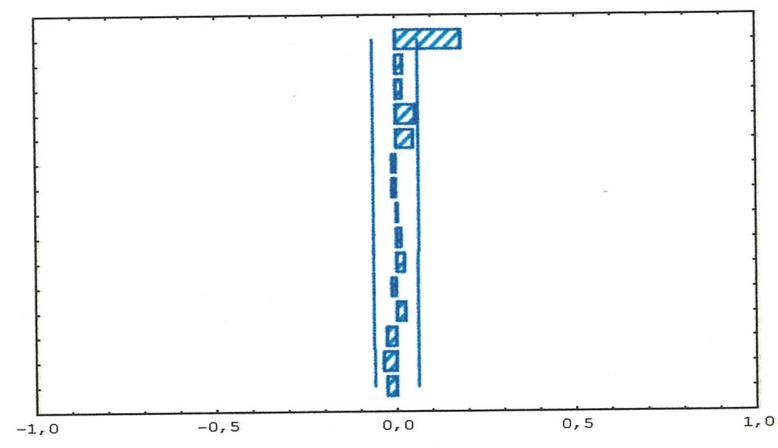

Figura 5.23: função de autocorrelação para $K$

Conclusão: Através das figuras 5.22 e 5.23, percebemos que todos os termos são próximos a zero, indicando que as amostras são não correlacionadas.

Resultados: Considerando as amostras não-correlacionadas, geradas pelo algorítmo Gibbs Sampling com Metrópolis Hastings, a tabela (5.7) esboça os resumos a posteriori para os parâmetros $\theta$ e K e as figuras (5.24) e (5.25) ilustram aproximações das densidades a posteriori marginais para $\theta$ e $\mathrm{K}$.

\begin{tabular}{|c|c|c|c|}
\hline & Média & Mediana & Int. Cred. 95\% \\
\hline \hline$\theta$ & 0.22289 & 0.22044 & $(0.159996 ; 0.301432)$ \\
$\mathrm{K}$ & 0.94512 & 0.94592 & $(0.919098 ; 0.968689)$ \\
\hline
\end{tabular}

Tabela 5.7: Resumos a posteriori para o modelo de Moranda 


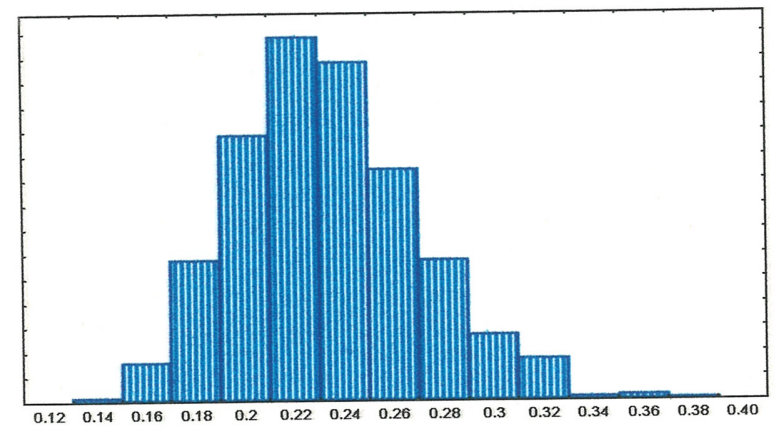

Figura 5.24: Aproximação da densidade a Posteriori Marginal de $\theta$

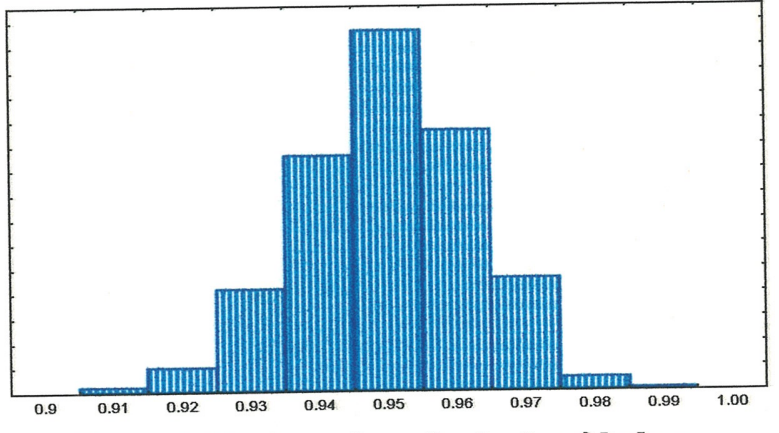

Figura 5.25: Aproximação da densidade a Posteriori marginal de $\mathbf{K}$

Comparacão entre as estimativas: Podemos perceber que os estimadores de máxima verossimilhança para $\theta$ e $\mathrm{K}$, obtidos através da Análise Clássica $(\hat{\theta}=0.251633$ e $\hat{\mathrm{K}}=0.936947$ ), são muito próximos às estimativas pontuais, obtidas através das amostras geradas pelo algorítmo Gibbs Sampling.

Já os intervalos de confiança para $\theta$ e K (95\%), obtidos segundo a teoria assintótica, mostram-se menos precisos do que os intervalos de credibilidade $(95 \%)$ via amostras Gibbs (ver tabela 5.7).

Convergência: Considerando o fator de convergência $\hat{R}$, descrito por Gelman e Rubin (1992) e assumindo $\mathrm{S}=1000$ amostras geradas pelo algorítmo Gibbs Sampling para o modelo de Moranda, os fatores de redução de escala $(\hat{\mathbf{R}})$ para $\theta$ e K podem ser dados por,

$$
\begin{aligned}
& \hat{\mathrm{R}}_{\theta}=1.00004, \\
& \hat{\mathrm{R}}_{\mathrm{K}}=1.00122 .
\end{aligned}
$$

Conclusão: Pela avaliação da proximidade a 1 , dos fatores $\hat{\mathbf{R}}_{\theta}$ e $\hat{\mathbf{R}}_{\mathrm{K}}$, podemos admitir que a convergência ocorreu e que essa amostra converge à distribuição de interesse. 


\subsubsection{Análise Bayesiana para os Dados NTDS sob o Modelo de Goel e Okumoto.}

Considerando o modelo de Goel e Okumoto (2.41) e os dados NTDS (tabela 1) assumimos as densidades a priori (3.41) para $\lambda, \mathrm{N} \mathrm{e} \mathrm{p}$, onde $\mathrm{a}_{4}=5, \mathrm{~b}_{4}=685, \theta_{2}=30, \mathrm{a}_{5}=2.5$ e $\mathrm{b}_{5}$ $=2.5$ são constantes conhecidas obtidas através de uma análise preliminar dos dados.

Dessa forma as densidades a priori para $\lambda, \mathrm{N}$ e $\mathrm{p}$, são dadas por,

$$
\begin{gathered}
\lambda \sim \Gamma[5,685], \\
\mathrm{N} \sim \mathrm{P}[30], \\
\mathrm{p} \sim \mathrm{B}[2.5,2.5] .
\end{gathered}
$$

De (3.44), as densidades condicionais de $(\lambda / \mathrm{N}, \mathrm{p}, \mathrm{D}),(\mathrm{N} / \mathrm{p}, \lambda, \mathrm{D}) \mathrm{e}(\mathrm{p} / \mathrm{N}, \lambda, \mathrm{D})$, são dadas por,

$$
\begin{gathered}
\lambda / \mathrm{N}, \mathrm{p}, \mathrm{D} \sim \Gamma\left[31,685+\mathrm{p} \sum_{\mathrm{i}=1}^{26} \mathrm{x}_{\mathrm{i}}+(\mathrm{N}-26 \mathrm{p}) \mathrm{x}_{\mathrm{n}}\right], \\
\pi(\mathrm{N} / \lambda, \mathrm{p}, \mathrm{D}) \propto \frac{\mathrm{e}^{-30} 30^{\mathrm{N}}}{\mathrm{N} !} \psi_{2}(\mathrm{~N}, \mathrm{p}, \lambda),
\end{gathered}
$$

onde $\psi_{2}(\mathrm{~N}, \mathrm{p}, \lambda)=\exp \{\ln \mathrm{A}(\mathrm{N}, \mathrm{p})-250(\mathrm{~N}-26 \mathrm{p}) \lambda\} \mathrm{e}$

$$
\pi(\mathrm{p} / \mathrm{N}, \lambda, \mathrm{D}) \propto \mathrm{p}^{2.5-1}(1-\mathrm{p})^{2.5-1} \psi_{3}(\mathrm{~N}, \mathrm{p}, \lambda)
$$

onde $\psi_{3}(\mathrm{~N}, \mathrm{p}, \lambda)=\exp \left\{\ln \mathrm{A}(\mathrm{N}, \mathrm{p})-\mathrm{p} \lambda \sum_{\mathrm{i}=1}^{\mathrm{n}} \mathrm{x}_{\mathrm{i}}-250(\mathrm{~N}-26 \mathrm{p}) \lambda\right\}$ 
Selecão da Amostra: Considerando as densidades condicionais acima, foram geradas cinco cadeias pelo algorítmo Gibbs Sampling com Metrópolis Hastings com 2.000 iterações cada, considerando diferentes valores iniciais para as cadeias, baseados numa análise preliminar dos dados.

Para os parâmetros $\lambda, \mathrm{N}$ e p selecionamos as $405^{\text {th }}, 410^{\text {th }}, \ldots, 1199^{\text {th }}, 2000^{\text {th }}$, que de cinco cadeias produziu uma amostra de tamanho 2000.

A seleção dessa amostra foi feita de forma a garantir independência e uma pequena autocorrelação dos valores obtidos, que pode ser visualizada através das figuras (5.26), (5.27) e (5.28).

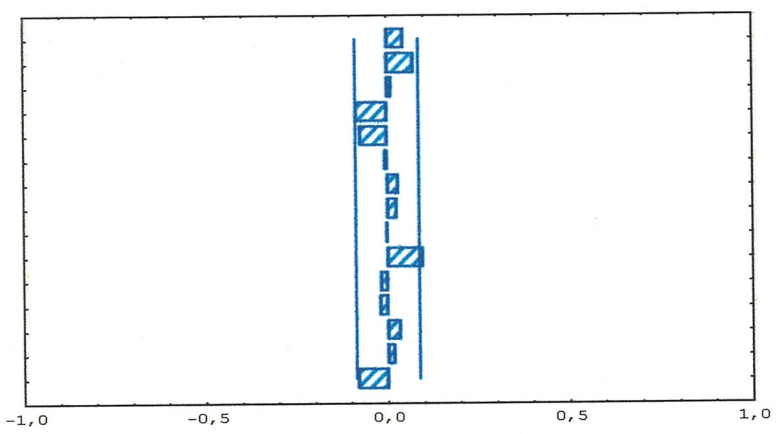

Figura 5.26: função de autocorrelação para $\lambda$

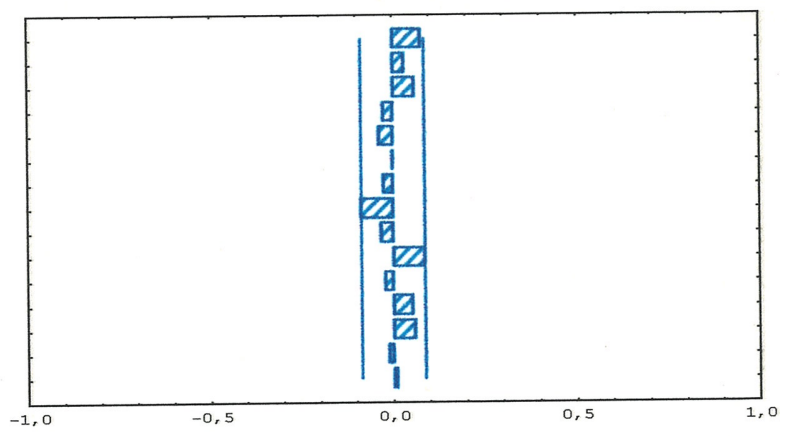

Figura 5.27: função de autocorrelação para $\mathbf{N}$

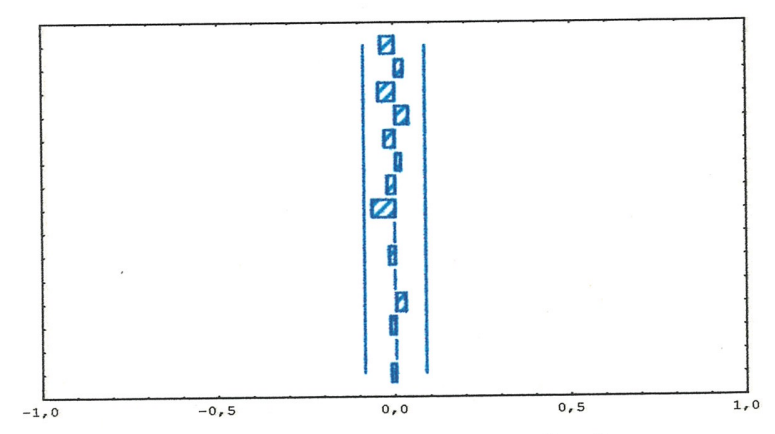

Figura 5.28: função de autocorrelação para p

Conclusão: Podemos observar através das figuras 5.265 .27 e 5.28 que as amostras não são correlacionadas, devido a maioria dos termos apresentarem-se próximos a zero. 
Resultados: Considerando as $\mathrm{S}=2000$ amostras, obtidas através do método Gibbs Sampling com Metrópolis Hastings, a tabela (5.8) mostra os resumos a posteriori para os parâmetros $\lambda, \mathrm{N}$ e p e as figuras (5.29), (5.30) e (5.31) mostram aproximações das densidades a posteriori marginais para $\lambda, \mathrm{N}$ e $\mathrm{p}$.

\begin{tabular}{|c|c|c|c|c|}
\hline & Média & Mediana & SD & Int. Cred. 95\% \\
\hline \hline$\lambda$ & 0.00634 & 0.00582 & 0.00257 & $0.002980 ; 0.0126824$ \\
$\mathrm{~N}$ & 28.272 & 28. & 5.091 & $19 ; 39$ \\
$\mathrm{p}$ & 0.62189 & 0.64403 & 0.17523 & $0.241834 ; 0.905575$ \\
\hline
\end{tabular}

Tabela 5.8: Resumos a posteriori para o modelo de Goel e Okumoto

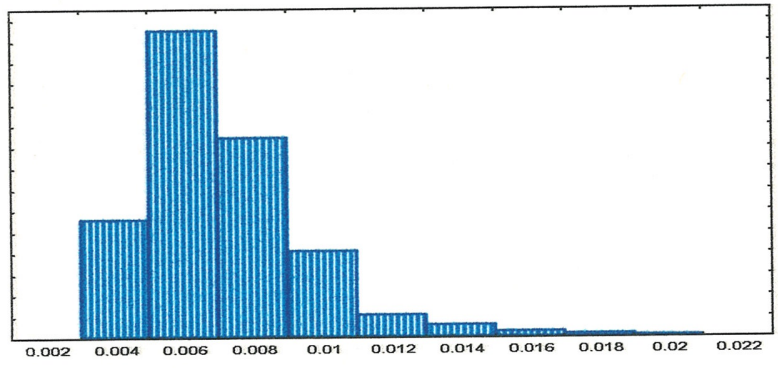

Figura 5.29: Aproximação da densidade a posteriori marginal para $\lambda$

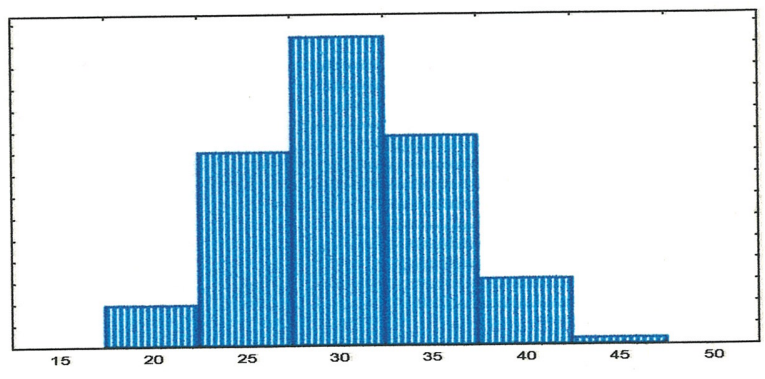

Figura 5.30: Aproximação da densidade a posteriori marginal para $N$

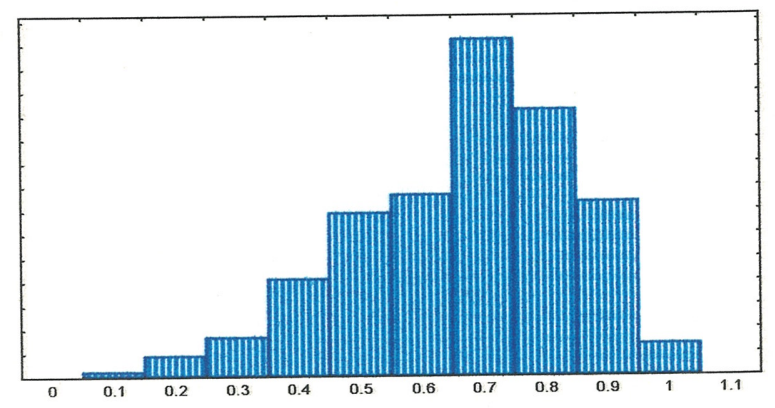

Figura 5.30: Aproximação da densidade a posteriori marginal para $\mathbf{p}$ 
Convergência: Assumindo as amostras $\mathrm{S}=2000$ para o modelo de Goel e Okumoto, consideramos o fator de redução de escala $(\hat{R})$ para $\lambda, N$ e p, dados por,

$$
\begin{aligned}
& \hat{\mathrm{R}}_{\lambda}=1.00213, \\
& \hat{\mathrm{R}}_{\mathrm{N}}=1.00716, \\
& \hat{\mathrm{R}}_{\mathrm{p}}=1.00148 .
\end{aligned}
$$

Conclusão: Avaliando a proximidade dos fatores $\hat{\mathrm{R}}_{\lambda}, \hat{\mathrm{R}}_{\mathrm{N}}$ e $\hat{\mathrm{R}}_{\mathrm{p}}$ a 1 , podemos admitir a convergência da amostra à distribuição de interesse.

\subsubsection{Análise Bayesiana para os Dados NTDS sob o Modelo de Schick e Wolverton.}

Considerando os dados introduzidos por Jelinski e Moranda (tabela 5.1) e o modelo de Schick e Wolverton descrito na seção (3.7), assumimos as densidades a priori (3.45) para $\lambda$ e $N$, com $\mathrm{a}_{6}=6.25, \mathrm{~b}_{6}=625$ e $\theta_{3}=30$, obtidos através de uma análise preliminar dos dados. Portanto, as densidades a priori para $\lambda$ e $N$, são dadas por,

$$
\begin{gathered}
\lambda \sim \Gamma[6.25,625], \\
\mathrm{N} \sim \mathrm{P}[30] .
\end{gathered}
$$

Dessa forma, de (3.49), as densidades a posteriori condicionais requeridas para o algorítmo Gibbs Sampling são dadas por,

$$
\lambda / \mathrm{N}^{\prime}, \mathrm{D} \sim \Gamma\left[32.25,625+\frac{\mathrm{N}}{2} \sum_{\mathrm{i}=1}^{26} \mathrm{t}_{\mathrm{i}}^{2}-\frac{1}{2} \sum_{\mathrm{i}=1}^{26}(\mathrm{i}-1) \mathrm{t}_{\mathrm{i}}^{2}\right],
$$


e

$$
\mathrm{N}^{\prime} / \lambda, \mathrm{D} \sim \mathrm{P}\left[30 \mathrm{e}^{-\lambda \sum_{\mathrm{i}=1}^{26} \mathrm{t}_{\mathrm{i}}^{2} / 2}\right]
$$

onde $\mathrm{N}^{\prime}=\mathrm{N}-26$ e D denota o conjunto de tempos entre falhas $\left\{\mathrm{t}_{1}, \ldots, \mathrm{t}_{\mathrm{n}}\right\}$.

Selecão da Amostra: Com as distribuições condicionais dadas acima, foram geradas cinco cadeias com 2000 iterações cada, assumindo valores iniciais arbitrários para as cadeias, baseados numa análise preliminar utilizando informação dos dados.

Para os parâmetros foram selecionadas as $203^{\text {th }}, 206^{\text {th }}, \ldots, 2.000^{\text {th }}$, que de cinco cadeias produziu uma amostra de tamanho 3000 .

A seleção dessa amostra foi realizada de forma a garantir independência e uma baixa correlação entre os dados. Numa forma visual isso pode ser verificado através dos gráficos das funções de autocorrelação dados pelas figuras (5.32) e (5.33).

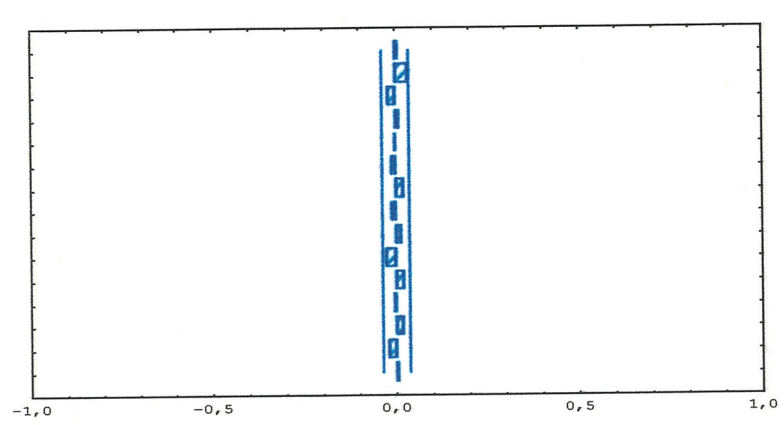

Figura 5.32: função de autocorrelação para $\operatorname{amostra} \mathbf{N}^{\prime}$

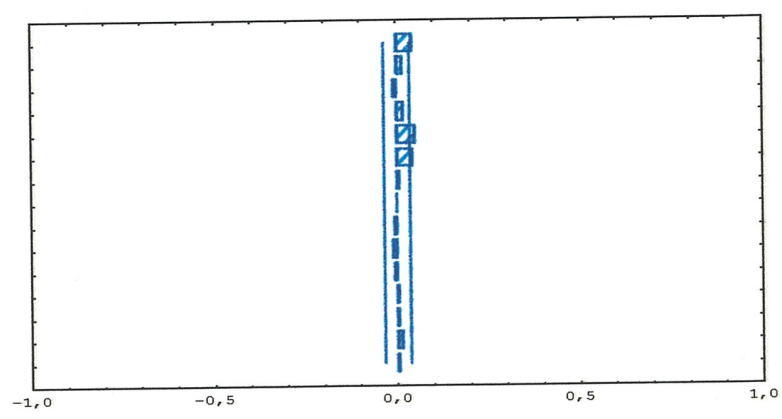

Figura 5.33: função de autocorrelação para amostra $\lambda$

Resultados: Considerando as $\mathrm{S}=3000$ amostras, obtidas pelo algorítmo Gibbs Sampling, a tabela (5.9) mostra os resumos a posteriori para os parâmetros $\lambda$ e $N^{\prime}$ e as figuras (5.34) e (5.35) mostram aproximações das densidades a posteriori marginais para os mesmos. 


\begin{tabular}{|c|c|c|c|c|}
\hline & Média & Mediana & SD & Int. Cred. 95\% \\
\hline$\lambda$ & 0.00133 & 0.00131 & 0.000243 & $0.0008919,0.001842$ \\
$\mathrm{~N}^{\prime}$ & 0.056667 & 0.06 & 0.253266 & 0,1 \\
\hline
\end{tabular}

Tabela 5.9: Resumos a posteriori para o modelo de SW

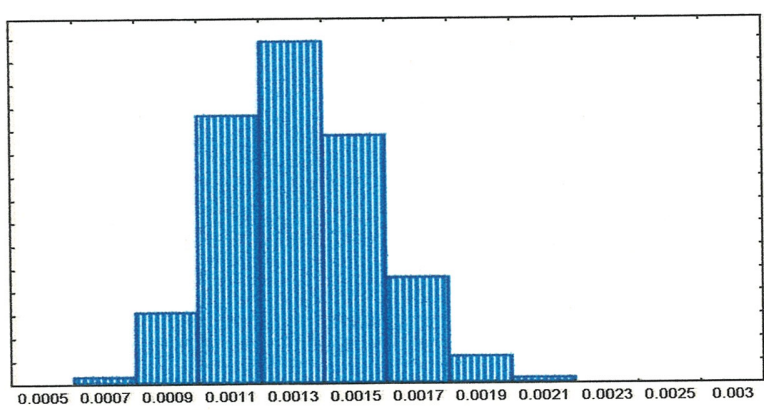

Figura 5.34: Aproximação da densidade a posteriori marginal de $\lambda$

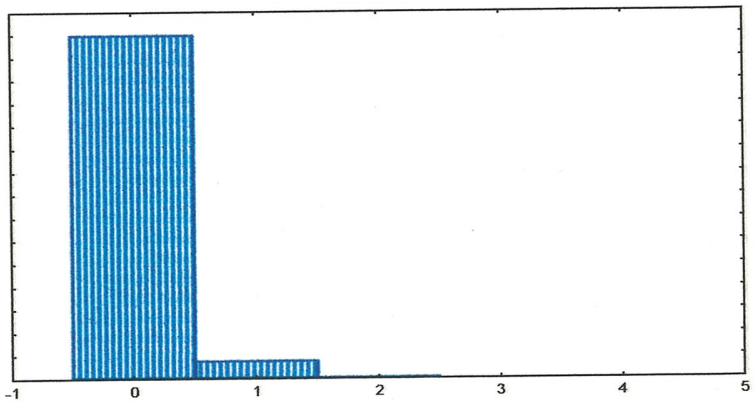

Figura 5.35: Aproximação da densidade a posteriori marginal de $\mathbf{N}^{*}$

Comparacão entre as estimativas: Podemos perceber que as estimativas pontuais e intervalares para $\lambda$ e $\mathrm{N}$, obtidas através das Análises Clássica e Bayesiana (amostras geradas pelo algorítmo Gibbs Sampling), não mostram-se muito próximas.

Convergência: Os fatores de redução de escala para $\lambda$ e N' podem ser dados por,

$$
\begin{aligned}
& \hat{\mathrm{R}}_{\lambda}=1.00001, \\
& \hat{\mathrm{R}}_{\mathrm{N}}{ }^{\prime}=1.0016 .
\end{aligned}
$$

Conclusão: Avaliando a proximidade dos fatores $\hat{\mathrm{R}}_{\lambda}$ e $\hat{\mathrm{R}}_{\mathrm{N}}{ }^{\prime}$ a 1 , podemos concluir pela convergência das amostras à uma distribuição de equilíbrio. 


\subsubsection{Análise Bayesiana para os Dados NTDS sob o Modelo de Littlewood e Verral.}

Considerando os dados da tabela (5.1) e o modelo Bayesiano de Littlewood e Verral descrito na seção 3.8, assumimos as densidades a priori (3.52) para $\alpha, \beta_{0}$ e $\beta_{1}$, com $a_{7}=1, b_{7}=$ $0.3, a_{8}=10, b_{8}=100, a_{9}=20$ e $b_{9}=1$, obtidos através de uma análise preliminar dos dados. Portanto,

$$
\begin{gathered}
\alpha \sim \Gamma[1,0.3], \\
\beta_{1} \sim \Gamma[10,100],
\end{gathered}
$$

e

$$
\pi\left(\beta_{0} / \beta_{1}\right) \propto \alpha\left(\beta_{0}+\beta_{1}\right)^{20-1} \exp \left\{-\left(\beta_{0}+\beta_{1}\right)\right\}
$$

De (3.55), as densidades a posteriori condicionais requeridas para o algorítmo Gibbs Sampling com Metrópolis Hastings, são dadas por,

$$
\begin{gathered}
\alpha / \beta_{0}, \beta_{1}, \mathrm{D} \sim \Gamma\left[27, \gamma_{1}+0.3\right], \\
\pi\left(\beta_{0} / \beta_{1}, \mathrm{D}\right) \propto\left(\beta_{0}+\beta_{1}\right)^{20-1} \mathrm{e}^{-\left(\beta_{0}+\beta_{1}\right)} \psi_{4}\left(\alpha, \beta_{0}, \beta_{1}\right),
\end{gathered}
$$

onde $\psi_{4}\left(\alpha, \beta_{0}, \beta_{1}\right)=\exp \left\{-\gamma_{1} \alpha-\gamma_{2}\right\}$

$$
\pi\left(\beta_{1} / \alpha, \beta_{0}, D\right) \propto \beta_{1}^{10-1} e^{-100 \beta_{1}} \psi_{5}\left(\alpha, \beta_{0}, \beta_{1}\right)
$$

onde $\psi_{5}\left(\alpha, \beta_{0}, \beta_{1}\right)=\exp \left\{(20-1) \ln \left(\beta_{0}+\beta_{1}\right)-\beta_{1}-\gamma_{1} \alpha-\gamma_{2}\right\}$. 
Selecão da Amostra: Com as distribuições condicionais dadas acima, geramos dez cadeias pelo algorítmo Gibbs Sampling com Metrópolis Hastings, com 1.000 iterações cada, onde foram assumidos diferentes valores iniciais para as cadeias baseados numa análise preliminar dos dados.

Para cada parâmetro selecionamos as $208^{\text {th }}, 216^{\text {th }}, \ldots, 1000^{\text {th }}$ iterações, que de dez cadeias produziu uma amostra de tamanho 1.000 .

A seleção dessa amostra foi feita de forma a garantir independência e uma baixa correlação entre os dados obtidos, que pode ser visualizada através das figuras $(5.36),(5.37)$ e (5.38).

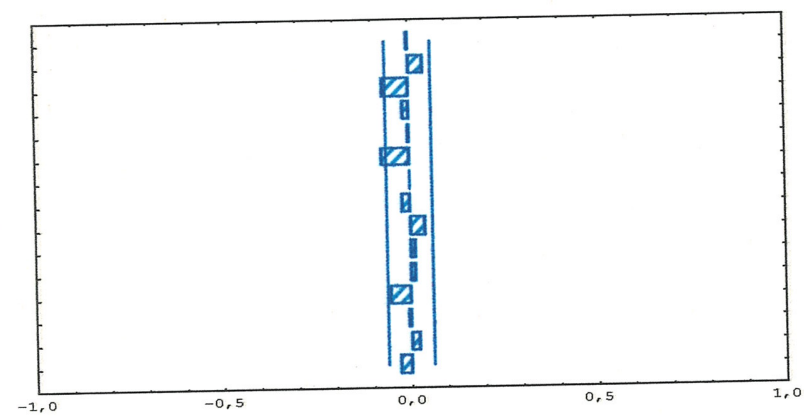

Figura 5.36: função de autocorrelação para amostra $\beta_{1}$

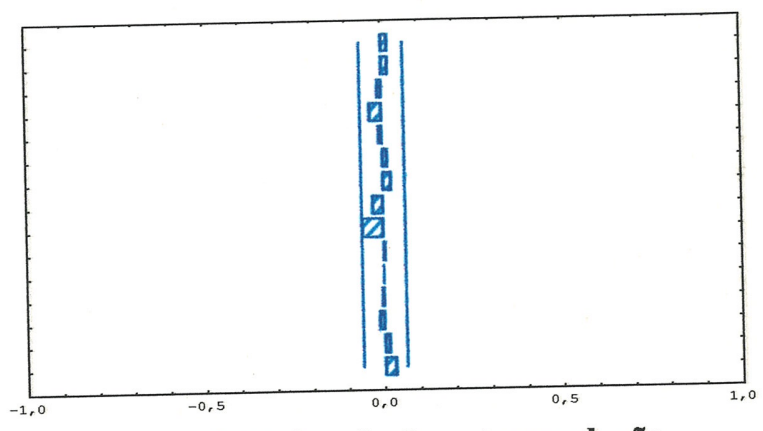

Figura 5.37: função de autocorrelação para amostra $\alpha$

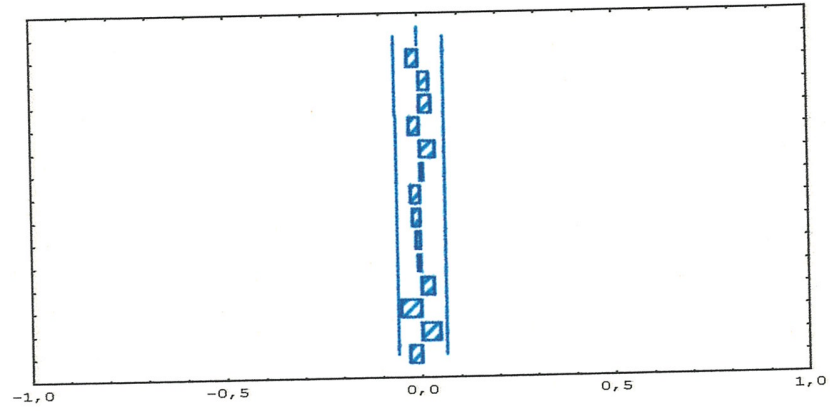

Figura 5.38: função de autocorrelação para amostra $\beta_{0}$

Conclusão: As figuras (5.36), (5.37) e (5.38) mostram os gráficos das funções de autocorrelação para as amostras obtidas pelo algorítmo Gibbs Sampling com Metrópolis Hastings, para os 
parâmetros $\alpha, \beta_{0}$ e $\beta_{1}$. Podemos observar através dos gráficos, todos os termos próximos a zero, o que nos leva a concluir que as amostras são não correlacionadas.

Resultados: Considerando as amostras não correlacionadas $(S=1.000)$, obtidas pelo método Gibbs Sampling com Metrópolis Hastings, mostramos na tabela (5.10) estimativas pontuais e intervalares para os parâmetros $\alpha, \beta_{0}$ e $\beta_{1}$ e nas figuras (5.39) (5.40)e (5.41) aproximações das densidades a posteriori marginais para $\alpha, \beta_{0}$ e $\beta_{1}$.

\begin{tabular}{|c|c|c|c|c|}
\hline & Média & Mediana & SD & Int. Cred. 95\% \\
\hline \hline$\alpha$ & 3.3529 & 3.3028 & 0.8078 & $(2.00847 ; 5.07887)$ \\
$\beta_{0}$ & 19.944 & 19.603 & 4.390 & $(12.2048 ; 29.0531)$ \\
$\beta_{1}$ & 0.09931 & 0.09598 & 0.03166 & $(0.047212 ; 0.174667)$ \\
\hline
\end{tabular}

Tabela 5.10: Resumos a posteriori para o modelo de Littlewood e Verral

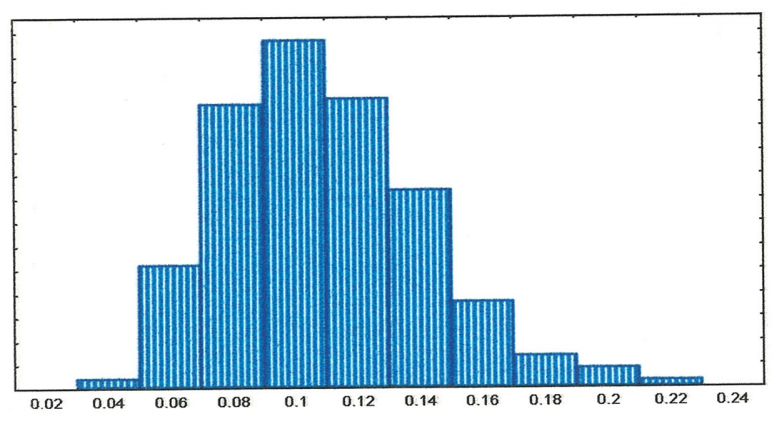

Figura 5.39: Aproximação da densidade a Posteriori marginal de $\beta_{1}$

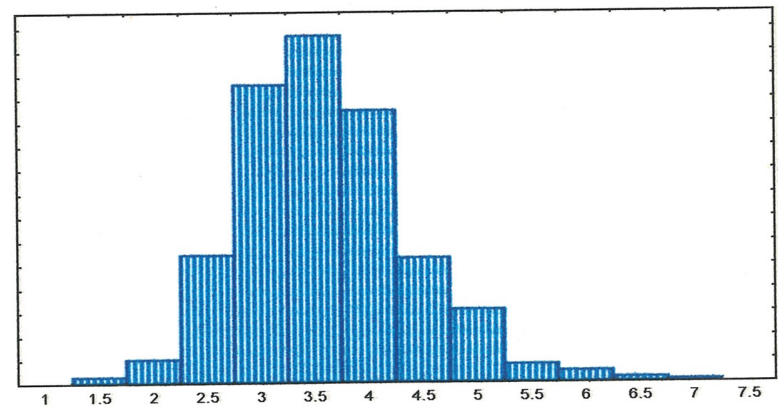

Figura 5.40: Aproximação da densidade a Posteriori marginal de $\alpha$

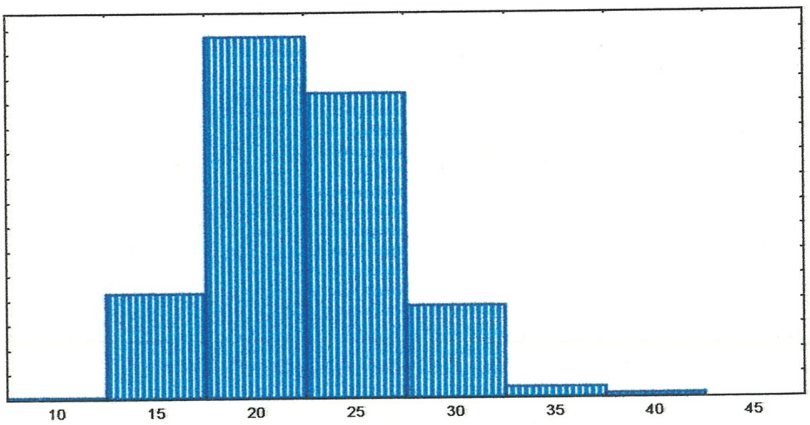

Figura 5.41: Aproximação da densidade a Posteriori marginal de $\beta_{0}$ 
Convergência: A convergência das amostras geradas pelo algorímto Gibbs Sampling com Metrópolis Hastings $(\mathrm{S}=1.000)$ pode ser monitorada pelo algorítmo de Gelman e Rubin (1992) onde os fatores de redução de escala para $\alpha, \beta_{0}$ e $\beta_{1}$ podem ser dados por,

$$
\begin{aligned}
& \hat{\mathbf{R}}_{\alpha}=1.00159, \\
& \hat{\mathbf{R}}_{\beta_{0}}=0.99924, \\
& \hat{\mathbf{R}}_{\beta_{1}}=1.00174 .
\end{aligned}
$$

Conclusão: Avaliando a proximidade dos fatores $\hat{\mathrm{R}}_{\alpha}, \hat{\mathrm{R}}_{\beta_{0}}$ e $\hat{\mathrm{R}}_{\beta_{1}}$ a 1 , podemos admitir que a amostra converge à distribuição de equilíbrio.

Em uma forma visual, consideramos os gráficos que mostram as trajetórias das cadeias ao longo das iterações através das figuras (5.42), (5.43) e (5.44).

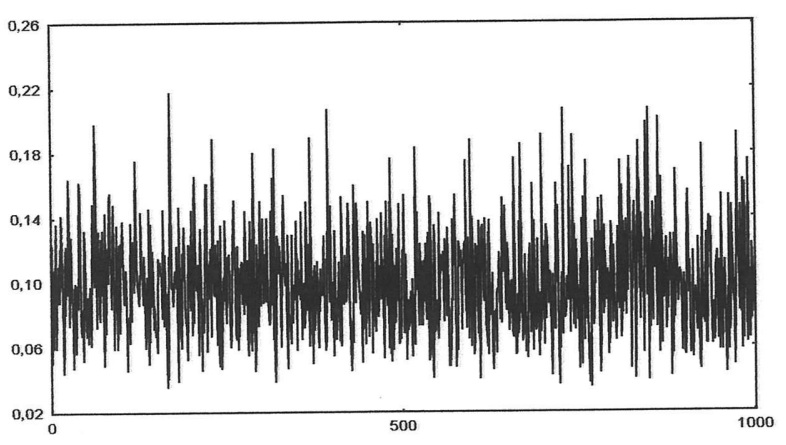

Figura 5.42: Trajetória das cadeias $-\beta_{1}$

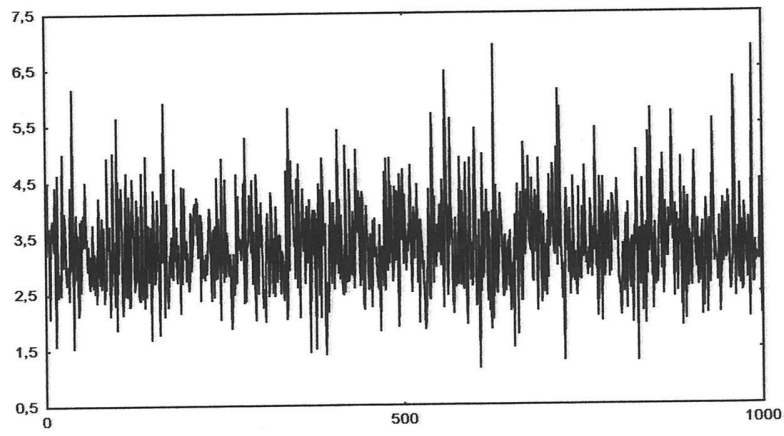

Figura 5.43: Trajetória das cadeias - $\alpha$

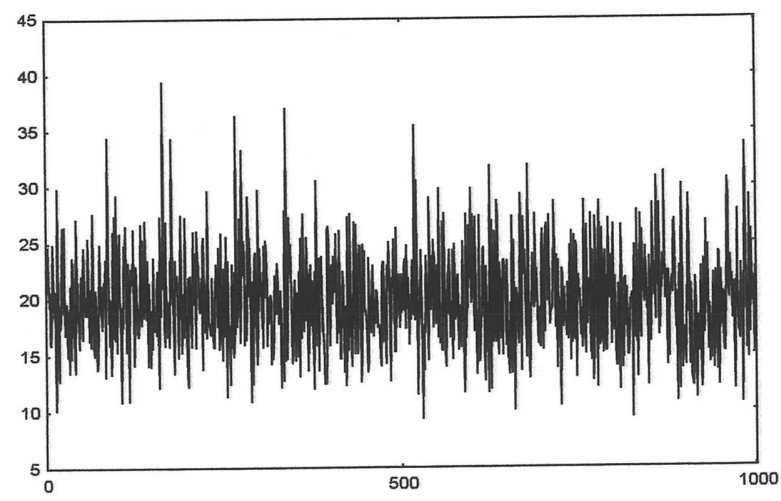

Figura 5.44: Trajetória das cadeias $-\beta_{0}$ 
Conclusão: Através dessas figuras, observamos que ocorreu a convergência, dado o comportamento repetitivo das cadeias após um período inicial.

\subsection{Seleção de Modelos}

O procedimento gráfico, descrito no capítulo 4, pode ser adotado para verificarmos o melhor modelo dentre os modelos estudados.

Considerando os dados da tabela 5.1, apresentamos na tabela (5.11) os valores das densidades preditivas $c_{i}=f\left(t_{i} \mid D_{(i)}\right)$ avaliados nos valores observados $t_{i}$ e aproximados por um estimador de Monte Carlo baseado nas amostras geradas pelo algorítmo Gibbs Sampling, para cada modelo considerado.

$\mathrm{Na}$ figura (5.45), apresentamos os gráficos de $\mathrm{c}_{\mathrm{i}}$ contra i para todos os cinco modelos em questão e na tabela (5.11), temos também os valores de $c(1)=\prod_{i=1}^{26} c_{i}$, considerando os diferentes modelos. 


\begin{tabular}{|c|c|c|c|c|c|}
\hline $\mathrm{i}$ & $\mathrm{LV}$ & $\mathrm{JM}$ & $\mathrm{SW}$ & $\mathrm{GO}$ & $\mathrm{MO}$ \\
\hline 1 & 0,03247 & 0,030965 & 0,0755903 & 0,036218 & 0,029982 \\
2 & 0,021305 & 0,017602 & 0,0367796 & 0,022907 & 0,017105 \\
3 & 0,024475 & 0,02223 & 0,0506388 & 0,027207 & 0,022431 \\
4 & 0,074242 & 0,087329 & 0,0953618 & 0,081413 & 0,0877 \\
5 & 0,044452 & 0,049473 & 0,0986254 & 0,050981 & 0,050768 \\
6 & 0,108453 & 0,123912 & 0,0529042 & 0,108578 & 0,119125 \\
7 & 0,061968 & 0,071294 & 0,0947465 & 0,068415 & 0,071063 \\
8 & 0,038143 & 0,043113 & 0,0887319 & 0,044542 & 0,044787 \\
9 & 0,061887 & 0,070389 & 0,0882486 & 0,067466 & 0,069084 \\
10 & 0,044582 & 0,051357 & 0,0899458 & 0,051205 & 0,051902 \\
11 & 0,130682 & 0,12515 & 0,0211354 & 0,112529 & 0,111052 \\
12 & 0,052321 & 0,059181 & 0,0830276 & 0,057838 & 0,057717 \\
13 & 0,129671 & 0,114975 & 0,0185271 & 0,106627 & 0,100666 \\
14 & 0,033162 & 0,039802 & 0,07625 & 0,040008 & 0,040296 \\
15 & 0,07314 & 0,07262 & 0,056227 & 0,070967 & 0,066853 \\
16 & 0,128187 & 0,099096 & 0,0146012 & 0,097535 & 0,086744 \\
17 & 0,087125 & 0,07553 & 0,0377261 & 0,076013 & 0,068474 \\
18 & 0,08694 & 0,072056 & 0,0341827 & 0,074076 & 0,065745 \\
19 & 0,052265 & 0,05179 & 0,0527312 & 0,053429 & 0,049112 \\
20 & 0,126264 & 0,076726 & 0,0093412 & 0,084955 & 0,070993 \\
21 & 0,025527 & 0,03229 & 0,0538004 & 0,032668 & 0,031895 \\
22 & 0,00299 & 0,007039 & 0,0062214 & 0,005317 & 0,007178 \\
23 & 0,044801 & 0,039756 & 0,0329447 & 0,045058 & 0,040493 \\
24 & 0,000174 & 0,000529 & 0,0000004 & 0,000167 & 0,000376 \\
25 & 0,10258 & 0,044299 & 0,0054276 & 0,063214 & 0,051825 \\
26 & 0,123496 & 0,04047 & 0,0013967 & 0,065062 & 0,052464 \\
\hline $\mathrm{C}(\mathrm{l})$ & $4.1 \times 10^{-36}$ & $3.2 \times 10^{-36}$ & $2.0 \times 10^{-43}$ & $2.8 \times 10^{-36}$ & $1.6 \times 10^{-36}$ \\
& & & & & \\
\hline
\end{tabular}

Tabela 5.11 - Valores de $c_{i}=f\left(t_{i} / D_{(i)}\right), i=1,2, \ldots ., 26$ 


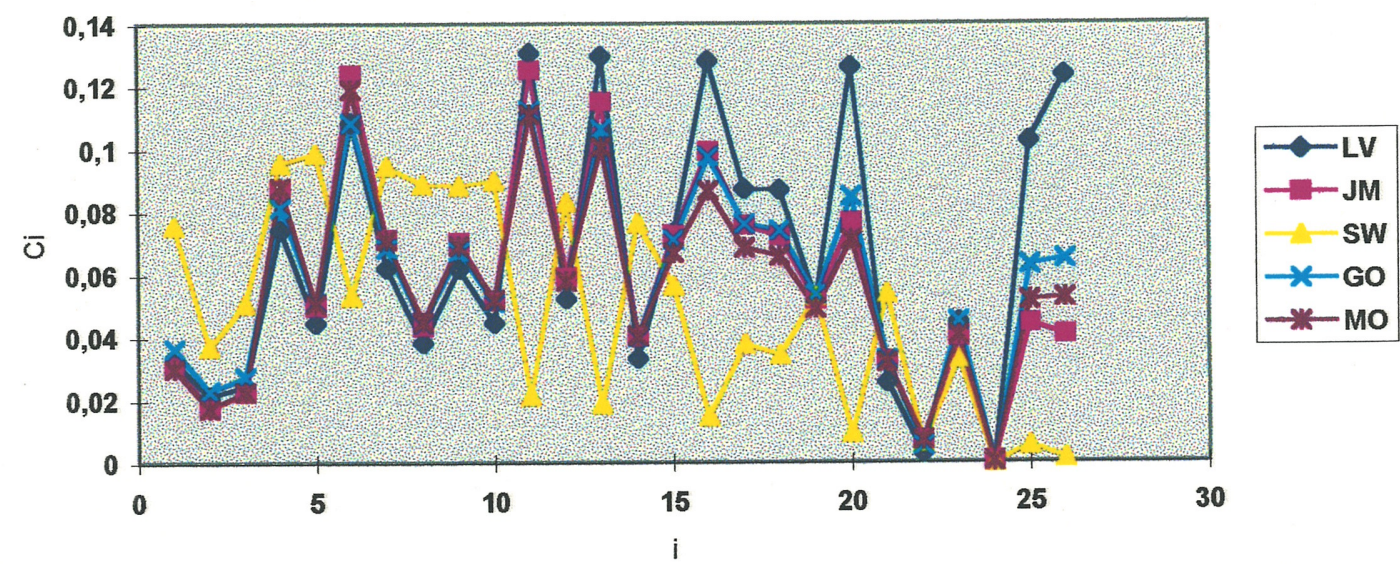

Figura (5.45): Plot de Ci versus i para os modelos LV, JM, SW, GO e Mo

Conclusão: Da tabela (5.11) e figura (5.45), observamos que o melhor modelo que maximiza c(1) $=\prod_{i=1}^{26} c_{i}$ é o modelo de Littlewood e Verral.

Ainda observando a tabela (5.11) e figura (5.45) é possível verificar que o modelo de Littlewood e Verral apresenta uma grande parte dos CPO'S acima dos CPO'S dos modelos alternativos, principalmente se observarmos os últimos valores verificamos que o modelo LV revela melhores predições para as últimas observações.

Portanto, o melhor modelo de confiabilidade de software (considerando tempos entre falhas do software) avaliado pela técnica CPO é o modelo Bayesiano de Littlewood e Verral. 
Capítulo 6

\section{CONCLUSÕES FINAIS E PERSPECTIVAS FUTURAS}

O uso de métodos de simulação via cadeias de Markov com Monte Carlo (MCMC) pode ser uma alternativa prática de grande interesse nas aplicações de confiabilidade de software usando modelos de estratégia tipo I, pois em geral nessas áreas existem informações prévias de especialistas que podem ser modeladas por uma densidade a priori e o enfoque Bayesiano é a forma apropriada para análise dos dados.

Os algorítmos relacionados ao procedimento MCMC (algorítmos Gibbs Sampling e Metrópolis Hastings) são de fácil implementação e não exigem muito esforço computacional para obtenção das quantidades a posteriori de interesse.

As alternativas computacionais aos algorítmos MCMC (método de aproximação de Laplace, métodos numéricos ou métodos de importance sampling) podem ser invariáveis, dada a existência de muitos parâmetros nos modelos formulados.

Além disso, estimadores de Monte Carlo para densidades preditivas são de simples obtenção e permitem uma comparação e discriminação entre os modelos considerados.

As técnicas desenvolvidas neste trabalho também poderiam ser extendidas a outros modelos na modelagem dos tempos entre falhas e também para modelos de estratégia tipo II, que consideram o número de erros encontrados num dado intervalo de tempo (modelos envolvendo processos de Poisson homogêneos e não-homogêneos). 
Apêndice 1

\section{O ALGORÍTMO GIBBS SAMPLING}

O algorítmo Gibbs Sampling é um esquema iterativo de amostragem de uma cadeia de Markov cujo núcleo de transição é formado pelas distribuições condicionais.

Para descrever o algorítmo, suponha que a distribuição de interesse é $\pi(\theta)$ onde $\theta=\left\{\theta_{1}, \ldots, \theta_{p}\right\}$ e que as densidades condicionais a posteriori $\pi_{i}\left(\theta_{i}\right)=\pi\left(\theta_{i} / \theta_{-i}\right) i=1, \ldots, p$ estão disponíveis.

Esse algorítmo fornece uma alternativa de geração de uma amostra de $\pi(\theta)$ (se está supondo que a geração direta de $\pi(\theta)$ é extremamente complicada e/ou custosa) baseada em sucessivas gerações das distribuições condicionais $\pi_{\mathrm{i}}\left(\theta_{\mathrm{i}}\right)$. Ele é descrito da seguinte forma:

(i) Inicialize o contador de iterações da cadeia $\mathrm{j}=1$ e arbitre valores iniciais $\theta^{(0)}=\left(\theta_{1}^{(0)}, \ldots, \theta_{\mathrm{p}}^{(0)}\right)$.

(ii) Obtenha um novo valor $\theta^{(\mathrm{j})}=\left(\theta_{1}^{(\mathrm{j})}, \ldots, \theta_{\mathrm{p}}^{(\mathrm{j})}\right)$ a partir de $\theta^{(\mathrm{j}-1)}$ através de sucessivas gerações de valores,

$$
\begin{gathered}
\theta_{1}^{(j)} \sim \pi\left(\theta_{1} / \theta_{2}^{(j-1)}, \ldots, \theta_{p}^{(j-1)}\right) \\
\theta_{2}^{(j)} \sim \pi\left(\theta_{2} / \theta_{1}^{(j)}, \theta_{3}^{(j-1)}, \ldots, \theta_{p}^{(j-1)}\right) \\
\cdot \\
\theta_{p}^{(j)} \sim \pi\left(\theta_{p} / \theta_{1}^{(j)}, \ldots, \theta_{p-1}^{(j)}\right)
\end{gathered}
$$

(iii) Mude o contador j para $\mathrm{j}+1$ e retorne a (ii) até a convergência. 
À medida que o número de iterações aumenta, a cadeia se aproxima de sua condição de equilíbrio. Assim, assume-se que a convergência é atingida em uma iteração cuja distribuição esteja arbitrariamente próxima da distribuição de equilíbrio $\pi(\theta)$.

O algorítmo Gibbs Sampling define uma cadeia de Markov (ver por exemplo, Ross, 1996) pois os acontecimentos na iteração $\mathrm{j}$ dependem da história do processo apenas através dos valores na iteração $\mathrm{j}-1$. 


\section{O ALGORÍTMO METRÓPOLIS HASTINGS}

Considere uma distribuição $\pi$ da qual se deseja gerar uma amostra através de uma cadeia de Markov (considerando que a geração não-iterativa de $\pi$ é complicada ou custosa). Nesse caso, deve-se construir um núcleo de transição $p(\theta, \phi)$ de forma que $\pi$ seja a distribuição de equilíbrio da cadeia (ver por exemplo, Gamermam, 1996).

Uma forma simples de fazer isso é através de cadeias onde o núcleo $\mathrm{p}$ satisfaça a condição de reversibilidade $\pi(\theta) \mathrm{p}(\theta, \phi)=\pi(\phi) \mathrm{p}(\phi, \theta), \forall(\theta, \phi)$, sendo a condição suficiente para que $\pi$ seja a distribuição de equilíbrio da cadeia.

O núcleo $\mathrm{p}(\theta, \phi)$ é constituído de dois elementos: um núcleo de transição $q(\theta, \phi)$ e uma probabilidade $\alpha(\theta, \phi)$ de forma que:

$$
\mathrm{p}(\theta, \phi)=\mathrm{q}(\theta, \phi) \alpha(\theta, \phi), \text { se } \phi \neq \theta
$$

Portanto, o núcleo de transição define uma densidade $\mathrm{p}(\theta, \cdot)$ para todos os valores diferentes de $\theta$. Consequentemente, resta uma probabilidade positiva da cadeia ficar em $\theta$ dada por,

$$
p(\theta, \theta)=1-\int q(\theta, \phi) \alpha(\theta, \phi) \partial \phi
$$

Essas duas formas podem ser agrupadas na expressão geral:

$$
\mathrm{p}(\theta, \mathrm{A})=\int_{\mathrm{A}} \mathrm{q}(\theta, \phi) \alpha(\theta, \phi) \partial \phi+\mathrm{I}(\theta \in \mathrm{A})\left[1-\int \mathrm{q}(\theta, \phi) \alpha(\theta, \phi) \partial \phi\right] .
$$

Logo, o núcleo de transição define uma distribuição mista para o novo estado $\phi$ da cadeia. Para $\phi \neq \theta$, essa distribuição tem densidade e para $\phi=\theta$, essa distribuição atribui uma probabilidade positiva.

A expressão mais citada para a probabilidade de aceitação é: 


$$
\alpha(\theta, \phi)=\min \left\{1, \frac{\pi(\phi) \mathrm{q}(\phi, \theta)}{\pi(\theta) \mathrm{q}(\theta, \phi)}\right\} .
$$

Em termos práticos isso significa que a simulação de uma amostra $\pi$ usando a cadeia de Markov definida pela transição $p(\theta, A)$ pode ser esquematizada da seguinte forma:

(i) Inicialize o contador de iterações da cadeia $\mathrm{j}=1 \mathrm{e}$ arbitre o valor inicial $\theta^{(0)}$;

(ii) Mova a cadeia para um novo valor $\phi$ gerado da densidade $\mathrm{q}\left(\theta^{(\mathrm{j}-1)},\right)$;

(iii) Calcule a probabilidade de aceitação do movimento $\alpha\left(\theta^{(\mathrm{j}-1)}, \phi\right)$. Se o movimento for aceito, $\theta^{(j)}=\phi$. Se não for aceito, $\theta^{(j)}=\theta^{(j-1)}$ e a cadeia não se move.

(iv) Mude o contador de $\mathrm{j}$ para $\mathrm{j}+1$ e retorne a (ii) até a convergência.

A etapa (iii) é realizada após a geração de uma quantidade uniforme $u$ independente de todas as outras variáveis. Se $\mathrm{u} \leq \alpha$, o movimento é aceito e se $\mathrm{u}>\alpha$ o movimento não é permitido. O núcleo de transição q define uma proposta de movimento que pode ou não ser confirmado por $\alpha$.

O sucesso do método depende de taxas de aceitação não muito baixas e de propostas (q) fáceis de simular. O método substitui uma geração dificil de $\pi$ por várias gerações propostas de q.

\section{Metrópolis em Gibbs}

No caso limite em que se sabe gerar valores de todas as distribuições condicionais, obtemse o amostrador de Gibbs. Mais geralmente, em modelos complexos é possível estabelecer conjugação condicional para algumas, mas não para todas as componentes do modelo.

Nesse caso, sugere-se que a geração das componentes $\theta_{i}$ para as quais não se sabe gerar diretamente de $\pi_{\mathrm{i}}$, seja feita através de uma (sub-)cadeia de Metrópolis Hastings dentro do ciclo do amostrador de Gibbs. Esse processo se daria ao longo de $\mathrm{T}$ iterações culminando com a geração de $\theta_{\mathrm{i}}$ dentro de uma iteração do amostrador de Gibbs. O valor de T seria determinado de forma a garantir a convergência da sub-cadeia para a sua distribuição de equilíbrio $\pi_{\mathrm{i}}$. Esse esquema é denominado esquema amostral de Metrópolis em Gibbs. 
Apêndice 3

\section{VERIFICAÇÃO DE CONVERGÊNCIA}

Uma forma simples de verificar convergência inicializa-se na utilização de várias cadeias em paralelo começando de diferentes pontos, evitando assim, que as cadeias se concentrem em regiões em torno de uma moda local.

Gelman e Rubin elaboraram um método baseado em técnicas de análise de variância. Esse método pode ser descrito por:

Considerando $\mathrm{m}$ cadeias paralelas em uma função real $\mathrm{t}(\theta)$, tem-se $\mathrm{m}$ trajetórias $\left\{\mathrm{t}_{\mathrm{i}}^{(1)}, \mathrm{t}_{\mathrm{i}}^{(2)}, \ldots, \mathrm{t}_{\mathrm{i}}^{(\mathrm{n})}\right\}, \mathrm{i}=1, \ldots, \mathrm{m}$ para $\mathrm{t}$. Portanto, podem ser obtidas a variância entre as cadeias $\mathrm{U}$ e a variância dentro das cadeias $\mathrm{W}$, dadas por:

$$
\mathrm{U}=\frac{\mathrm{n}}{\mathrm{m}-1} \sum_{\mathrm{i}=1}^{\mathrm{m}}\left(\overline{\mathrm{t}}_{\mathrm{i}}-\overline{\mathrm{t}}\right)^{2} \text { e } \mathrm{W}=\sum_{\mathrm{i}=1}^{\mathrm{m}} \frac{\mathrm{s}_{\mathrm{i}}^{2}}{\mathrm{~m}},
$$

onde $\mathrm{s}_{\mathrm{i}}^{2}=\sum_{\mathrm{i}=1}^{\mathrm{n}} \frac{\left(\mathrm{t}_{\mathrm{i}}^{\mathrm{j}}-\overline{\mathrm{t}}_{\mathrm{i}}\right)^{2}}{(\mathrm{n}-1)}, \overline{\mathrm{t}}_{\mathrm{i}}$ é a média das observações da cadeia $\mathrm{i}$ e $\overline{\mathrm{t}}$ é a média dessas médias, $\mathrm{i}=$ $1, \ldots, m$.

Sob convergência, todos os (m.n) valores são gerados da densidade a posteriori, a variância de $t$ pode ser estimada de forma não-viciada por, $\hat{\sigma}^{2}=\frac{n-1}{n} W+\frac{1}{n} U$ e a média desejada pode ser estimada por $\hat{\mu}=\overline{\mathrm{t}}$.

A estimativa da distribuição desejada $\mathrm{N}\left(\hat{\mu}, \hat{\sigma}^{2}\right)$ pode ser corrigida permitindo para a amostragem, variabilidade das estimativas $\hat{\mu}$ e $\hat{\sigma}^{2}$.

$\mathrm{O}$ resultado é uma distribuição 't de Student' aproximada para t com centro $\hat{\mu}$, escala $\sqrt{\hat{V}}=\sqrt{\hat{\sigma}^{2}+U / m n}$ e graus de liberdade $d f=2 \hat{V}^{2} / \operatorname{vâr}(\hat{V})$ onde, 


$$
\begin{aligned}
\operatorname{vâr}(\hat{\mathrm{V}}) & =\left(\frac{\mathrm{n}-1}{\mathrm{n}}\right)^{2} \frac{1}{\mathrm{~m}} \operatorname{vâr}\left(\mathrm{s}_{\mathrm{i}}^{2}\right)+\left(\frac{\mathrm{m}+1}{\mathrm{mn}}\right)^{2} \frac{2}{\mathrm{~m}-1} \mathrm{U}^{2} \times \\
& \times 2 \frac{(\mathrm{m}+1)(\mathrm{n}-1)}{\mathrm{mn}^{2}} \frac{\mathrm{n}}{\mathrm{m}}\left[\operatorname{covv}\left(\mathrm{s}_{\mathrm{i}}^{2}, \overline{\mathrm{t}}_{\mathrm{i}}^{2}\right)-2 \overline{\mathrm{t}} \operatorname{côv}\left(\mathrm{s}_{\mathrm{i}}^{2}, \overline{\mathrm{t}}_{\mathrm{i}}\right)\right]
\end{aligned}
$$

com as variâncias e covariâncias estimadas sendo obtidas dos valores das m amostras.

Um indicador de convergência é dado pelo fator de redução potencial de escala estimado $\sqrt{\hat{\mathrm{R}}}=\sqrt{(\hat{\mathrm{V}} / \mathrm{W}) \partial \mathrm{f} /(\partial \mathrm{f}-2)}$. À medida que $\mathrm{n}$ cresce, $\mathrm{R}$ converge para 1 . $\hat{\mathrm{R}}$ é a razão do estimador da variância atual para a variação dentro da cadeia com um fator que avalia a variância adicional da distribuição 't de Student'.

Se o fator de redução de escala $\hat{\mathrm{R}}$ for alto, temos razões para acreditar que lidar com simulações a posteriori pode fornecer inferências sobre a distribuição desejada.

Desde que $\hat{\mathrm{R}}$ esteja próximo de 1 , admitimos que a convergência ocorreu e as amostras das iterações selecionadas são independentes e identicamente distribuídas. Gelman e Rubin (1992) dão detalhes para racionalizar tal processo.

Uma outra proposta de verificação de convergência é a técnica gráfica sugerida por Gelfand e Smith (1990).

Após um número suficientemente grande de iterações $\mathrm{N}$ em $\mathrm{m}$ cadeias paralelas, forma-se uma amostra de $\theta$ sendo possível a construção de um histograma de qualquer uma de suas componentes. $\mathrm{O}$ mesmo procedimento pode ser repetido após $\mathrm{N}+\mathrm{K}$ iterações.

Não havendo diferença perceptível entre os gráficos obtidos após $\mathrm{N}$ e $\mathrm{N}+\mathrm{K}$ iterações, então conclui-se pela convergência das cadeias.

Ainda dentro da verificação gráfica, é possível observar a trajetória de uma única cadeia ao longo das iterações. Se o gráfico após um período inicial apresenta repetidamente o mesmo comportamento qualitativo e quantitativo então pode se concluir pela convergência da cadeia. 
Apêndice 4

\section{ALGUNS PROGRAMAS DESENVOLVIDOS}

Apresentamos a seguir, alguns programas desenvolvidos nos pacotes estatísticos Minitab e S-Plus utilizados para obter amostras da distribuiçãode interesse através do método Gibbs Sampling e Metrópolis Hastings.

1 - Programa desenvolvido no "Minitab" para obtenção das amostras de $\lambda$ e $N$ ' no modelo de Jelinski e Moranda utilizando o algorítmo Gibbs Sampling.

Set $\mathrm{c} 1\left(\mathrm{x}_{\mathrm{n}}\right)$

92132364345505863707177788791929598104105116149156

247249250

end

let $\mathrm{k} 1=3\left(\mathrm{~N}^{(0)}\right)$

let $\mathrm{k} 2=0.01\left(\lambda^{(0)}\right)$

read $\mathrm{c} 10 \mathrm{c} 11$

00

end

$\operatorname{sum} \mathrm{cl} \mathrm{k} 3\left(\sum_{\mathrm{i}=1}^{26} \mathrm{x}_{\mathrm{i}}\right)$

store ' $a$ '

let $\mathrm{k} 4=1 /(20+250 * \mathrm{k} 1+\mathrm{k} 3)$

random $3 \mathrm{c} 2$;

gamma $26.2 \mathrm{k} 4$

let $\mathrm{k} 2=\mathrm{c} 2(2)$

let $\mathrm{k} 5=30 * \operatorname{expo}(-250 * \mathrm{k} 2)$

random $3 \mathrm{c} 3$;

Poisson k5.

Let $\mathrm{k} 1=\mathrm{c} 3(2)$

stack c10 k1 c10

stack c11 k2 c11

end 
2 - Programa desenvolvido no "Minitab" para obtenção das amostras de $\alpha, \beta_{0}$ e $\beta_{1}$ no modelo de Littlewood e Verral utilizando o algorítmo Gibbs Sampling com Metrópolis Hastings.

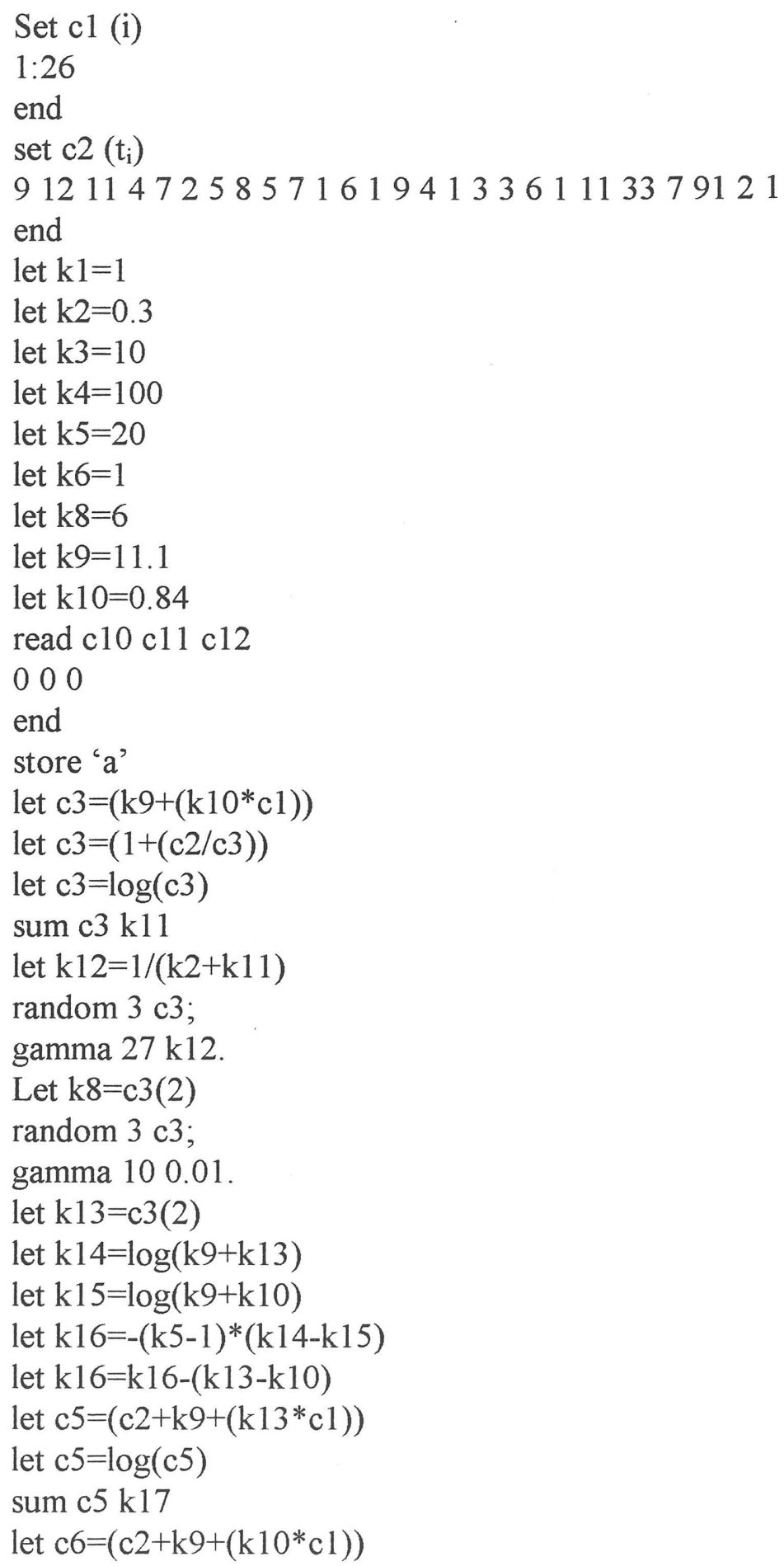




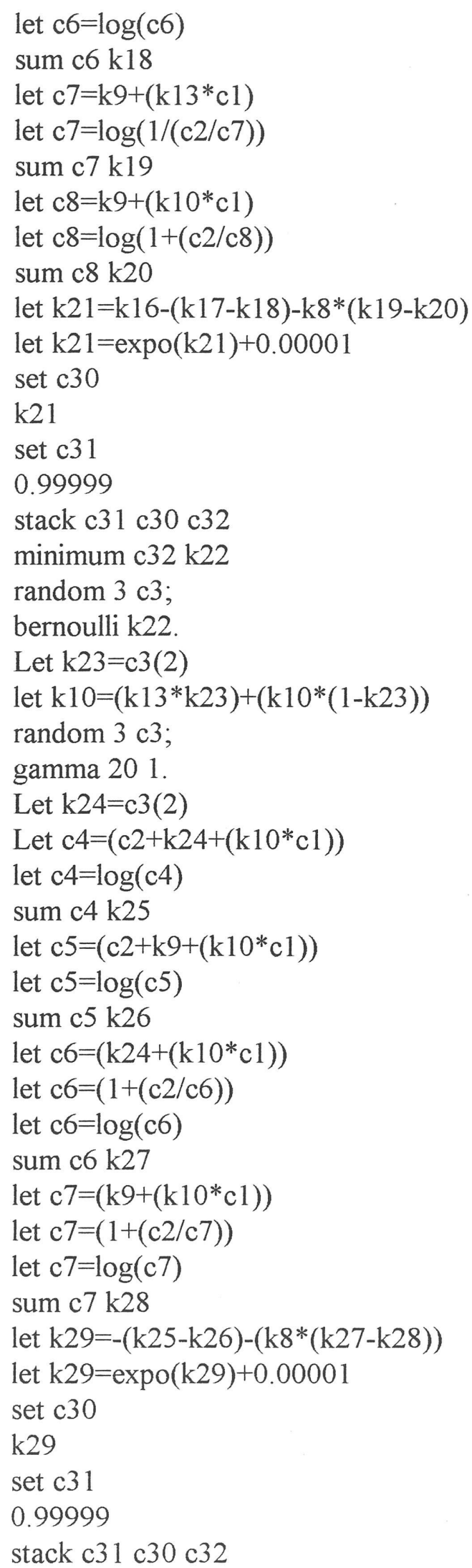


minimum c32 k30

random $3 \mathrm{c} 3$;

bernoulli $\mathrm{k} 30$.

Let $\mathrm{k} 31=\mathrm{c} 3(2)$

let $\mathrm{k} 9=(\mathrm{k} 24 * \mathrm{k} 31)+(\mathrm{k} 9 *(1-\mathrm{k} 31))$

stack c10 k8 c10

stack c11 k9 c11

stack c12 k10 c12

end

3 - Programa desenvolvido no "S-Plus" para obtenção de amostras de $\lambda$ e $N$ ' no modelo de Jelinski e Moranda considerando o segundo caso de prioris hierárquicas descrito no capítulo 3 e utilizando o algorítmo Gibbs Sampling.

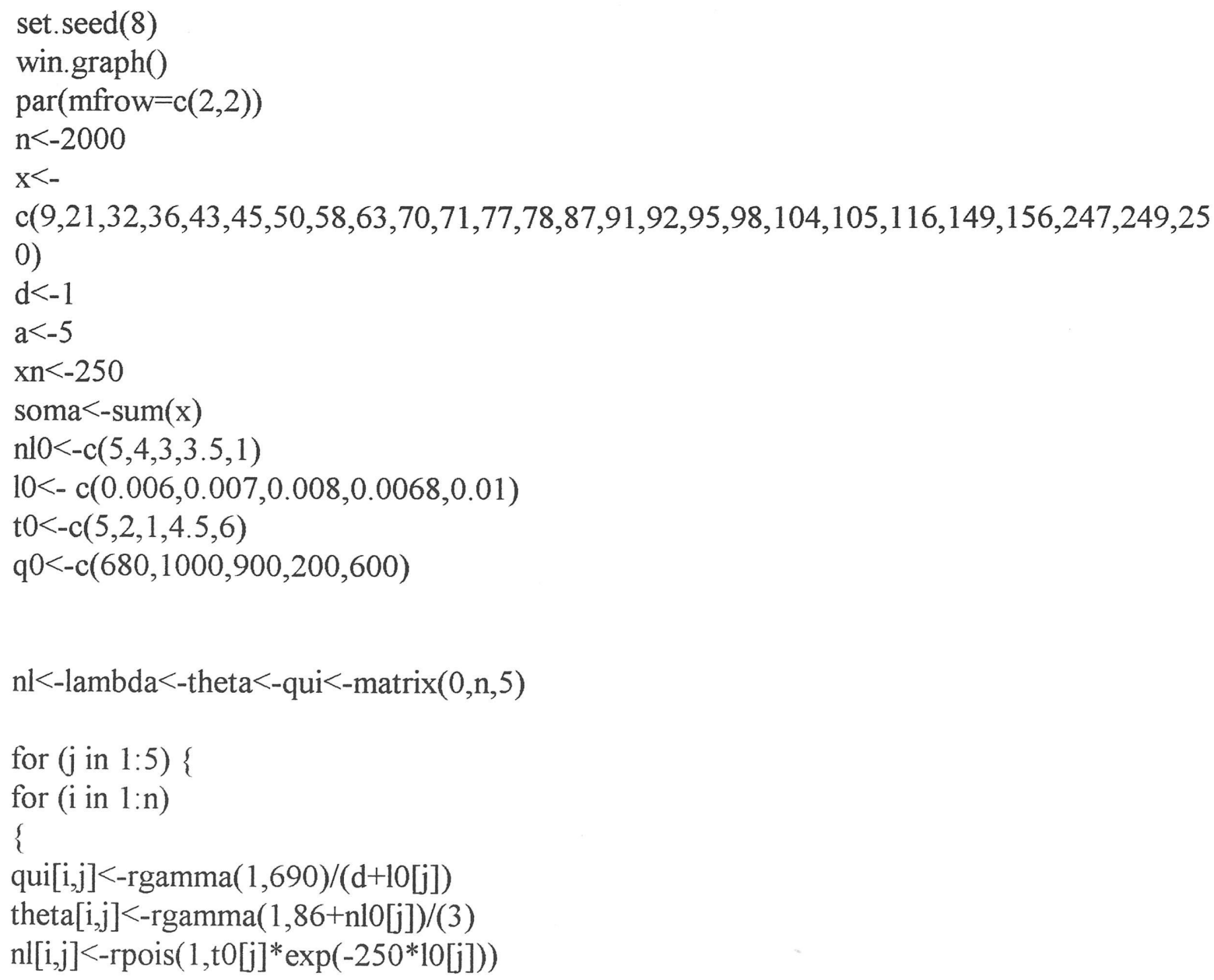




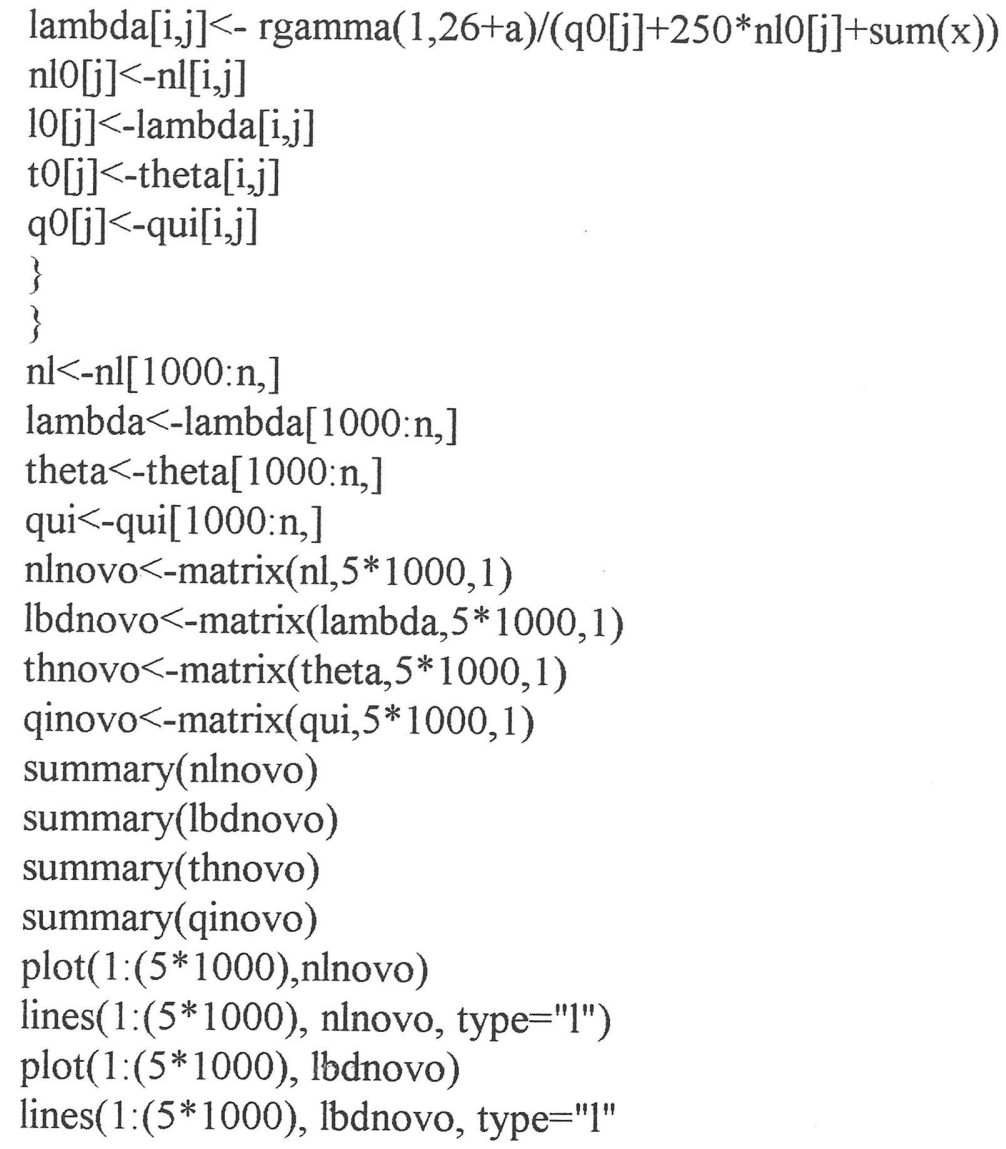




\section{REFERÊNCIAS BIBLIOGRÁFICAS}

ACHCAR, J.A.; SMITH, A.F.M. (1990). Aspects of Reparametrization in Aproximate Bayesian Inference, Essays in Honour of G.A. Barnard. Ed. J. Hodges, pp. 439 - 452. Amsterdam, North Holland.

ACHCAR, J.A. (1995). Use of Approximate Bayesian Inference for Software Reliability. São Carlos, ICMSC-USP, s.p. (Notas do ICMSC-USP, 18).

ACHCAR, J.A. (1996). Bayesian Inference for Software Reliability Models Considering Interfailure Time Data. São Carlos, ICMSC-USP, s.p. (Notas do ICMSC-USP, 30).

AITKIN, M. (1991). Posterior Factor. J.R. Stat. Soc., B, 53, 111-142.

ANSCOMBE, F.J. (1964). Normal Likelihood Functions. Ann. Inst. Stat. Math., 16, 1-19.

BAZARAA, M.S.; SHETTY, C.M. (1979). Nonlinear Programming. New York, John Wiley \& Sons.

BERGER, J.O.; PERICCHI, L.R. (1993). The Intrinsic Bayes Factor for Model Selection and Prediction, Technical Report 93-43 c, dep. of Statistics, Purdue University.

BICKEL, P.J.; DOKSUM, K.A. (1977). Mathematical Statistics. San Francisco, Holden. Day.

BOX, G.E.P.; HILL, W.J. (1967). Discrimination Among Mechanistic Models, Technometrics, $9,57-71$.

BOX, G.E.P.; TIAO, G.G. (1973). Bayesian Inference in Statistical Analysis. New York, Addison-Wesley.

CASELLA, G.; GEORGE, E.I. (1992). Explaining the Gibbs Sampler. The American Statistician, 46, 167-174.

CHANG, H. (1995). Model Determination using Predictive Distributions. Ph.D. Thesis, Department of Statistics, University of Connecticut, Storrs, U.S.A. . 
CHIB, S.; GREENBERG, E. (1995). Understanding the Metrópolis-Hastings Algorithm, The American Statistician, 49, 4, 327-335.

GAMERMAN, D. (1996). Simulação Estocástica via cadeias de Markov, $12^{\circ}$ SINAPESimpósio Nacional de Probabilidade e Estatística, Caxambu, Brasil.

GEISSER, S. ; EDDY, W.F. (1979). A Predictive Approach to Model Selection. J. Am. Stat. Assoc., 74, 153-160.

GELFAND, A.E.; DEY, D.K. (1994). Bayesian Model Choice: Asymptotic and Exact Calculations. J.R. Stat. Soc., B, 3, 501-514.

GELFAND, A.E.; DEY, D.K. e CHANG, H. (1992). Model Determination Using predictive Distribuitions with Implementation via Sampling-Based Methods. In Bayesian Statistics 4 (eds. J.M. Bernardo et al.), pp. 147-167. Oxford: University Press.

GELFAND, A.E.; SMITH, A.F.M. (1990). Sampling-Based Approaches to Calculating Marginal Densities. Journal of the American Statistical Association, 85, 398-409.

GELMAN, A.E.; RUBIN, D. (1992). Inference from Iterative Simulation Using Multiple Sequences, Statistical Science, 7, 457-472.

GOEL, A.L. (1983). A Guidebook for Software Reliability Assessment. Technical Report RADC-TR-83-176, U.S.A.

GOEL, A.L.; OKUMOTO, K. (1978). An Analysis of Recurrent Software Failures on a RealTime Control System, 496-500. Proc. ACM Ann Tech. Conf., Washington, D.C., U.S.A.

GOEL, A.L.; OKUMOTO, K. (1979). Time-Dependent Error Detection Rate Model for Software Reliability and other Performance Measures, IEEE Transactions on Reliability, R-28, 206-211.

HILLS, S.E.; SMITH, A.F.M. (1993). Diagnostic Plots for Improved Parametrization in Bayesian Inference. Biometrika, 80, 61-74.

JEFFREYS, H. (1939). Theory of Probability. Oxford University Press.

JELINSKI, Z.; MORANDA, P.B. (1972). Software Realiability Research. In Statistical Computer Performance Evaluation, ed. W. Freiberger, New York: Academic Press, 465-497. 
KASS, R.E.; SLATE, E.H. (1992). Reparametrization and Diagnostics of Posterior Nonnormality. In Bayesiana Statistics 4, Ed. J.m. Bernardo, J.O. Berger, A.P. Dawid and A.F.M. Smith, pp. 289-306-Oxford University Press.

KLOEK, T.; VAN DIJK, H.K. (1978). Bayesian Estimates of Equation System Parameters: an Application of Integration by Monte Carlo, Econometrika, 46, 1-19.

LAWLESS, J.F. (1982). Statistical Models and Methods for Lifetime Data. New York, John Wiley \& Sons.

LITTLEWOOD, B.; VERRAL, J.L. (1973). A Bayesian Reliability Growth Model for Computer Software, Applied Statistics, 22, 332-346.

MAZZUCHI, T.A.; SOYER, R. (1988). A Bayes Empirical-Bayes Model for Software Reliability, IEEE Transactions on Reliability, R-37: 2, 248-258.

MOOD, A.M.; GRAYBILL, F.A.; BOES, D.C. (1974). Introduction to the Theory of Statistics, $3^{\text {a. }}$ ed., New York, McGraw-Hill.

MORANDA, P.B. (1975). Prediction of Software Reliability and its Applicattions, Proceedings of the Annual Reliability and Maintainability Symposium, 327-332, Washington, D.C., U.S.A.

MUSA, J.D.; OKUMOTO, K. (1984). A Logarithmic Poisson Execution Time Model for Software Reliability Measurement, Proceedings of the $7^{\text {th }}$ International Conference of Software Engineering, Orlando, FL, U.S.A., 230-237.

SCHICK, G.J.; WOLVERTON, R.W. (1978). Assessment of Software Reliability, Proc. Oper. Res., 395-422. Wirzberg-Wien: Physica-Verlag.

SINGPURVALLA, N.D.; WILSON, S.P. (1994). Software Reliability Modeling, International Statistical Review, 62, 3, 289-317.

SPROTT, D.A. (1980). Maximum Likelihood in Small Samples: Estimation in the Presence of Nuisance Pararmeters. Biometrika, v. 67, p. 515-23.

SPROTT, D.A. (1973). Normal Likelihoods and Relation to a Large Sample Theory of Estimations. Biometrika, v. 60, p. 457-65.

STONE, M. (1974). Cross-Validatory Choice and Assessment of Statistical Predictions (with Discussion). J.R.Stat. Soc. B, 36, 111-147. 
TIERNEY, L.; KADANE, J.B. (1986). Accurate Approximations for Posteriori Moments and Marginal Densities. Journal of the American Statistical Associations, v. 81, N. 393, p. 82-6.

YANG, T.Y. (1994). Computational Approaches to Bayesian Inference for Software Reliability. PhD Thesis, Department of Statistics, University of Connecticut, Storrs, U.S.A.

ZACKS, S. (1971). The Theory of Statistical Inference. New York, Willey. 